Supporting Information for:

\title{
Addition of Dithiols to Bis-Ynones: Development of a Versatile Platform for the Synthesis of Polyketide Natural Products
}

Helen F. Sneddon, Matthew J. Gaunt and Steven V. Ley

University Chemical Laboratory; Lensfield Road, Cambridge, UK CB2 1EW.

svl1000@cam.ac.uk 


\section{Supporting Information}

\section{Materials and methods}

All non-aqueous reactions were carried out in oven-dried glassware under an argon atmosphere, unless otherwise noted. All solvents were reagent grade. Diethyl ether and tetrahydrofuran were freshly distilled from sodium benzophenone ketyl under argon. Dichloromethane was freshly distilled from calcium hydride. Pentan-3-one was distilled before use. All other commercially available reagents were used as received.

Except as indicated otherwise, reactions were magnetically stirred and monitored by thin layer chromatography (TLC) with precoated glass-backed plates (Merck Kieselgel 60 F254) and visualized by UV fluorescence $(\lambda=254 \mathrm{~nm})$ or by staining with acidic ammonium molybdate (IV) or acidic potassium permanganate (VII) and developed with appropriate heating. Flash chromatography was performed with Merck Kieselgel (230400 mesh). Yields refer to chromatographically and spectroscopically pure compounds, unless otherwise stated.

Optical rotations were measured on a Perkin-Elmer Model 343 polarimeter with a sodium lamp and are reported as follows: $[\alpha]_{D}^{25}$, [c $(\mathrm{g} / 100 \mathrm{~mL})$, solvent]. Infrared spectra were recorded with a Perkin Elmer Spectrum One FT-IR and are reported in $\mathrm{cm}^{-1}$ (abs). Proton magnetic resonance spectra $\left({ }^{1} \mathrm{H}\right.$ NMR) were recorded at 400 or $600 \mathrm{MHz}$ on a Bruker DPX400 NMR or a Bruker Advance DRX600 instrument respectively and are reported as follows: chemical shift $\delta$ in ppm, (multiplicity, coupling constant $J$ in $\mathrm{Hz}$, number of protons, assignment). Residual protic solvent was used as the internal reference, taking chloroform as $\delta$ 7.26. Carbon magnetic resonance spectra $\left({ }^{13} \mathrm{C}\right.$ NMR $)$ were recorded at 100 or $150 \mathrm{MHz}$ on a Bruker DPX400 NMR or a Bruker Advance DRX600 instrument respectively. Chemical shifts are quoted in ppm and referenced to the appropriate solvent peak, taking chloroform as $\delta 77.0$. The spectra are assigned as fully as possible using the locants derived from the compound name. High-resolution mass spectra were obtained on a Kratos MS890MS spectrometer using electron impact (EI) or fast atom bombardment $(+\mathrm{FAB})$ techniques, or a Kratos Q-TOF spectrometer using electrospray (+ESI) at the Department of Chemistry, University of Cambridge. Microanalyses were determined in the microanalytical laboratories at the University of Cambridge Chemical Laboratories, Lensfield Road, Cambridge, CB2 1EW.

\section{Preparative Experiments}

\section{1-(tert-Butyl-diphenyl-silanyloxy)-hex-5-yne}

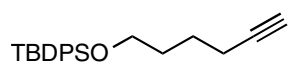

To a solution of 5-hexyn-1-ol (2.00 g, $20.38 \mathrm{mmol})$ and imidazole (3.33 g, $48.91 \mathrm{mmol})$ in tetrahydrofuran $(70 \mathrm{~mL})$ was added tert-butyl-diphenyl-silyl chloride (TBDPSCl) $(5.88$ $\mathrm{g}, 21.40 \mathrm{mmol}$ ). After stirring at ambient temperature for $16 \mathrm{~h}$, the reaction mixture was filtered through a pad of silica and concentrated under reduced pressure. Gradient flash 
chromatography (Petroleum ether/diethyl ether, 100:0 $\rightarrow$ 90:10) afforded the alkyne $(5.22 \mathrm{~g}, 76 \%)$ as a clear colorless oil: $\mathrm{TLC}_{\mathrm{f}}=0.91\left(10 \% \mathrm{Et}_{2} \mathrm{O} / \mathrm{P} . \mathrm{E}\right.$.); IR (thin film) 3309 (m), $2932(\mathrm{~s}), 2858(\mathrm{~s}), 1473(\mathrm{~m}), 1428(\mathrm{~s}), 1100(\mathrm{~s}), 823(\mathrm{~m}), 740(\mathrm{~m}), 702(\mathrm{~s}) \mathrm{cm}^{-1} ;{ }^{1} \mathrm{H}$ NMR (400 MHz, $\left.\mathrm{CDCl}_{3}\right) \delta$ 7.66-7.68 (m, $\left.4 \mathrm{H}, \mathrm{Ph}_{4}\right), 7.36-7.42\left(\mathrm{~m}, 6 \mathrm{H}, \mathrm{Ph} H_{6}\right), 3.68(\mathrm{t}$, $\left.J=5.8 \mathrm{~Hz}, 2 \mathrm{H}, \mathrm{C}_{1}-H_{2}\right), 2.18-2.21\left(\mathrm{~m}, 2 \mathrm{H}, \mathrm{C}_{4}-H_{2}\right), 1.93\left(\mathrm{t}, J=2.5 \mathrm{~Hz}, 1 \mathrm{H}, \mathrm{C}_{6}-H\right), 1.62-$ $1.69\left(\mathrm{~m}, 4 \mathrm{H}, \mathrm{C}_{2}-\mathrm{H}_{2}\right.$ and $\left.\mathrm{C}_{3}-\mathrm{H}_{2}\right), 1.05\left(\mathrm{~s}, 9 \mathrm{H}, \mathrm{OSiPh}_{2} \mathrm{C}\left(\mathrm{CH}_{3}\right)_{3}\right)$; spectra consistent with known data. ${ }^{1}$

\section{7-(tert-Butyl-diphenyl-silanyloxy)-hept-2-ynal}

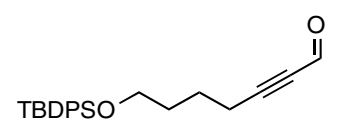

To a solution of 1-(tert-butyl-diphenyl-silanyloxy)-hex-5-yne (3.93 g, $11.67 \mathrm{mmol})$ in tetrahydrofuran $(80 \mathrm{~mL})$ at $-78{ }^{\circ} \mathrm{C}$ was added $n \mathrm{BuLi}(1.6 \mathrm{M}$ in hexane) (4.67 mL, 11.67 mmol) dropwise. After stirring at $-78^{\circ} \mathrm{C}$ for $1 \mathrm{~h}, \mathrm{LiBr}(1.22 \mathrm{~g}, 14.00 \mathrm{mmol})$ was added, followed by DMF $(4.51 \mathrm{~mL}, 58.34 \mathrm{mmol})$ dropwise over $10 \mathrm{~min}$. After stirring at $-78{ }^{\circ} \mathrm{C}$ for a further $30 \mathrm{~min}$. the reaction mixture was allowed to warm to $0{ }^{\circ} \mathrm{C}$ over $16 \mathrm{~h}$, then quenched by the slow addition of saturated aqueous $\mathrm{NH}_{4} \mathrm{Cl}(20 \mathrm{~mL})$. The layers were separated, and the aqueous phase was extracted with diethyl ether $(3 \times 20 \mathrm{~mL})$. The combined organic extracts were washed with $\mathrm{H}_{2} \mathrm{O}(1 \times 20 \mathrm{~mL})$, and brine $(1 \times 20 \mathrm{~mL})$, dried over $\mathrm{MgSO}_{4}$, filtered and concentrated under reduced pressure. Gradient flash chromatography (Petroleum ether/diethyl ether, 100:0 $\rightarrow$ 90:10) afforded the ynal (3.43 $\mathrm{g}, 81 \%$ ) as a clear, colorless oil: TLC $\mathrm{R}_{\mathrm{f}}=0.60$ (10\% $\mathrm{Et}_{2} \mathrm{O} / \mathrm{P} . \mathrm{E}$.); IR (neat) 2934 (m), 2858 (m), 2238 (m), 1688 (s), 1472 (m), 1428 (s), 1280 (m), 1111 (s), 823 (m), 741 (m), 702 (s) $\mathrm{cm}^{-1} ;{ }^{1} \mathrm{H}$ NMR $\left(400 \mathrm{MHz}, \mathrm{CDCl}_{3}\right) \delta 9.17\left(\mathrm{~s}, 1 \mathrm{H}, \mathrm{C}_{1}-H\right)$, 7.67-7.69 (m, $\left.4 \mathrm{H}, \mathrm{PhH}_{4}\right)$, 7.38-7.42 (m, $\left.6 \mathrm{H}, \mathrm{Ph} H_{6}\right), 3.71\left(\mathrm{t}, J=6.0 \mathrm{~Hz}, 2 \mathrm{H}, \mathrm{C}_{7}-H_{2}\right), 2.43\left(\mathrm{t}, J=6.5 \mathrm{~Hz}, 2 \mathrm{H}, \mathrm{C}_{4^{-}}\right.$ $\left.\mathrm{H}_{2}\right), 1.69-1.73\left(\mathrm{~m}, 4 \mathrm{H}, \mathrm{C}_{5}-\mathrm{H}_{2}, \mathrm{C}_{6}-\mathrm{H}_{2}\right), 1.08\left(\mathrm{~s}, 9 \mathrm{H}, \mathrm{OSiPh}_{2} \mathrm{C}\left(\mathrm{CH}_{3}\right)_{3}\right) ;{ }^{13} \mathrm{C} \mathrm{NMR}(100$

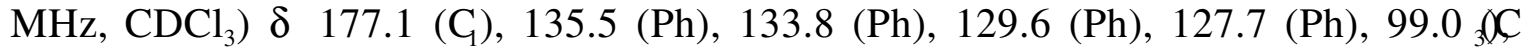
$81.8\left(\mathrm{C}_{2}\right), 63.1\left(\mathrm{C}_{7}\right), 31.5\left(\mathrm{C}_{5}\right), 26.9\left(\mathrm{OSiPh}_{2} \mathrm{C}\left(\mathrm{CH}_{3}\right)_{3}\right), 24.2\left(\mathrm{C}_{6}\right), 19.2\left(\mathrm{OSiPh}_{2} C\left(\mathrm{CH}_{3}\right)_{3}\right)$, $18.9\left(\mathrm{C}_{4}\right)$; high resolution mass spectrum $(+\mathrm{ESI}) \mathrm{m} / \mathrm{z} 364.1872\left[(\mathrm{M}+\mathrm{Na})^{+}\right.$; calcd for $\mathrm{C}_{23} \mathrm{H}_{28} \mathrm{O}_{2} \mathrm{NaSi}$ : 364.1859].

\section{1-(tert-Butyl-diphenyl-silanyloxy)-trideca-5,8-diyn-7-ol}

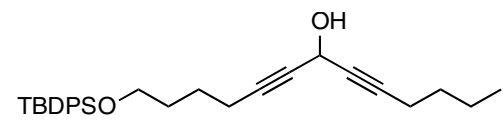

To a solution of hex-1-yne $(0.45 \mathrm{~g}, 5.49 \mathrm{mmol})$ in tetrahydrofuran $(50 \mathrm{~mL})$ at $-78{ }^{\circ} \mathrm{C}$ was added $n \mathrm{BuLi}\left(1.6 \mathrm{M}\right.$ in hexane) $(2.07 \mathrm{~mL}, 5.49 \mathrm{mmol})$ dropwise. After stirring at $-78{ }^{\circ} \mathrm{C}$ for $1 \mathrm{~h}, 7$-(tert-butyl-diphenyl-silanyloxy)-hept-2-ynal $(2.00 \mathrm{~g}, 5.49 \mathrm{mmol})$ was added dropwise over $10 \mathrm{~min}$. After stirring at $-78^{\circ} \mathrm{C}$ for a further $2 \mathrm{~h}$, the reaction mixture was quenched by the slow addition of saturated aqueous $\mathrm{NH}_{4} \mathrm{Cl}(10 \mathrm{~mL})$. The layers were

\footnotetext{
${ }^{1}$ De Oliveira Imbroisi, D.; Simpkins, N. S. J. Chem. Soc. Perkin Trans. 1 1991, 1815.
} 
separated, and the aqueous phase was extracted with ethyl acetate $(2 \times 50 \mathrm{~mL})$. The combined organic extracts were washed with $\mathrm{H}_{2} \mathrm{O}(1 \times 20 \mathrm{~mL})$, and brine $(1 \times 20 \mathrm{~mL})$, dried over $\mathrm{MgSO}_{4}$, filtered and concentrated under reduced pressure. Gradient flash chromatography (Petroleum ether/diethyl ether, 100:0 $\rightarrow$ 90:10) afforded the bis-ynol $(1.07 \mathrm{~g}, 44 \%)$ as a clear, colorless oil: ${ }^{1} \mathrm{H} \mathrm{NMR}\left(400 \mathrm{MHz}, \mathrm{CDCl}_{3}\right) \delta$ 7.65-7.67 (m, $4 \mathrm{H}$, $\mathrm{PhH}_{4}$ ), 7.36-7.42 (m, $\left.6 \mathrm{H}, \mathrm{PhH}_{6}\right), 5.08$ (dqn, $\left.J=7.2,3.0 \mathrm{~Hz}, 1 \mathrm{H}, \mathrm{C}_{7}-H\right), 3.68$ (t, $J=5.9$ $\left.\mathrm{Hz}, 2 \mathrm{H}, \mathrm{C}_{1}-\mathrm{H}_{2}\right), 2.17-2.25\left(\mathrm{~m}, 4 \mathrm{H}, \mathrm{C}_{2}-\mathrm{H}_{2}, \mathrm{C}_{3}-\mathrm{H}_{2}\right), 2.00\left(\mathrm{~d}, J=7.2 \mathrm{~Hz}, 1 \mathrm{H}, \mathrm{C}_{7} \mathrm{OH}\right), 1.60-$ 1.67 (m, $\left.4 \mathrm{H}, \mathrm{C}_{11}-\mathrm{H}_{2}, \mathrm{C}_{12}-\mathrm{H}_{2}\right), 1.47-1.52\left(\mathrm{~m}, 2 \mathrm{H}, \mathrm{C}_{4}-\mathrm{H}_{2}\right), 1.37-1.46\left(\mathrm{~m}, 2 \mathrm{H}, \mathrm{C}_{10}-H_{2}\right), 1.05$ $\left(\mathrm{s}, 9 \mathrm{H}, \mathrm{OSiPh}_{2} \mathrm{C}\left(\mathrm{CH}_{3}\right)_{3}\right), 0.90\left(\mathrm{t}, J=7.2 \mathrm{~Hz}, 3 \mathrm{H}, \mathrm{C}_{13}-\mathrm{H}_{3}\right)$.

\section{1-(tert-Butyl-diphenyl-silanyloxy)-trideca-5,8-diyn-7-one (3a).}

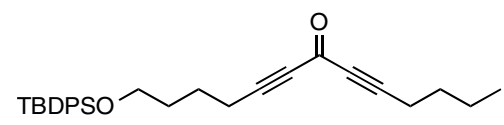

To a solution of 1-(tert-butyl-diphenyl-silanyloxy)-trideca-5,8-diyn-7-ol (1.05g, 2.34 mmol) in dichloromethane $(50 \mathrm{~mL})$ were added $4 \AA$ molecular sieves $(1.17 \mathrm{~g})$, 4methylmorpholine $N$-oxide $(0.41 \mathrm{~g}, 3.52 \mathrm{mmol})$ and tetrapropylammonium perruthenate (TPAP) $(82 \mathrm{mg}, 0.23 \mathrm{mmol})$. The reaction mixture was stirred at ambient temperature for $1 \mathrm{~h}$. The crude reaction mixture was filtered through a plug of silica, washed with diethyl ether $(100 \mathrm{~mL})$ and concentrated under reduced pressure. Gradient flash chromatography (Petroleum ether/diethyl ether, 100:0 $\rightarrow$ 95:5) afforded 3a $(0.75 \mathrm{~g}, 72 \%)$ as a yellow oil: TLC $\mathrm{R}_{\mathrm{f}}=0.58\left(10 \% \mathrm{Et}_{2} \mathrm{O} / \mathrm{P} . \mathrm{E}\right.$.); IR (neat) 2931 (s), 2859 (s), 2206 (s), 1715 (m), 1625 (s), 1472 (m), 1428 (s), 1389 (m), 1361 (m), 1325 (m), 1241 (s), 1187 (m), 1107 (s), 1008 (s), 997 (s), 822 (s), 740 (s), 723 (s), 700 (s) cm ${ }^{-1}$; ${ }^{1} \mathrm{H}$ NMR (400 $\left.\mathrm{MHz} \mathrm{CDCl}_{3}\right) \delta 7.66\left(\mathrm{dd}, J=7.7,1.5 \mathrm{~Hz}, 4 \mathrm{H}, \mathrm{Ph} H_{4}\right), 7.36-7.42\left(\mathrm{~m}, 6 \mathrm{H}, \mathrm{Ph} H_{6}\right), 3.68(\mathrm{t}$, $\left.J=5.7 \mathrm{~Hz}, 2 \mathrm{H}, \mathrm{C}_{1}-H_{2}\right), 2.37-2.41\left(\mathrm{~m}, 4 \mathrm{H}, \mathrm{C}_{2}-H_{2}, \mathrm{C}_{3}-H_{2}\right), 1.61-1.75\left(\mathrm{~m}, 4 \mathrm{H}, \mathrm{C}_{10}-H_{2}, \mathrm{C}_{11^{-}}\right.$ $\left.H_{2}\right), 1.58\left(\mathrm{t}, J=7.5 \mathrm{~Hz}, 2 \mathrm{H}, \mathrm{C}_{4}-H_{2}\right), 1.39-1.48\left(\mathrm{~m}, 2 \mathrm{H}, \mathrm{C}_{12}-H_{2}\right), 1.05(\mathrm{~s}, 9 \mathrm{H}$, $\left.\mathrm{OSiPh}_{2} \mathrm{C}\left(\mathrm{CH}_{3}\right)_{3}\right), 0.92\left(\mathrm{t}, J=7.3 \mathrm{~Hz}, 3 \mathrm{H}, \mathrm{C}_{13}-\mathrm{H}_{3}\right) ;{ }^{13} \mathrm{C} \mathrm{NMR}\left(100 \mathrm{MHz}, \mathrm{CDCl}_{3}\right) \delta 161.8$ $\left(\mathrm{C}_{7}\right), 135.9(\mathrm{Ph}), 134.3(\mathrm{Ph}), 130.0(\mathrm{Ph}), 128.1(\mathrm{Ph}), 95.1\left(\mathrm{C}_{5}\right), 94.8\left(\mathrm{C}_{9}\right), 82.8\left(\mathrm{C}_{6}\right), 82.7$ $\left(\mathrm{C}_{8}\right), 63.5\left(\mathrm{C}_{1}\right), 31.9\left(\mathrm{C}_{2}\right), 29.9,27.3\left(\mathrm{C}_{11}\right), 24.6\left(\mathrm{OSiPh}_{2} \mathrm{C}(\mathrm{CH})_{3}\right), 22.4\left(\mathrm{C}_{12}\right), 19.6\left(\mathrm{C}_{4}\right)$, $19.3\left(\mathrm{C}_{10}\right), 19.2\left(\mathrm{C}_{3}\right), 13.8\left(\mathrm{C}_{13}\right), 1.0\left(\mathrm{OSiPh}_{2} \mathrm{C}(\mathrm{CH})_{3}\right)$; high resolution mass spectrum (+ESI) $\mathrm{m} / z$ 467.2382 [(M+Na $)^{+}$; calcd for $\mathrm{C}_{29} \mathrm{H}_{36} \mathrm{O}_{2} \mathrm{NaSi}$ : 467.2380].

\section{1-(tert-Butyl-diphenyl-silanyloxy)-5-([1,3]dithian-2-yl)-9-([1,3]dithian-2-yl)-trideca-} 7-one (4a).

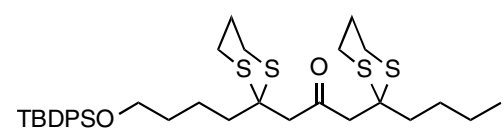

To a solution of 1-(tert-butyl-diphenyl-silanyloxy)-trideca-5,8-diyn-7-one (3a) (50 mg, $0.11 \mathrm{mmol})$ and propane-1,3-dithiol $(27 \mathrm{mg}, 0.25 \mathrm{mmol})$ in tetrahydrofuran $(3 \mathrm{~mL})$ and methanol $(1.5 \mathrm{~mL})$ stirred at $-10{ }^{\circ} \mathrm{C}$ for $30 \mathrm{~min}$. was added sodium hydroxide $(10 \mathrm{mg}$, $0.25 \mathrm{mmol}$ ). The reaction mixture was allowed to warm to ambient temperature over 20 h. The reaction mixture was quenched with saturated aqueous $\mathrm{NH}_{4} \mathrm{Cl}$ solution $(5 \mathrm{~mL})$. 
The layers were separated, and the aqueous phase was extracted with diethyl ether $(3 \times 5$ $\mathrm{mL})$. The combined organic extracts were washed with $\mathrm{H}_{2} \mathrm{O}(1 \times 5 \mathrm{~mL})$, and brine $(1 \times 5$ $\mathrm{mL}$ ), dried over $\mathrm{MgSO}_{4}$, filtered and concentrated under reduced pressure. Gradient flash chromatography (Petroleum ether/diethyl ether, 100:0 $\rightarrow$ 90:10) afforded 4a (67 mg, 90\%) as a clear, pale yellow oil: TLC $\mathrm{R}_{\mathrm{f}}=0.13$ (10\% $\mathrm{Et}_{2} \mathrm{O} / \mathrm{P} . \mathrm{E}$.); IR (neat) $3071(\mathrm{w})$, 2930 (s), 2857 (s), 1717 (s), 1564 (m), 1462 (m), 1427 (s), 1389 (m), 1339 (m), 1276 (m), 1239 (m), 1188 (m), 1107 (s), 998 (s), 939 (m), 908 (m), 869 (m), 822 (s), 740 (s), 701 (s), 687 (s); ${ }^{1} \mathrm{H}$ NMR (400 MHz, $\mathrm{CDCl}_{3}$ ) $\delta$ 7.66-7.67 (m, $\left.4 \mathrm{H}, \mathrm{Ph} H_{4}\right), 7.36-7.45$ (m, $6 \mathrm{H}$, $\left.\mathrm{Ph} H_{6}\right), 3.68\left(\mathrm{t}, J=5.5 \mathrm{~Hz}, 2 \mathrm{H}, \mathrm{C}_{1}-H_{2}\right), 3.14\left(\mathrm{~m}, 4 \mathrm{H}, \mathrm{C}_{6}-H_{2}\right.$ and $\left.\mathrm{C}_{8}-H_{2}\right), 2.78-2.93(\mathrm{~m}, 8$ $\mathrm{H}$, both of $\left.\mathrm{SCH}_{2} \mathrm{CH}_{2} \mathrm{CH}_{2} \mathrm{~S}\right), 2.04-2.10\left(\mathrm{~m}, 4 \mathrm{H}, \mathrm{C}_{4} \mathrm{H}_{2}\right.$ and $\left.\mathrm{C}_{10}-\mathrm{H}_{2}\right), 2.00-2.02(\mathrm{~m}, 2 \mathrm{H}$, one of $\left.\mathrm{SCH}_{2} \mathrm{CH}_{2} \mathrm{CH}_{2} \mathrm{~S}\right), 1.89-1.97\left(\mathrm{~m}, 2 \mathrm{H}\right.$, one of $\left.\mathrm{SCH}_{2} \mathrm{CH}_{2} \mathrm{CH}_{2} \mathrm{~S}\right), 1.56-1.60\left(\mathrm{~m}, 4 \mathrm{H}, \mathrm{C}_{2}-\mathrm{H}_{2}\right.$ and $\left.\mathrm{C}_{3}-H_{2}\right), 1.46-1.52\left(\mathrm{~m}, 2 \mathrm{H}, \mathrm{C}_{11}-H_{2}\right), 1.34\left(\mathrm{qn}, J=7.3 \mathrm{~Hz}, 2 \mathrm{H}, \mathrm{C}_{12}-H_{2}\right), 1.05(\mathrm{~s}, 9 \mathrm{H}$, $\left.\mathrm{OSiPh}_{2} \mathrm{C}\left(\mathrm{CH}_{3}\right)_{3}\right), 0.92\left(\mathrm{t}, J=7.3 \mathrm{~Hz}, 3 \mathrm{H}, \mathrm{C}_{13}-\mathrm{H}_{3}\right) ;{ }^{13} \mathrm{C} \mathrm{NMR}\left(100 \mathrm{MHz}, \mathrm{CDCl}_{3}\right) \delta 202.5$ $\left(\mathrm{C}_{7}\right), 135.6(\mathrm{Ph}), 134.1(\mathrm{Ph}), 129.5(\mathrm{Ph}), 127.6(\mathrm{Ph}), 63.7\left(\mathrm{C}_{1}\right), 51.0\left(\mathrm{C}_{6}\right), 50.6\left(\mathrm{C}_{8}\right), 50.6$

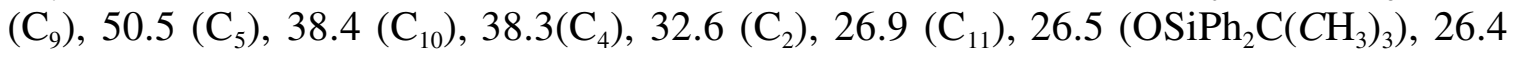
$\left(\mathrm{SCH}_{2} \mathrm{CH}_{2} \mathrm{CH}_{2} \mathrm{~S}\right), 25.0\left(\mathrm{SCH}_{2} \mathrm{CH}_{2} \mathrm{CH}_{2} \mathrm{~S}\right), 22.8\left(\mathrm{C}_{12}\right), 19.2\left(\mathrm{C}_{3}\right), 14.0\left(\mathrm{C}_{13}\right), 1.0$ $\left(\mathrm{OSiPh}_{2} \mathrm{C}\left(\mathrm{CH}_{3}\right)_{3}\right)$; high resolution mass spectrum (+ESI) $\mathrm{m} / \mathrm{z} 683.2517\left[(\mathrm{M}+\mathrm{Na})^{+}\right.$; calcd for $\mathrm{C}_{35} \mathrm{H}_{52} \mathrm{O}_{2} \mathrm{~S}_{4} \mathrm{SiNa}$ : 683.2504].

\section{1-(tert-Butyl-dimethyl-silanyloxy)-but-3-yne}

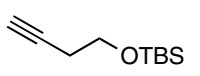

To a solution of 3-butyn-1-ol (9.26 g, $132.1 \mathrm{mmol})$ and imidazole (21.59 g, $317.1 \mathrm{mmol})$ in tetrahydrofuran $(200 \mathrm{~mL})$ was added tert-butyl-dimethyl-silyl chloride (TBSCl) $(23.90$ $\mathrm{g}, 158.5 \mathrm{mmol}$ ). After stirring at ambient temperature for $3 \mathrm{~h}$, the reaction mixture was filtered through a pad of silica and concentrated under reduced pressure. Gradient flash chromatography (Petroleum ether/diethyl ether, 100:0 $\rightarrow$ 90:10) afforded the alkyne $(24.62 \mathrm{~g}, 100 \%)$ as a clear colorless oil: IR (neat) 3315 (m), 2955 (s), 2930 (s), 2858 (s), 1473 (s), 1386 (m), 1362 (m), 1255 (s), 1102 (s), 1060 (s), 1006 (s), 939 (m), 915 (s), 834 (s), $775(\mathrm{~s}) \mathrm{cm}^{-1} ;{ }^{1} \mathrm{H}$ NMR (400 MHz, $\left.\mathrm{CDCl}_{3}\right) \delta 3.75\left(\mathrm{t}, J=7.1 \mathrm{~Hz}, 2 \mathrm{H}, \mathrm{C}_{1}-H_{2}\right), 2.40(\mathrm{td}$, $\left.J=7.1,2.6 \mathrm{~Hz}, 2 \mathrm{H}, \mathrm{C}_{2}-H_{2}\right), 1.96\left(\mathrm{t}, J=2.6 \mathrm{~Hz}, 1 \mathrm{H}, \mathrm{C}_{4}-H\right), 0.90(\mathrm{~s}, 9 \mathrm{H}$, $\left.\mathrm{OSi}\left(\mathrm{CH}_{3}\right)_{2} \mathrm{C}\left(\mathrm{CH}_{3}\right)_{3}\right), 0.08\left(\mathrm{~s}, 6 \mathrm{H}, \mathrm{OSi}\left(\mathrm{CH}_{3}\right)_{2} \mathrm{C}\left(\mathrm{CH}_{3}\right)_{3}\right)$; spectra consistent with known data. $^{2}$

\section{5-(tert-Butyl-dimethyl-silanyloxy)-pent-2-ynal}

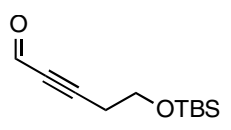

To a solution of 1-(tert-butyl-dimethyl-silanyloxy)-but-3-yne (10.00 g, $52.24 \mathrm{mmol})$ in tetrahydrofuran $(500 \mathrm{~mL})$ stirred at $-78{ }^{\circ} \mathrm{C}$ for $30 \mathrm{~min}$. was added a solution of $n \mathrm{BuLi}$ (1.6 $\mathrm{M}$ in hexane) $(47.46 \mathrm{~mL}, 75.94 \mathrm{mmol})$. The reaction mixture was stirred at $-78{ }^{\circ} \mathrm{C}$ for $1 \mathrm{~h}$. To the reaction mixture was added a solution of $N$-formylmorpholine $(8.74 \mathrm{~g}$,

\footnotetext{
${ }^{2}$ Germain, J.; Deslongchamps, P. J. Org. Chem. 2002, 67, 5269
} 
$7.64 \mathrm{mmol})$ in tetrahydrofuran $(50 \mathrm{~mL})$. The reaction mixture was allowed to warm to ambient temperature over $2 \mathrm{~h}$. The reaction mixture was quenched with saturated solution of aqueous $\mathrm{NH}_{4} \mathrm{Cl}(300 \mathrm{~mL})$. The layers were separated, and the aqueous phase was extracted with diethyl ether $(3 \times 200 \mathrm{~mL})$. The combined organic extracts were washed with $\mathrm{H}_{2} \mathrm{O}(1 \times 200 \mathrm{~mL})$, and brine $(1 \times 200 \mathrm{~mL})$, dried over $\mathrm{MgSO}_{4}$, filtered and concentrated under reduced pressure. Gradient flash chromatography (Petroleum ether/diethyl ether, 100:0 $\rightarrow$ 97:3) afforded the ynal (7.38 g, 64\%) as a yellow oil: TLC $\mathrm{R}_{\mathrm{f}}=0.58\left(10 \% \mathrm{Et}_{2} \mathrm{O} / \mathrm{P} . \mathrm{E}.\right)$; IR (neat) $3256(\mathrm{~m}), 2955(\mathrm{~s}), 2930(\mathrm{~s}), 2885$ (s), $2857(\mathrm{~s})$, 2225 (s), 2098 (s), 1634 (s), $1472(\mathrm{~s}), 1386(\mathrm{~m}), 1362$ (m), 1329 (m), 1253 (s), 1207 (s), $1106(\mathrm{~s}), 1056(\mathrm{~m}), 1006(\mathrm{~s}), 984(\mathrm{~m}), 939(\mathrm{~m}), 909(\mathrm{~s}), 835(\mathrm{~s}), 810(\mathrm{~s}), 776(\mathrm{~s}), 725(\mathrm{~s})$, $684(\mathrm{~s}), 661(\mathrm{~s}) \mathrm{cm}^{-1} ;{ }^{1} \mathrm{H} \mathrm{NMR}\left(400 \mathrm{MHz}, \mathrm{CDCl}_{3}\right) \delta 9.18\left(\mathrm{~s}, 1 \mathrm{H}, \mathrm{C}_{1}-H\right), 3.80(\mathrm{t}, J=6.7$ $\left.\mathrm{Hz}, 2 \mathrm{H}, \mathrm{C}_{5}-\mathrm{H}_{2}\right), 2.63\left(\mathrm{t}, J=6.7 \mathrm{~Hz}, 2 \mathrm{H}, \mathrm{C}_{4}-\mathrm{H}_{2}\right), 0.90\left(\mathrm{~s}, 9 \mathrm{H}, \mathrm{OSi}\left(\mathrm{CH}_{3}\right)_{2} \mathrm{C}\left(\mathrm{CH}_{3}\right)_{3}\right), 0.09$ (s, $\left.6 \mathrm{H}, \mathrm{OSi}\left(\mathrm{CH}_{3}\right)_{2} \mathrm{C}\left(\mathrm{CH}_{3}\right)_{3}\right) ;{ }^{13} \mathrm{C}$ NMR $\left(100 \mathrm{MHz}, \mathrm{CDCl}_{3}\right) \delta 176.9\left(\mathrm{C}_{1}\right), 96.1\left(\mathrm{C}_{3}\right), 82.3$ $\left(\mathrm{C}_{2}\right), 60.6\left(\mathrm{C}_{5}\right), 25.8\left(\mathrm{OSi}\left(\mathrm{CH}_{3}\right)_{2} \mathrm{C}\left(\mathrm{CH}_{3}\right)_{3}\right), 23.5\left(\mathrm{C}_{4}\right), 18.3\left(\mathrm{OSi}_{(}\left(\mathrm{CH}_{3}\right)_{2} \mathrm{C}\left(\mathrm{CH}_{3}\right)_{3}\right),-5.4$ $\left(\mathrm{OSi}\left(\mathrm{CH}_{3}\right)_{2} \mathrm{C}\left(\mathrm{CH}_{3}\right)_{3}\right)$; high resolution mass spectrum (+ESI) $\mathrm{m} / \mathrm{z} 235.1130\left[(\mathrm{M}+\mathrm{Na})^{+}\right.$; calcd for $\left.\mathrm{C}_{11} \mathrm{H}_{20} \mathrm{O}_{2} \mathrm{Na}: 235.1130\right]$.

\section{1-(tert-Butyl-dimethyl-silanyloxy)-8-(tetrahydro-pyran-2-yloxy)-octa-3,6-diyn-5-ol}

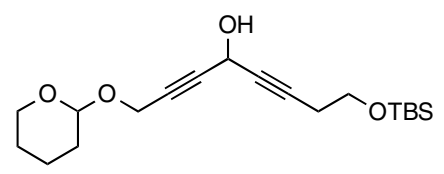

To a solution of 2-prop-2-ynyloxy-tetrahydro-pyran $(0.66 \mathrm{~g}, 4.71 \mathrm{mmol})$ in tetrahydrofuran $(50 \mathrm{~mL})$ stirred at $-78^{\circ} \mathrm{C}$ for $30 \mathrm{~min}$. was added a solution of $n \mathrm{BuLi}(1.6$ $\mathrm{M}$ in hexane) $(2.21 \mathrm{~mL}, 3.53 \mathrm{mmol})$. The reaction mixture was stirred at $-78^{\circ} \mathrm{C}$ for $1 \mathrm{~h}$. The reaction mixture was treated with a solution of 5-(tert-butyl-dimethyl-silanyloxy)pent-2-ynal $(0.50 \mathrm{~g}, 2.35 \mathrm{mmol})$ in tetrahydrofuran $(10 \mathrm{~mL})$. The reaction mixture was stirred at $-78{ }^{\circ} \mathrm{C}$ for $2 \mathrm{~h}$. The reaction mixture was quenched with saturated solution of aqueous $\mathrm{NH}_{4} \mathrm{Cl}(50 \mathrm{~mL})$. The layers were separated, and the aqueous phase was extracted with diethyl ether $(3 \times 50 \mathrm{~mL})$. The combined organic extracts were washed with $\mathrm{H}_{2} \mathrm{O}(1 \times 50 \mathrm{~mL})$, and brine $(1 \times 50 \mathrm{~mL})$, dried over $\mathrm{MgSO}_{4}$, filtered and concentrated under reduced pressure. Gradient flash chromatography (Petroleum ether/diethyl ether, 100:0 $\rightarrow$ 90:10 $\rightarrow$ 80:20 $\rightarrow$ 70:30) afforded the bis-ynol (0.66 g, $80 \%$ ) as a clear yellow oil: TLC $\mathrm{R}_{\mathrm{f}}=0.06$ (10\% $\mathrm{Et}_{2} \mathrm{O} / \mathrm{P} . \mathrm{E}$.); IR (neat) 3397 (br, m), 2929 (s), 2856 (s), 1472 (s), 1442 (s), 1389 (s), 1361 (s), 1299 (s), 1254 (s), 1202 (s), 1184 (s), 1152 (s), 1103 (s), 1079 (s), 1055 (s), 1023 (s), 945 (s), 903 (s), 834 (s), 812 (s), 776 (s), 731 (s), 663 (s) cm $\mathrm{cm}^{-1}{ }^{1} \mathrm{H}$ NMR $\left(400 \mathrm{MHz}, \mathrm{CDCl}_{3}\right) \delta 5.13\left(\mathrm{~d}, J=6.8 \mathrm{~Hz}, 1 \mathrm{H}, \mathrm{C}_{5}-H\right), 4.80$ $(\mathrm{t}, J=3.4 \mathrm{~Hz}, 1 \mathrm{H}, \mathrm{THP}), 4.34\left(\mathrm{dd}, J=15.8,1.8 \mathrm{~Hz}, 1 \mathrm{H}\right.$, one of $\left.\mathrm{C}_{8}-H_{2}\right), 4.26(\mathrm{dd}, J=$ $15.8,1.9 \mathrm{~Hz}, 1 \mathrm{H}$, one of $\left.\mathrm{C}_{8}-H_{2}\right), 3.80-3.86(\mathrm{~m}, 1 \mathrm{H}, \mathrm{THP}), 3.73\left(\mathrm{t}, J=7.1 \mathrm{~Hz}, 2 \mathrm{H}, \mathrm{C}_{1^{-}}\right.$ $\left.\mathrm{H}_{2}\right), 3.51-3.56(\mathrm{~m}, 1 \mathrm{H}, \mathrm{THP}), 2.44\left(\mathrm{dt}, J=7.1,2.1 \mathrm{~Hz}, 2 \mathrm{H}, \mathrm{C}_{2}-H_{2}\right), 1.69-1.84(\mathrm{~m}, 2 \mathrm{H}$, THP), 1.51-1.65 (m, $4 \mathrm{H}$, THP), $0.89\left(\mathrm{~s}, 9 \mathrm{H}, \mathrm{OSi}\left(\mathrm{CH}_{3}\right)_{2} \mathrm{C}\left(\mathrm{CH}_{3}\right)_{3}\right), 0.07(\mathrm{~s}, 6 \mathrm{H}$ $\left.\mathrm{OSi}\left(\mathrm{CH}_{3}\right)_{2} \mathrm{C}\left(\mathrm{CH}_{3}\right)_{3}\right) ;{ }^{13} \mathrm{C} \mathrm{NMR}\left(100 \mathrm{MHz}, \mathrm{CDCl}_{3}\right) \delta 96.9$ (OTHP), $83.4\left(\mathrm{C}_{7}\right), 82.6\left(\mathrm{C}_{3}\right)$, $80.3\left(\mathrm{C}_{6}\right), 78.4\left(\mathrm{C}_{4}\right), 61.9(\mathrm{OTHP}), 61.5\left(\mathrm{C}_{1}\right), 54.2\left(\mathrm{C}_{8}\right), 52.3\left(\mathrm{C}_{5}\right), 30.2(\mathrm{OTHP}), 25.9$ $\left(\mathrm{OSi}\left(\mathrm{CH}_{3}\right)_{2} \mathrm{C}\left(\mathrm{CH}_{3}\right)_{3}\right), 25.3(\mathrm{OTHP}), 23.1\left(\mathrm{C}_{2}\right), 18.9(\mathrm{OTHP}), 18.3\left(\mathrm{OSi}\left(\mathrm{CH}_{3}\right)_{2} \mathrm{C}\left(\mathrm{CH}_{3}\right)_{3}\right)$, - 
$5.3\left(\mathrm{OSi}\left(\mathrm{CH}_{3}\right)_{2} \mathrm{C}\left(\mathrm{CH}_{3}\right)_{3}\right)$; high resolution mass spectrum (+ESI) $\mathrm{m} / z 375.1968\left[(\mathrm{M}+\mathrm{Na})^{+}\right.$; calcd for $\mathrm{C}_{19} \mathrm{H}_{32} \mathrm{O}_{4} \mathrm{NaSi}$ : 375.1968].

1-(tert-Butyl-dimethyl-silanyloxy)-8-(tetrahydro-pyran-2-yloxy)-octa-3,6-diyn-5-one (3b).

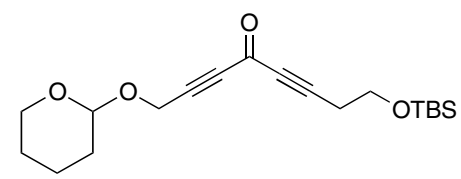

To a solution of 1-(tert-butyl-dimethyl-silanyloxy)-8-(tetrahydro-pyran-2-yloxy)-octa3,6-diyn-5-ol $(0.50 \mathrm{~g}, 1.42 \mathrm{mmol})$ in dichloromethane $(30 \mathrm{~mL})$ were added $4 \AA$ molecular sieves $(1 \mathrm{~g})$, 4-methylmorpholine $N$-oxide $(0.25 \mathrm{~g}, 2.13 \mathrm{mmol})$ and TPAP $(50 \mathrm{mg}, 0.14$ mmol). The reaction mixture was stirred at ambient temperature for $1 \mathrm{~h}$. The crude reaction mixture was filtered through a plug of silica, washed with diethyl ether $(100 \mathrm{~mL})$ and concentrated under reduced pressure. Gradient flash chromatography (Petroleum ether/diethyl ether, 100:0 $\rightarrow$ 90:10) afforded $\mathbf{3 b}(0.16 \mathrm{~g}, 64 \%)$ as a clear yellow oil: TLC $\mathrm{R}_{\mathrm{f}}=0.30$ (20\% $\left.\mathrm{Et}_{2} \mathrm{O} / \mathrm{P} . \mathrm{E}.\right)$; IR (neat) 2930 (s), 2857 (s), 2215 (s), 1631 (s), $1472(\mathrm{~m})$, $1442(\mathrm{~m}), 1388(\mathrm{~m}), 1361(\mathrm{~m}), 1329(\mathrm{~m}), 1236(\mathrm{~s}), 1202(\mathrm{~s}), 1184(\mathrm{~m}), 1112(\mathrm{~s}), 1078(\mathrm{~s})$, 1057 (s), 1030 (s), 958 (s), 941 (s), $904(\mathrm{~s}), 871(\mathrm{~s}), 836(\mathrm{~s}), 814(\mathrm{~s}), 777$ (s), $730(\mathrm{~s}) \mathrm{cm}^{-1}$; ${ }^{1} \mathrm{H}$ NMR (400 MHz, $\left.\mathrm{CDCl}_{3}\right) \delta 4.80$ (t, $\left.J=3.3 \mathrm{~Hz}, 1 \mathrm{H}, \mathrm{OTHP}\right), 4.43$ (s, $\left.2 \mathrm{H}, \mathrm{C}_{8}-H_{2}\right), 3.83$ (m, $1 \mathrm{H}$, OTHP), $3.80\left(\mathrm{t}, J=6.7 \mathrm{~Hz}, 2 \mathrm{H}, \mathrm{C}_{1}-H_{2}\right), 3.54(\mathrm{~m}, 1 \mathrm{H}, \mathrm{OTHP}), 2.61(\mathrm{t}, J=6.7$ $\left.\mathrm{Hz}, 2 \mathrm{H}, \mathrm{C}_{2}-H_{2}\right), 1.78(\mathrm{~m}, 2 \mathrm{H}$, OTHP), $1.58(\mathrm{~m}, 4 \mathrm{H}$, OTHP), $0.90(\mathrm{~s}, 9 \mathrm{H}$, $\left.\mathrm{OSi}\left(\mathrm{CH}_{3}\right)_{2} \mathrm{C}\left(\mathrm{CH}_{3}\right)_{3}\right), 0.09$ (s, $\left.6 \mathrm{H}, \mathrm{OSi}\left(\mathrm{CH}_{3}\right)_{2} \mathrm{C}\left(\mathrm{CH}_{3}\right)_{3}\right) ;{ }^{13} \mathrm{C}$ NMR $\left(100 \mathrm{MHz}, \mathrm{CDCl}_{3}\right)$ $\delta 160.4\left(\mathrm{C}_{5}\right), 97.3$ (THP), $93.0\left(\mathrm{C}_{3}\right), 88.2\left(\mathrm{C}_{6}\right), 85.7\left(\mathrm{C}_{7}\right), 82.5\left(\mathrm{C}_{4}\right), 62.0$ (THP), 60.5 $\left(\mathrm{C}_{1}\right), 53.8\left(\mathrm{C}_{8}\right), 30.1$ (THP), $25.8\left(\mathrm{OSi}\left(\mathrm{CH}_{3}\right)_{2} \mathrm{C}\left(\mathrm{CH}_{3}\right)_{3}\right), 25.2$ (THP), $23.6\left(\mathrm{C}_{2}\right), 18.8$ $\left(\mathrm{OSi}\left(\mathrm{CH}_{3}\right)_{2} \mathrm{C}\left(\mathrm{CH}_{3}\right)_{3}\right)$, 18.3 (THP), -5.4 $\left(\mathrm{OSi}\left(\mathrm{CH}_{3}\right)_{2} \mathrm{C}\left(\mathrm{CH}_{3}\right)_{3}\right)$; high resolution mass spectrum (+ESI) $\mathrm{m} / \mathrm{z} 373.1811\left[(\mathrm{M}+\mathrm{Na})^{+}\right.$; calcd for $\mathrm{C}_{19} \mathrm{H}_{30} \mathrm{O}_{4} \mathrm{NaSi}$ : 373.1811]; elemental analysis $\mathrm{C}, 64.84 \% ; \mathrm{H}, 8.59 \%$.

1-(tert-Butyl-dimethyl-silanyloxy)-3-([1,3]dithian-2-yl)-7-([1,3]dithian-2-yl)-8(tetrahydropyran-2-yloxy)-octan-5-one (4b).

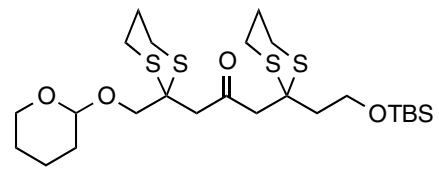

To a solution of 1-(tert-butyl-dimethyl-silanyloxy)-8-(tetrahydro-pyran-2-yloxy)-octa3,6-diyn-5-one (3b) (100 mg, $0.29 \mathrm{mmol})$ and propane-1,3-dithiol (68 mg, $0.63 \mathrm{mmol})$ in dichloromethane $(1 \mathrm{~mL})$ and methanol $(4 \mathrm{~mL})$ stirred at $-10{ }^{\circ} \mathrm{C}$ for $30 \mathrm{~min}$. was added sodium methoxide $(34 \mathrm{mg}, 0.63 \mathrm{mmol}$ ). The reaction mixture was allowed to warm to ambient temperature over $20 \mathrm{~h}$. The reaction mixture was quenched with saturated aqueous $\mathrm{NH}_{4} \mathrm{Cl}$ solution $(10 \mathrm{~mL})$. The layers were separated, and the aqueous phase was extracted with diethyl ether $(3 \times 10 \mathrm{~mL})$. The combined organic extracts were washed with $\mathrm{H}_{2} \mathrm{O}(1 \times 10 \mathrm{~mL})$, and brine $(1 \times 10 \mathrm{~mL})$, dried over $\mathrm{MgSO}_{4}$, filtered and 
concentrated under reduced pressure. Gradient flash chromatography (Petroleum ether/diethyl ether, 100:0 $\rightarrow$ 95:5 $\rightarrow$ 90:10 $\rightarrow$ 80:20) afforded $\mathbf{4 b}(150 \mathrm{mg}, 89 \%)$ as a yellow oil: $\mathrm{TLC} \mathrm{R}_{\mathrm{f}}=0.50$ (50\% Et $\mathrm{Et}_{2} \mathrm{O}$ P.E.); IR (neat) 2929 (s), 2855 (m), 2248 (w), 1715 (m), $1471(\mathrm{~m}), 1424(\mathrm{~m}), 1387(\mathrm{~m}), 1349(\mathrm{~m}), 1277(\mathrm{~m}), 1254(\mathrm{~m}), 1201(\mathrm{~m}), 1184(\mathrm{~m})$, 1120 (s), 1076 (s), 1032 (s), 1004 (m), 976 (s), 907 (s), 869 (s), 835 (s), 814 (s), 776 (s), 728 (s), $661(\mathrm{~s}) \mathrm{cm}^{-1} ;{ }^{1} \mathrm{H}$ NMR $\left(400 \mathrm{MHz}, \mathrm{CDCl}_{3}\right) \delta 4.72$ (t, $J=3.2 \mathrm{~Hz}, 1 \mathrm{H}$, OTHP), $4.17\left(\mathrm{~d}, J=10.5 \mathrm{~Hz}, 1 \mathrm{H}\right.$, one of $\left.\mathrm{C}_{8}-H_{2}\right), 3.91(\mathrm{~m}, 1 \mathrm{H}, \mathrm{OTHP}), 3.88(\mathrm{~d}, J=10.5 \mathrm{~Hz}, 1 \mathrm{H}$, one of $\left.\mathrm{C}_{8}-H_{2}\right), 3.86\left(\mathrm{t}, J=7.0 \mathrm{~Hz}, 2 \mathrm{H}, \mathrm{C}_{1}-H_{2}\right), 3.51(\mathrm{~m}, 1 \mathrm{H}, \mathrm{OTHP}), 3.19$ (s, $2 \mathrm{H}, \mathrm{C}_{4}-H_{2}$ ), $3.11\left(\mathrm{~d}, J=3.5 \mathrm{~Hz}, 2 \mathrm{H}, \mathrm{C}_{6}-\mathrm{H}_{2}\right), 2.97\left(\mathrm{~m}, 2 \mathrm{H}, \mathrm{SCH}_{2} \mathrm{CH}_{2} \mathrm{CH}_{2} \mathrm{~S}\right), 2.86(\mathrm{~m}, 4 \mathrm{H}$, $\left.\mathrm{SCH}_{2} \mathrm{CH}_{2} \mathrm{CH}_{2} \mathrm{~S}\right), 2.76\left(\mathrm{~m}, 2 \mathrm{H}, \mathrm{SCH}_{2} \mathrm{CH}_{2} \mathrm{CH}_{2} \mathrm{~S}\right), 2.36\left(\mathrm{t}, J=7.0 \mathrm{~Hz}, 2 \mathrm{H}, \mathrm{C}_{2}-\mathrm{H}_{2}\right), 1.97(\mathrm{~m}$, $\left.4 \mathrm{H}, \mathrm{SCH}_{2} \mathrm{CH}_{2} \mathrm{CH}_{2} \mathrm{~S}\right), 1.81(\mathrm{~m}, 1 \mathrm{H}, \mathrm{OTHP}), 1.61$ (m, $5 \mathrm{H}$, OTHP), 0.88 (s, 9H, $\left.\mathrm{OSi}\left(\mathrm{CH}_{3}\right)_{2} \mathrm{C}\left(\mathrm{CH}_{3}\right)_{3}\right), 0.06$ (s, 6H, OSi $\left.\left(\mathrm{CH}_{3}\right)_{2} \mathrm{C}\left(\mathrm{CH}_{3}\right)_{3}\right) ;{ }^{13} \mathrm{C}$ NMR $\left(100 \mathrm{MHz}, \mathrm{CDCl}_{3}\right)$ $\delta 202.1\left(\mathrm{C}_{5}\right), 98.8$ (OTHP), $70.3\left(\mathrm{C}_{8}\right), 62.0$ (OTHP), $59.7\left(\mathrm{C}_{1}\right), 51.9$ (OTHP), $50.9\left(\mathrm{C}_{4}\right)$, $50.1\left(\mathrm{C}_{6}\right), 48.8$ (OTHP), $40.7\left(\mathrm{C}_{2}\right), 30.3$ (OTHP), $26.4\left(\mathrm{SCH}_{2} \mathrm{CH}_{2} \mathrm{CH}_{2} \mathrm{~S}\right), 26.3$

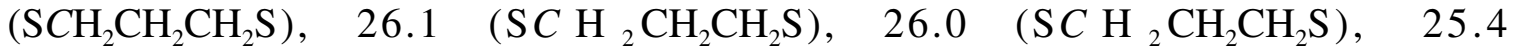
$\left(\mathrm{OSi}\left(\mathrm{CH}_{3}\right)_{2} \mathrm{C}\left(\mathrm{CH}_{3}\right)_{3}\right), 24.9\left(\mathrm{SCH}_{2} \mathrm{CH}_{2} \mathrm{CH}_{2} \mathrm{~S}\right), 24.8\left(\mathrm{SCH}_{2} \mathrm{CH}_{2} \mathrm{CH}_{2} \mathrm{~S}\right), 24.8$ (OTHP), 19.1 (OTHP), $18.3\left(\mathrm{OSi}\left(\mathrm{CH}_{3}\right)_{2} \mathrm{C}\left(\mathrm{CH}_{3}\right)_{3}\right),-5.3\left(\mathrm{OSi}\left(\mathrm{CH}_{3}\right)_{2} \mathrm{C}\left(\mathrm{CH}_{3}\right)_{3}\right)$; high resolution mass spectrum (+ESI) $\mathrm{m} / \mathrm{z} 589.1946\left[(\mathrm{M}+\mathrm{Na})^{+}\right.$; calcd for $\mathrm{C}_{25} \mathrm{H}_{46} \mathrm{O}_{4} \mathrm{~S}_{4} \mathrm{SiNa}$ : 589.1946].

\section{1-(tert-Butyl-dimethyl-silanyloxy)-7-phenyl-hepta-3,6-diyn-5-ol}

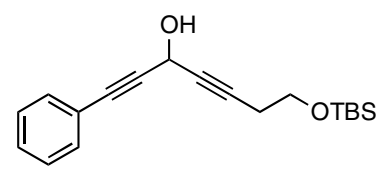

To a solution of ethynyl benzene $(0.48 \mathrm{~g}, 4.71 \mathrm{mmol})$ in tetrahydrofuran $(50 \mathrm{~mL})$ stirred at $-78{ }^{\circ} \mathrm{C}$ for $30 \mathrm{~min}$. was added a solution of $n \mathrm{BuLi}(1.6 \mathrm{M}$ in hexane) $(2.21 \mathrm{~mL}, 3.53$ mmol). The reaction mixture was stirred at $-78{ }^{\circ} \mathrm{C}$ for $1 \mathrm{~h}$. The reaction mixture was treated with a solution of 5-(tert-butyl-dimethyl-silanyloxy)-pent-2-ynal $(0.50 \mathrm{~g}, 2.35$ $\mathrm{mmol})$ in tetrahydrofuran $(10 \mathrm{~mL})$. The reaction mixture was stirred at $-78{ }^{\circ} \mathrm{C}$ for $2 \mathrm{~h}$, then quenched with aqueous $\mathrm{NH}_{4} \mathrm{Cl}(50 \mathrm{~mL})$. The layers were separated, and the aqueous phase was extracted with diethyl ether $(3 \times 50 \mathrm{~mL})$. The combined organic extracts were washed with $\mathrm{H}_{2} \mathrm{O}(1 \times 50 \mathrm{~mL})$, and brine $(1 \times 50 \mathrm{~mL})$, dried over $\mathrm{MgSO}_{4}$, filtered and concentrated under reduced pressure. Gradient flash chromatography (Petroleum ether/diethyl ether, 100:0 $\rightarrow$ 98:2 $\rightarrow$ 96:4 $\rightarrow$ 94:6 $\rightarrow$ 90:10) afforded the bis-ynol (0.60 g, $81 \%)$ as a clear yellow oil: TLC $\mathrm{R}_{\mathrm{f}}=0.19\left(10 \% \mathrm{Et}_{2} \mathrm{O} / \mathrm{P} . \mathrm{E}\right.$.); IR (neat) $3399(\mathrm{br}, \mathrm{m}), 2953$ (s), 2928 (s), 2856 (s), 2236 (w), 1682 (w), 1599 (w), 1490 (s), 1472 (s), 1444 (s), 1387 (s), $1362(\mathrm{~s}), 1334(\mathrm{~m}), 1301(\mathrm{~s}), 1253(\mathrm{~s}), 1220(\mathrm{~m}), 1136(\mathrm{~s}), 1100(\mathrm{~s}), 1056(\mathrm{~s}), 1030$ (s), 1015 (s), 998 (s), 976 (s), 952 (s), 910 (s), 834 (s), 809 (s), 776 (s), 755 (s), 733 (s), $690(\mathrm{~s}), 665(\mathrm{~s}) \mathrm{cm}^{-1} ;{ }^{1} \mathrm{H}$ NMR $\left(400 \mathrm{MHz}, \mathrm{CDCl}_{3}\right) \delta 7.47\left(\mathrm{~m}, 2 \mathrm{H}, \mathrm{Ph} H_{2}\right), 7.28-7.36(\mathrm{~m}, 3$ $\left.\mathrm{H}, \mathrm{Ph} H_{3}\right), 5.33\left(\mathrm{dt}, J=7.3,2.0 \mathrm{~Hz}, 1 \mathrm{H}, \mathrm{C}_{5}-H\right), 3.76\left(\mathrm{t}, J=7.0 \mathrm{~Hz}, 2 \mathrm{H}, \mathrm{C}_{1}-H_{2}\right), 2.49(\mathrm{dt}, J$ $\left.=7.0,2.0 \mathrm{~Hz}, 2 \mathrm{H}, \mathrm{C}_{2}-H_{2}\right), 2.34\left(\mathrm{~d}, J=7.3 \mathrm{~Hz}, 1 \mathrm{H}, \mathrm{C}_{5} \mathrm{OH}\right), 0.90(\mathrm{~s}, 9 \mathrm{H}$, $\left.\mathrm{OSi}\left(\mathrm{CH}_{3}\right)_{2} \mathrm{C}\left(\mathrm{CH}_{3}\right)_{3}\right), 0.09\left(\mathrm{~s}, 6 \mathrm{H}, \mathrm{OSi}\left(\mathrm{CH}_{3}\right)_{2} \mathrm{C}\left(\mathrm{CH}_{3}\right)_{3}\right) ;{ }^{13} \mathrm{C} \mathrm{NMR}\left(100 \mathrm{MHz}, \mathrm{CDCl}_{3}\right)$ $\delta 131.8(\mathrm{Ph}), 128.7(\mathrm{Ph}), 128.3(\mathrm{Ph}), 122.1(\mathrm{Ph}), 86.5\left(\mathrm{C}_{6}\right), 84.0\left(\mathrm{C}_{7}\right), 82.8\left(\mathrm{C}_{3}\right), 78.5$ $\left(\mathrm{C}_{4}\right), 61.5\left(\mathrm{C}_{1}\right), 52.8\left(\mathrm{C}_{5}\right), 25.9\left(\mathrm{OSi}\left(\mathrm{CH}_{3}\right)_{2} \mathrm{C}\left(\mathrm{CH}_{3}\right)_{3}\right), 23.2\left(\mathrm{C}_{2}\right), 18.3\left(\mathrm{OSi}\left(\mathrm{CH}_{3}\right)_{2} C\left(\mathrm{CH}_{3}\right)_{3}\right)$, 


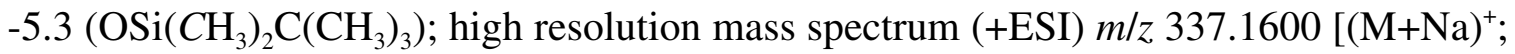
calcd for $\mathrm{C}_{19} \mathrm{H}_{26} \mathrm{O}_{2} \mathrm{NaSi}$ : 337.1600]; elemental analysis $\mathrm{C}, 72.70 \%$; $\mathrm{H}, 8.31 \%$.

1-(tert-Butyl-dimethyl-silanyloxy)-7-phenyl-hepta-3,6-diyn-5-one (3c).

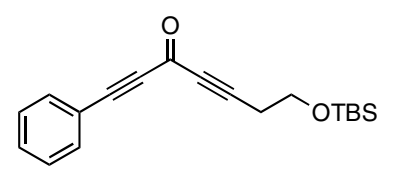

To a solution of 1-(tert-butyl-dimethyl-silanyloxy)-7-phenyl-hepta-3,6-diyn-5-ol (0.20g, $0.64 \mathrm{mmol})$ in dichloromethane $(15 \mathrm{~mL})$ were added $4 \AA$ molecular sieves $(0.5 \mathrm{~g}), 4$ methylmorpholine $N$-oxide $(0.11 \mathrm{~g}, 0.94 \mathrm{mmol})$ and TPAP $(22 \mathrm{mg}, 0.06 \mathrm{mmol})$. The reaction mixture was stirred at ambient temperature for $1 \mathrm{~h}$. The crude reaction mixture was filtered through a plug of silica, washed with diethyl ether $(50 \mathrm{~mL})$ and concentrated under reduced pressure. Gradient flash chromatography (Petroleum ether/diethyl ether, 100:0 $\rightarrow$ 80:20) afforded 3c $(0.16 \mathrm{~g}, 82 \%)$ as a clear yellow oil: TLC $\mathrm{R}_{\mathrm{f}}=0.50(20 \%$ $\mathrm{Et}_{2}$ O/P.E.); IR (neat) 2955 (m), 2929 (m), 2857 (m), 2198 (s), 1622 (s), 1490 (m), 1472 (m), $1444(\mathrm{~m}), 1383(\mathrm{~m}), 1362(\mathrm{~m}), 1329(\mathrm{~m}), 1284(\mathrm{~s}), 1253(\mathrm{~s}), 1183(\mathrm{~s}), 1172(\mathrm{~s}), 1108$ (s), $1056(\mathrm{~m}), 1027(\mathrm{~s}), 1012(\mathrm{~s}), 997(\mathrm{~s}), 909$ (s), 835 (s), 809 (s), 777 (s), 757 (s), 737 (s), $716(\mathrm{~s}), 688(\mathrm{~s}), 660(\mathrm{~s}) \mathrm{cm}^{-1} ;{ }^{1} \mathrm{H}$ NMR $\left(400 \mathrm{MHz}, \mathrm{CDCl}_{3}\right) \delta 7.60(\mathrm{~d}, J=7.4 \mathrm{~Hz}, 2 \mathrm{H}$, $\left.\mathrm{Ph} H_{2}\right), 7.48$ (t, $\left.J=7.4 \mathrm{~Hz}, 1 \mathrm{H}, \mathrm{Ph} H\right), 7.39\left(\mathrm{t}, J=7.4 \mathrm{~Hz}, 2 \mathrm{H}, \mathrm{Ph} H_{2}\right), 3.84(\mathrm{t}, J=6.7 \mathrm{~Hz}$, $\left.2 \mathrm{H}, \mathrm{C}_{1}-H_{2}\right), 2.66\left(\mathrm{t}, J=6.7 \mathrm{~Hz}, 2 \mathrm{H}, \mathrm{C}_{2}-H_{2}\right), 0.91\left(\mathrm{~s}, 9 \mathrm{H}, \mathrm{OSi}\left(\mathrm{CH}_{3}\right)_{2} \mathrm{C}\left(\mathrm{CH}_{3}\right)_{3}\right), 0.11(\mathrm{~s}, 6$ $\left.\mathrm{H}, \mathrm{OSi}\left(\mathrm{CH}_{3}\right)_{2} \mathrm{C}\left(\mathrm{CH}_{3}\right)_{3}\right) ;{ }^{13} \mathrm{C}$ NMR $\left(100 \mathrm{MHz}, \mathrm{CDCl}_{3}\right) \delta 160.9\left(\mathrm{C}_{5}\right), 133.3(\mathrm{Ph}), 131.1$ $(\mathrm{Ph}), 128.6(\mathrm{Ph}), 119.6(\mathrm{Ph}), 92.7\left(\mathrm{C}_{3}\right), 90.7\left(\mathrm{C}_{7}\right), 89.3\left(\mathrm{C}_{6}\right), 82.8\left(\mathrm{C}_{4}\right), 60.6\left(\mathrm{C}_{1}\right), 25.8$ $\left(\mathrm{OSi}\left(\mathrm{CH}_{3}\right)_{2} \mathrm{C}\left(\mathrm{CH}_{3}\right)_{3}\right), 23.6\left(\mathrm{C}_{2}\right), 18.3\left(\mathrm{OSi}\left(\mathrm{CH}_{3}\right)_{2} \mathrm{C}\left(\mathrm{CH}_{3}\right)_{3}\right),-5.3\left(\mathrm{OSi}\left(\mathrm{CH}_{3}\right)_{2} \mathrm{C}\left(\mathrm{CH}_{3}\right)_{3}\right)$; high resolution mass spectrum $(+\mathrm{ESI}) \mathrm{m} / \mathrm{z} 335.1443\left[(\mathrm{M}+\mathrm{Na})^{+}\right.$; calcd for $\mathrm{C}_{19} \mathrm{H}_{24} \mathrm{O}_{2} \mathrm{NaSi}$ : 335.1443]; elemental analysis C, 72.78\%; H, 7.67\%.

1-(tert-Butyl-dimethyl-silanyloxy)-3-([1,3]dithian-2-yl)-7-([1,3]dithian-2-yl)-7phenyl-heptan-5-one (4c).

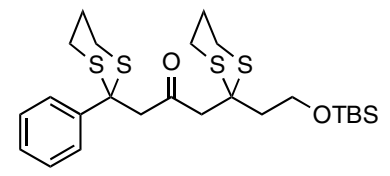

To a solution of 1-(tert-butyl-dimethyl-silanyloxy)-7-phenyl-hepta-3,6-diyn-5-one (3c) $(100 \mathrm{mg}, 0.32 \mathrm{mmol})$ and propane-1,3-dithiol $(76 \mathrm{mg}, 0.70 \mathrm{mmol})$ in dichloromethane $(1$ $\mathrm{mL}$ ) and methanol $\left(4 \mathrm{~mL}\right.$ ) stirred at $-10^{\circ} \mathrm{C}$ for $30 \mathrm{~min}$. was added sodium methoxide (38 $\mathrm{mg}, 0.70 \mathrm{mmol})$. The reaction mixture was allowed to warm to ambient temperature over $20 \mathrm{~h}$. The reaction mixture was quenched with saturated aqueous $\mathrm{NH}_{4} \mathrm{Cl}$ solution $(10$ $\mathrm{mL}$ ). The layers were separated, and the aqueous phase was extracted with diethyl ether $(3 \times 10 \mathrm{~mL})$. The combined organic extracts were washed with $\mathrm{H}_{2} \mathrm{O}(1 \times 10 \mathrm{~mL})$, and brine $(1 \times 10 \mathrm{~mL})$, dried over $\mathrm{MgSO}_{4}$, filtered and concentrated under reduced pressure. Gradient flash chromatography (Petroleum ether/diethyl ether, 100:0 $\rightarrow$ 95:5 $\rightarrow$ 94:6 $\rightarrow$ $93: 7 \rightarrow 92: 8)$ afforded $\mathbf{4 c}(136 \mathrm{mg}, 80 \%)$ as a yellow oil: $\mathrm{R}_{\mathrm{f}}=0.75\left(50 \% \mathrm{Et}_{2} \mathrm{O} / \mathrm{P} . \mathrm{E}\right.$.); IR 
(neat) $2929(\mathrm{~s}), 2856(\mathrm{~s}), 2250(\mathrm{w}), 1710(\mathrm{~s}), 1471(\mathrm{~m}), 1443$ (s), $1423(\mathrm{~s}), 1389(\mathrm{~m})$, 1340 (m), 1277 (s), 1251 (s), 1090 (s), 1035 (s), 1004 (s), 908 (s), 835 (s), 776 (s), 729 (s), $700(\mathrm{~s}), 662$ (s) $\mathrm{cm}^{-1}$; ${ }^{1} \mathrm{H}$ NMR $\left(400 \mathrm{MHz}, \mathrm{CDCl}_{3}\right) \delta 7.95\left(\mathrm{~d}, J=7.5 \mathrm{~Hz}, 2 \mathrm{H}, \mathrm{PhH}_{2}\right.$ ), $7.40\left(\mathrm{t}, J=7.5 \mathrm{~Hz}, 2 \mathrm{H}, \mathrm{Ph} H_{2}\right), 7.29(\mathrm{t}, J=7.5 \mathrm{~Hz}, 1 \mathrm{H}, \mathrm{Ph} H), 3.74(\mathrm{t}, J=7.1 \mathrm{~Hz}, 2 \mathrm{H}$, $\left.\mathrm{C}_{1}-H_{2}\right), 3.24\left(\mathrm{~s}, 2 \mathrm{H}, \mathrm{C}_{6}-H_{2}\right), 2.78\left(\mathrm{~s}, 2 \mathrm{H}, \mathrm{C}_{4}-H_{2}\right), 2.67\left(\mathrm{~m}, 8 \mathrm{H}, \mathrm{SCH}_{2} \mathrm{CH}_{2} \mathrm{CH}_{2} \mathrm{~S}\right), 2.20$ (t, J $\left.=7.1 \mathrm{~Hz}, 2 \mathrm{H}, \mathrm{C}_{2}-\mathrm{H}_{2}\right), 1.95\left(\mathrm{~m}, 2 \mathrm{H} \mathrm{SCH}_{2} \mathrm{CH}_{2} \mathrm{CH}_{2} \mathrm{~S}\right), 1.87\left(\mathrm{~m}, 2 \mathrm{H}, \mathrm{SCH}_{2} \mathrm{CH}_{2} \mathrm{CH}_{2} \mathrm{~S}\right), 0.87$ (s, $\left.9 \mathrm{H}, \mathrm{OSi}\left(\mathrm{CH}_{3}\right)_{2} \mathrm{C}\left(\mathrm{CH}_{3}\right)_{3}\right), 0.03\left(\mathrm{~s}, 6 \mathrm{H}, \mathrm{OSi}\left(\mathrm{CH}_{3}\right)_{2} \mathrm{C}\left(\mathrm{CH}_{3}\right)_{3}\right) ;{ }^{13} \mathrm{C} \mathrm{NMR}(100 \mathrm{MHz}$, $\left.\mathrm{CDCl}_{3}\right) \delta 201.0\left(\mathrm{C}_{5}\right), 140.8(\mathrm{Ph}), 128.7(\mathrm{Ph}), 128.6(\mathrm{Ph}), 127.6(\mathrm{Ph}), 59.5\left(\mathrm{C}_{1}\right), 58.0\left(\mathrm{C}_{6}\right)$, $55.2\left(\mathrm{C}_{7}\right), 51.5\left(\mathrm{C}_{4}\right), 48.6\left(\mathrm{C}_{3}\right), 40.9\left(\mathrm{C}_{2}\right), 27.7\left(\mathrm{SCH}_{2} \mathrm{CH}_{2} \mathrm{CH}_{2} \mathrm{~S}\right), 27.7\left(\mathrm{SCH}_{2} \mathrm{CH}_{2} \mathrm{CH}_{2} \mathrm{~S}\right)$, $26.4\left(\mathrm{SCH}_{2} \mathrm{CH}_{2} \mathrm{CH}_{2} \mathrm{~S}\right), 26.4 \quad\left(\mathrm{SCH}_{2} \mathrm{CH}_{2} \mathrm{CH}_{2} \mathrm{~S}\right), 26.0 \quad\left(\mathrm{OSi}\left(\mathrm{CH}_{3}\right)_{2} \mathrm{C}\left(\mathrm{CH}_{3}\right)_{3}\right), \quad 24.7$

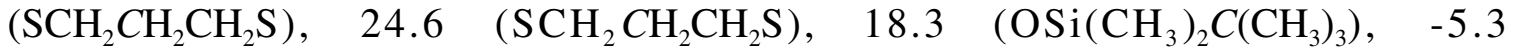
$\left(\mathrm{OSi}\left(\mathrm{CH}_{3}\right)_{2} \mathrm{C}\left(\mathrm{CH}_{3}\right)_{3}\right)$; high resolution mass spectrum (+ESI) $\mathrm{m} / \mathrm{z} 551.1578\left[(\mathrm{M}+\mathrm{Na})^{+}\right.$; calcd for $\mathrm{C}_{25} \mathrm{H}_{40} \mathrm{O}_{2} \mathrm{~S}_{4} \mathrm{SiNa}$ : 551.1578].

\section{1-(tert-Butyl-dimethyl-silanyloxy)-hepta-3,6-diyn-5-ol}

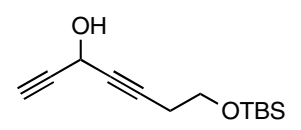

To a solution of 5-(tert-butyl-dimethyl-silanyloxy)-pent-2-ynal (1.00 g, $4.71 \mathrm{mmol})$ in tetrahydrofuran $(10 \mathrm{~mL})$ stirred at $-78{ }^{\circ} \mathrm{C}$ for $30 \mathrm{~min}$. was added a solution of ethynylmagnesium bromide $(0.5 \mathrm{M}$ in tetrahydrofuran) $(18.8 \mathrm{~mL}, 9.42 \mathrm{mmol})$. The reaction mixture was allowed to warm to $-30^{\circ} \mathrm{C}$ and stirred for $2 \mathrm{~h}$. The reaction mixture was quenched with saturated solution of aqueous $\mathrm{NH}_{4} \mathrm{Cl}(10 \mathrm{~mL})$. The layers were separated, and the aqueous phase was extracted with diethyl ether $(3 \times 10 \mathrm{~mL})$. The combined organic extracts were washed with $\mathrm{H}_{2} \mathrm{O}(1 \times 10 \mathrm{~mL})$, and brine $(1 \times 10 \mathrm{~mL})$, dried over $\mathrm{MgSO}_{4}$, filtered and concentrated under reduced pressure. Gradient flash chromatography (Petroleum ether/diethyl ether, 100:0 $\rightarrow$ 95:5 $\rightarrow$ 90:10) afforded the bisynol $(0.83 \mathrm{~g}, 74 \%)$ as a clear yellow oil: TLC $\mathrm{R}_{\mathrm{f}}=0.07$ (5\% Et $\mathrm{Et}_{2} \mathrm{O} / \mathrm{P} . \mathrm{E}$.); IR (neat) 3312 (br, m), 2955 (s), 2930 (s), 2857 (s), 1472 (s), 1386 (s), 1362 (m), 1297 (m), 1254 (s), 1139 (s), 1100 (s), 1057 (s), 1023 (s), 972 (m), 914 (s), 835 (s), 809 (s), 776 (s), 660 (s) $\mathrm{cm}^{-1} ;{ }^{1} \mathrm{H} \mathrm{NMR}\left(600 \mathrm{MHz}, \mathrm{CDCl}_{3}\right) \delta 5.09\left(\mathrm{dd}, J=7.4,2.2 \mathrm{~Hz}, 1 \mathrm{H}, \mathrm{C}_{5}-H\right), 3.74(\mathrm{t}, J=7.0$ $\left.\mathrm{Hz}, 2 \mathrm{H}, \mathrm{C}_{1}-H_{2}\right), 2.54\left(\mathrm{~d}, J=2.2 \mathrm{~Hz}, 1 \mathrm{H}, \mathrm{C}_{7}-H\right), 2.46\left(\mathrm{dt}, J=7.0,2.2 \mathrm{~Hz}, 2 \mathrm{H}, \mathrm{C}_{2}-H_{2}\right)$, $2.17\left(\mathrm{~d}, J=7.4 \mathrm{~Hz}, 1 \mathrm{H}, \mathrm{C}_{5} \mathrm{OH}\right), 0.90\left(\mathrm{~s}, 9 \mathrm{H}, \mathrm{OSi}\left(\mathrm{CH}_{3}\right)_{2} \mathrm{C}\left(\mathrm{CH}_{3}\right)_{3}\right), 0.08(\mathrm{~s}, 6 \mathrm{H}$, $\left.\mathrm{OSi}\left(\mathrm{CH}_{3}\right)_{2} \mathrm{C}\left(\mathrm{CH}_{3}\right)_{3}\right) ;{ }^{13} \mathrm{C} \mathrm{NMR}\left(150 \mathrm{MHz}, \mathrm{CDCl}_{3}\right) \delta 83.0\left(\mathrm{C}_{4}\right), 81.3\left(\mathrm{C}_{3}\right), 78.0\left(\mathrm{C}_{5}\right), 72.3$ $\left(\mathrm{C}_{7}\right), 61.4\left(\mathrm{C}_{1}\right), 52.1\left(\mathrm{C}_{6}\right), 25.9\left(\mathrm{OSi}\left(\mathrm{CH}_{3}\right)_{2} \mathrm{C}\left(\mathrm{CH}_{3}\right)_{3}\right), 23.1\left(\mathrm{C}_{2}\right), 18.3\left(\mathrm{OSi}\left(\mathrm{CH}_{3}\right)_{2} \mathrm{C}\left(\mathrm{CH}_{3}\right)_{3}\right)$, $-5.3\left(\mathrm{OSi}\left(\mathrm{CH}_{3}\right)_{2} \mathrm{C}\left(\mathrm{CH}_{3}\right)_{3}\right)$; high resolution mass spectrum (+ESI) $\mathrm{m} / \mathrm{z} 261.1287\left[(\mathrm{M}+\mathrm{Na})^{+}\right.$; calcd for $\mathrm{C}_{13} \mathrm{H}_{22} \mathrm{O}_{2} \mathrm{SiNa}$ : 261.1287].

\section{1-(tert-Butyl-dimethyl-silanyloxy)-hepta-3,6-diyn-5-one (3d).}

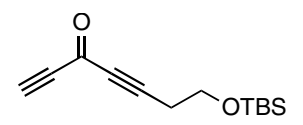

To a solution of 1-(tert-butyl-dimethyl-silanyloxy)-hepta-3,6-diyn-5-ol (1.04 g, 4.35 $\mathrm{mmol})$ in dichloromethane $(50 \mathrm{~mL})$ stirred at $0{ }^{\circ} \mathrm{C}$ for $30 \mathrm{~min}$. was added Dess-Martin 
periodinane $(2.77 \mathrm{~g}, 6.52 \mathrm{mmol})$. The reaction mixture was allowed to warm to ambient temperature and stirred for $2 \mathrm{~h}$. The reaction mixture was quenched with a 1:1 solution of saturated aqueous $\mathrm{Na}_{2} \mathrm{~S}_{2} \mathrm{O}_{3}$ and saturated aqueous $\mathrm{NaHCO}_{3}(50 \mathrm{~mL})$. The layers were separated, and the aqueous phase was extracted with diethyl ether $(3 \times 50 \mathrm{~mL})$. The combined organic extracts were washed with $\mathrm{H}_{2} \mathrm{O}(1 \times 50 \mathrm{~mL})$, and brine $(1 \times 50 \mathrm{~mL})$, dried over $\mathrm{MgSO}_{4}$, filtered and concentrated under reduced pressure. Gradient flash chromatography (Petroleum ether/diethyl ether, 100:0 $\rightarrow$ 95:5 $\rightarrow$ 90:10) afforded 3d $(0.94 \mathrm{~g}, 92 \%)$ as a clear yellow oil: TLC $\mathrm{R}_{\mathrm{f}}=0.42\left(5 \% \mathrm{Et}_{2} \mathrm{O} / \mathrm{P} . \mathrm{E}\right.$.); IR (neat) $2955(\mathrm{~m})$, 2929 (m), 2884 (m), 2857 (m), 2227 (m), 2097 (m), 1634 (s), 1472 (m), 1386 (w), 1362 (w), 1329 (w), 1254 (s), 1207 (s), 1106 (s), 1056 (m), 1006 (s), 984 (m), 939 (w), 909 (m), 835 (s), 810 (s), 777 (s), 725 (s), 683 (s), 661 (s) cm ${ }^{-1} ;{ }^{1} \mathrm{H}$ NMR (400 MHz, CDCl N $_{3}$ ) $\delta 3.81\left(\mathrm{t}, J=6.6 \mathrm{~Hz}, 2 \mathrm{H}, \mathrm{C}_{1}-H_{2}\right), 3.26\left(\mathrm{~s}, 1 \mathrm{H}, \mathrm{C}_{7}-H\right), 2.63\left(\mathrm{t}, J=6.6 \mathrm{~Hz}, 2 \mathrm{H}, \mathrm{C}_{2}-H_{2}\right)$, $0.90\left(\mathrm{~s}, 9 \mathrm{H}, \mathrm{OSi}\left(\mathrm{CH}_{3}\right)_{2} \mathrm{C}\left(\mathrm{CH}_{3}\right)_{3}\right), 0.09\left(\mathrm{~s}, 6 \mathrm{H}, \mathrm{OSi}\left(\mathrm{CH}_{3}\right)_{2} \mathrm{C}\left(\mathrm{CH}_{3}\right)_{3}\right) ;{ }^{13} \mathrm{C} \mathrm{NMR}(100 \mathrm{MHz}$, $\left.\mathrm{CDCl}_{3}\right) \delta 160.2\left(\mathrm{C}_{5}\right), 93.8\left(\mathrm{C}_{3}\right), 82.5\left(\mathrm{C}_{4}\right), 82.2\left(\mathrm{C}_{6}\right), 78.2\left(\mathrm{C}_{7}\right), 60.5\left(\mathrm{C}_{1}\right), 25.8$ $\left(\mathrm{OSi}\left(\mathrm{CH}_{3}\right)_{2} \mathrm{C}\left(\mathrm{CH}_{3}\right)_{3}\right), 23.6\left(\mathrm{C}_{2}\right), 18.3\left(\mathrm{OSi}\left(\mathrm{CH}_{3}\right)_{2} \mathrm{C}\left(\mathrm{CH}_{3}\right)_{3}\right),-5.4\left(\mathrm{OSi}\left(\mathrm{CH}_{3}\right)_{2} \mathrm{C}\left(\mathrm{CH}_{3}\right)_{3}\right)$; high resolution mass spectrum $(+\mathrm{ESI}) \mathrm{m} / \mathrm{z} 259.1130\left[(\mathrm{M}+\mathrm{Na})^{+}\right.$; calcd for $\mathrm{C}_{13} \mathrm{H}_{20} \mathrm{O}_{2} \mathrm{SiNa}$ : 259.1130].

1-([1,3]Dithian-2-yl)-5-([1,3]dithian-2-yl)-7-(tert-butyl-dimethyl-silanyloxy)-heptan3-one (4d).

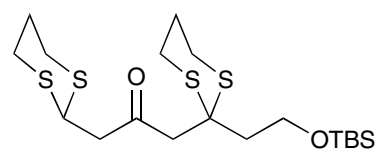

To a solution of 1-(tert-butyl-dimethyl-silanyloxy)-hepta-3,6-diyn-5-one (3d) (100 mg, $0.42 \mathrm{mmol})$ and propane-1,3-dithiol $(101 \mathrm{mg}, 0.93 \mathrm{mmol})$ in dichloromethane $(2 \mathrm{~mL})$ and methanol $(2 \mathrm{~mL})$ stirred at $-10{ }^{\circ} \mathrm{C}$ for $30 \mathrm{~min}$. was added sodium hydroxide $(37 \mathrm{mg}, 0.93$ $\mathrm{mmol}$ ). The reaction mixture was stirred for $30 \mathrm{~min}$. The reaction mixture was quenched with saturated aqueous $\mathrm{NH}_{4} \mathrm{Cl}$ solution $(5 \mathrm{~mL})$. The layers were separated, and the aqueous phase was extracted with diethyl ether $(3 \times 5 \mathrm{~mL})$. The combined organic extracts were washed with $\mathrm{H}_{2} \mathrm{O}(1 \times 5 \mathrm{~mL})$, and brine $(1 \times 5 \mathrm{~mL})$, dried over $\mathrm{MgSO}_{4}$, filtered and concentrated under reduced pressure. Gradient flash chromatography (Petroleum ether/diethyl ether, 100:0 $\rightarrow$ 90:10 $\rightarrow$ 80:20) afforded 4d (181 mg, 94\%) as a clear pale yellow oil: TLC $\mathrm{R}_{\mathrm{f}}=0.70$ (50\% $\mathrm{Et}_{2} \mathrm{O} / \mathrm{P} . \mathrm{E}$.); IR (neat) $2950(\mathrm{~m}), 2927(\mathrm{~m})$, 2897 (m), 2855 (m), 1717 (m), 1471 (m), 1422 (m), 1346 (m), 1276 (m), 1251 (s), 1173 (m), 1086 (s), 1005 (m), 968 (m), 939 (m), 908 (s), 834 (s), 776 (s), 731 (s), 663 (s) cm cm $^{-1}$ ${ }^{1} \mathrm{H}$ NMR $\left(400 \mathrm{MHz}, \mathrm{CDCl}_{3}\right) \delta 4.50\left(\mathrm{t}, J=6.9 \mathrm{~Hz}, 1 \mathrm{H}, \mathrm{C}_{1}-H\right), 3.86(\mathrm{t}, J=6.8 \mathrm{~Hz}, 2 \mathrm{H}$, $\left.\mathrm{C}_{7}-\mathrm{H}_{2}\right), 3.12\left(\mathrm{~s}, 2 \mathrm{H}, \mathrm{C}_{4}-\mathrm{H}_{2}\right), 2.93\left(\mathrm{~m}, 2 \mathrm{H}\right.$, two of $\left.\mathrm{SCH}_{2} \mathrm{CH}_{2} \mathrm{CH}_{2} \mathrm{~S}\right), 2.92(\mathrm{~d}, J=6.9 \mathrm{~Hz}, 2$ $\left.\mathrm{H}, \mathrm{C}_{2}-\mathrm{H}_{2}\right), 2.84\left(\mathrm{~m}, 6 \mathrm{H}\right.$, six of $\left.\mathrm{SCH}_{2} \mathrm{CH}_{2} \mathrm{CH}_{2} \mathrm{~S}\right), 2.36\left(\mathrm{t}, \mathrm{J}=6.8 \mathrm{~Hz}, 2 \mathrm{H}, \mathrm{C}_{6}-\mathrm{H}_{2}\right), 2.08(\mathrm{~m}$, $1 \mathrm{H}$, one of $\left.\mathrm{SCH}_{2} \mathrm{CH}_{2} \mathrm{CH}_{2} \mathrm{~S}\right), 1.88\left(\mathrm{~m}, 2 \mathrm{H}\right.$, two of $\left.\mathrm{SCH}_{2} \mathrm{CH}_{2} \mathrm{CH}_{2} \mathrm{~S}\right), 1.85(\mathrm{~m}, 1 \mathrm{H}$, one of $\left.\left.\mathrm{SCH}_{2} \mathrm{CH}_{2} \mathrm{CH}_{2} \mathrm{~S}\right), 0.89\left(\mathrm{~s}, 9 \mathrm{H}, \mathrm{OSi}\left(\mathrm{CH}_{3}\right)_{2} \mathrm{C}\left(\mathrm{CH}_{3}\right)_{3}\right), 0.06\left(\mathrm{~s}, 6 \mathrm{H}, \mathrm{OSi}\left(\mathrm{CH}_{3}\right)_{2} \mathrm{C}_{\left(\mathrm{CH}_{3}\right.}\right)_{3}\right) ;{ }^{13} \mathrm{C}$ NMR (100 MHz, CDCl $\left.{ }_{3}\right) \delta 201.7(\mathrm{C}), 59.7\left(\mathrm{C}_{7}\right), 59.7\left(\mathrm{C}_{5}\right) 51.2\left(\mathrm{C}_{4}\right), 49.7$ (one of $\mathrm{SCH}_{2} \mathrm{CH}_{2} \mathrm{CH}_{2} \mathrm{~S}$ ), $48.7\left(\mathrm{C}_{2}\right), 41.2\left(\mathrm{C}_{1}\right), 40.8\left(\mathrm{C}_{6}\right), 30.2$ (one of $\mathrm{SCH}_{2} \mathrm{CH}_{2} \mathrm{CH}_{2} \mathrm{~S}$ ), 26.5 (one

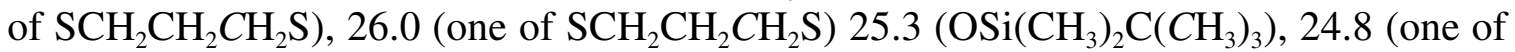
$\mathrm{SCH}_{2} \mathrm{CH}_{2} \mathrm{CH}_{2} \mathrm{~S}$ ), 24.8 (one of $\mathrm{SCH}_{2} \mathrm{CH}_{2} \mathrm{CH}_{2} \mathrm{~S}$ ), $18.3\left(\mathrm{OSi}\left(\mathrm{CH}_{3}\right)_{2} C\left(\mathrm{CH}_{3}\right)_{3}\right),-5.3$ 
$\left(\mathrm{OSi}\left(\mathrm{CH}_{3}\right)_{2} \mathrm{C}\left(\mathrm{CH}_{3}\right)_{3}\right)$; high resolution mass spectrum $(+\mathrm{ESI}) \mathrm{m} / \mathrm{z} 475.1265\left[(\mathrm{M}+\mathrm{Na})^{+}\right.$; calcd for $\mathrm{C}_{19} \mathrm{H}_{36} \mathrm{O}_{2} \mathrm{~S}_{4} \mathrm{SiNa}$ : 475.1265].

\section{2 (R) 3-Benzyloxy-2-methyl-propionaldehyde}

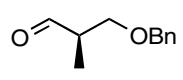

Under argon, a solution of oxalyl chloride $(7.04 \mathrm{~g}, 55.48 \mathrm{mmol})$ was stirred in dichloromethane $(100 \mathrm{~mL})$ at $-78{ }^{\circ} \mathrm{C}$ for $30 \mathrm{~min}$. To this solution was added dropwise dimethyl sulfoxide (DMSO) $(10.84 \mathrm{~g}, 138.70 \mathrm{mmol})$. The reaction mixture was stirred at $-78{ }^{\circ} \mathrm{C}$ for $10 \mathrm{~min}$., and a solution of $2(\mathrm{~S})$, 3-benzyloxy-2-methyl-propan-1-ol (5.00 g, $27.74 \mathrm{mmol})$ in dichloromethane $(20 \mathrm{~mL})$ was added dropwise. The reaction mixture was stirred at $-78{ }^{\circ} \mathrm{C}$ for $25 \mathrm{~min}$., then $N, N$-diisopropylthylamine (DIPEA) $(14.34 \mathrm{~g}$, $110.96 \mathrm{mmol}$ ) was added slowly and the reaction mixture was stirred at $-78{ }^{\circ} \mathrm{C}$ for 25 min., then allowed to warm to $-10{ }^{\circ} \mathrm{C}$ and stirred for $25 \mathrm{~min}$. The reaction mixture was quenched with a solution of $\mathrm{pH} 7$ phosphate buffer $(100 \mathrm{~mL})$. The reaction mixture was poured into diethyl ether $(400 \mathrm{~mL})$, washed with a saturated solution of $\mathrm{NH}_{4} \mathrm{Cl}(100 \mathrm{~mL})$, water $(100 \mathrm{~mL})$ and brine $(100 \mathrm{~mL})$, dried over $\mathrm{MgSO}_{4}$, filtered and concentrated under reduced pressure. Gradient flash chromatography (Petroleum ether/diethyl ether, 100:0 $\rightarrow$ 95:5 $\rightarrow$ 90:10 $\rightarrow$ 85:15 $\rightarrow$ 80:20) afforded the aldehyde (4.22 g, 77\%) as a clear pale yellow oil: $\mathrm{TLC} \mathrm{R}_{\mathrm{f}}=0.26\left(10 \% \mathrm{Et}_{2} \mathrm{O} / \mathrm{P} . \mathrm{E}\right.$.); $[\alpha]_{D}^{25}-0.8\left(c 1.055, \mathrm{CHCl}_{3}\right)$; IR (neat) 2860 (m), 2719 (w), 1721 (s), 1496 (m), 1454 (s), 1361 (m), 1247 (w), 1204 (m), 1094 (s), 1028 (s), 915 (m), 806 (m), 736 (s), 697 (s) cm ${ }^{-1} ;{ }^{1} \mathrm{H}$ NMR $\left(600 \mathrm{MHz}, \mathrm{CDCl}_{3}\right) \delta 9.74$ (d, J $\left.=1.1 \mathrm{~Hz}, 1 \mathrm{H}, \mathrm{C}_{1}-H\right), 7.32\left(\mathrm{~m}, 5 \mathrm{H}, \mathrm{Ph}-H_{5}\right), 4.53\left(\mathrm{~s}, 2 \mathrm{H}, \mathrm{CH}_{2} \mathrm{Ph}\right), 3.69(\mathrm{dd}, J=9.4,6.8$ $\mathrm{Hz}, 1 \mathrm{H}$, one of $\left.\mathrm{C}_{3}-H_{2}\right), 3.65\left(\mathrm{dd}, J=9.4,5.2 \mathrm{~Hz}, 1 \mathrm{H}\right.$, one of $\left.\mathrm{C}_{3}-H_{2}\right), 2.67\left(\mathrm{~m}, 1 \mathrm{H}, \mathrm{C}_{2}-H\right)$, $1.14\left(\mathrm{~d}, J=7.1 \mathrm{~Hz}, 3 \mathrm{H}, \mathrm{C}_{2} \mathrm{CH}_{3}\right) ;{ }^{13} \mathrm{C} \mathrm{NMR}\left(150 \mathrm{MHz}, \mathrm{CDCl}_{3}\right) 203.8\left(\mathrm{C}_{1}\right), 137.9(\mathrm{Ph})$, $128.4(\mathrm{Ph}), 127.7(\mathrm{Ph}), 127.6(\mathrm{Ph}), 73.3\left(\mathrm{CH}_{2} \mathrm{Ph}\right), 70.1\left(\mathrm{C}_{3}\right), 46.8\left(\mathrm{C}_{2}\right), 10.7\left(\mathrm{C}_{2} \mathrm{CH}_{3}\right)$; high resolution mass spectrum (+ESI) $m / z \quad 178.0985\left[\mathrm{M}^{+}\right]$; calcd for $\mathrm{C}_{11} \mathrm{H}_{14} \mathrm{O}_{2}$ : 178.2277]; spectra consistent with known data. ${ }^{3}$

\section{3 (S) 1,1-Dibromo-3-methyl-5-benzyloxybut-1-ene}

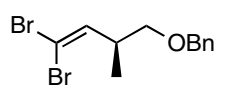

To a solution of triphenylphosphine $(10.61 \mathrm{~g}, 40.40 \mathrm{mmol})$ and carbon tetrabromide $(6.70$ $\mathrm{g}, 20.20 \mathrm{mmol})$ in dichloromethane $(100 \mathrm{~mL})$ stirred at $0{ }^{\circ} \mathrm{C}$ for $5 \mathrm{~min}$. was added a solution of $2(R)$, 3-benzyloxy-2-methyl-propionaldehyde $(2.00 \mathrm{~g}, 10.10 \mathrm{mmol})$ in dichloromethane $(10 \mathrm{~mL})$. The reaction mixture was stirred at $0{ }^{\circ} \mathrm{C}$ for $10 \mathrm{~min}$. The reaction mixture was quenched with saturated solution of aqueous $\mathrm{K}_{2} \mathrm{CO}_{3}$. The layers were separated, and the aqueous phase was extracted with dichloromethane $(3 \times 50 \mathrm{~mL})$. The combined organic extracts were washed with $\mathrm{H}_{2} \mathrm{O}(1 \times 50 \mathrm{~mL})$, and brine $(1 \times 50$

\footnotetext{
${ }^{3}$ Meyers, A. I.; Babiak, K. A.; Campbell, A. L.; Comins, D. L.; Fleming, M. P.; Henning, R.; Heuschmann, M; Hudspeth, J. P.; Kane, J. M.; Reider, P. J.; Roland, D. M.; Shimizu, K.; Tomioka, K; Walkup R. D. J. Am. Chem. Soc. 1983, 105, 5015
} 
$\mathrm{mL}$ ), dried over $\mathrm{MgSO}_{4}$, filtered and concentrated under reduced pressure. Gradient flash chromatography (Petroleum ether/diethyl ether, 100:0 $\rightarrow$ 95:5) afforded the dibromoolefin $(3.00 \mathrm{~g}, 89 \%)$ as a colorless oil: $\mathrm{TLC}_{\mathrm{f}}=0.74\left(10 \% \mathrm{Et}_{2} \mathrm{O} / \mathrm{P} . \mathrm{E}\right.$.); $[\alpha]_{D}^{25}+21.6(c$ 1.04, $\mathrm{CHCl}_{3}$ ); IR (neat) 3030 (w), $2964(\mathrm{~m}), 2931$ (m), 2854 (s), 2066 (w), 1616 (m), 1496 (s), 1453 (s), 1358 (s), 1233 (s), 1204 (s), 1096 (s), 1028 (s), 973 (m), 923(m), 906 (m), $843(\mathrm{~m}), 783$ (s), $734(\mathrm{~s}), 697$ (s) cm ${ }^{-1} ;{ }^{1} \mathrm{H} \mathrm{NMR}\left(400 \mathrm{MHz}, \mathrm{CDCl}_{3}\right) \delta 7.34(\mathrm{~m}, 5 \mathrm{H}$, $\left.\mathrm{Ph}_{5}\right), 6.33\left(\mathrm{~d}, J=9.1 \mathrm{~Hz}, 1 \mathrm{H}, \mathrm{C}_{3}-H\right), 4.53\left(\mathrm{~d}, J=2.0 \mathrm{~Hz}, 2 \mathrm{H}, \mathrm{CH}_{2} \mathrm{Ph}\right), 3.39(\mathrm{~m}, 2 \mathrm{H}$, $\left.\mathrm{C}_{1}-\mathrm{H}_{2}\right), 2.80\left(\mathrm{~m}, 1 \mathrm{H}, \mathrm{C}_{2}-H\right), 1.08\left(\mathrm{~d}, J=6.8 \mathrm{~Hz}, 3 \mathrm{H}, \mathrm{C}_{2} \mathrm{CH}_{3}\right) ;{ }^{13} \mathrm{C}$ NMR $(100 \mathrm{MHz}$, $\left.\mathrm{CDCl}_{3}\right) 141.2\left(\mathrm{C}_{3}\right), 138.3\left(\mathrm{C}_{2}\right), 128.4(\mathrm{Ph}), 127.7(\mathrm{Ph}), 127.5(\mathrm{Ph}), 88.9\left(\mathrm{C}_{4}\right), 73.1$ $\left(\mathrm{CH}_{2} \mathrm{Ph}\right), 73.0\left(\mathrm{C}_{1}\right), 38.8\left(\mathrm{C}_{2}\right), 15.9\left(\mathrm{C}_{2} \mathrm{CH}_{3}\right)$; high resolution mass spectrum $(+\mathrm{ESI}) \mathrm{m} / \mathrm{z}$ 333.9379 $\left[\mathrm{M}^{+}\right.$; calcd for $\mathrm{C}_{12} \mathrm{H}_{14} \mathrm{OBr}_{2}$ : 333.9370]; elemental analysis $\mathrm{C}, 43.43 \%$; $\mathrm{H}$, $4.30 \%$.

\section{4 (S) 5-Benzyloxy-4-methyl-pent-2-ynal}

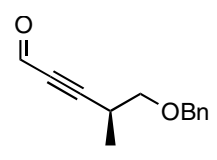

To a solution of $3(S)$ 1,1-dibromo-3-methyl-5-benzyloxybut-1-ene (2.00 g, $5.99 \mathrm{mmol})$ in tetrahydrofuran $(100 \mathrm{~mL})$ stirred at $-78{ }^{\circ} \mathrm{C}$ for $30 \mathrm{~min}$., was added a solution of $n \mathrm{BuLi}$ (1.6 $\mathrm{M}$ in hexane) $(9.36 \mathrm{~mL}, 14.97 \mathrm{mmol})$. The reaction mixture was stirred at $-78{ }^{\circ} \mathrm{C}$ for $1 \mathrm{~h}$. To the reaction mixture was added a solution of $N$-formylmorpholine $(0.97 \mathrm{~g}, 8.38$ $\mathrm{mmol})$ in tetrahydrofuran $(5 \mathrm{~mL})$. The reaction mixture was allowed to warm to ambient temperature over $2 \mathrm{~h}$. The reaction mixture was quenched with saturated solution of aqueous $\mathrm{NH}_{4} \mathrm{Cl}(100 \mathrm{~mL})$. The layers were separated, and the aqueous phase was extracted with diethyl ether $(3 \times 50 \mathrm{~mL})$. The combined organic extracts were washed with $\mathrm{H}_{2} \mathrm{O}(1 \times 50 \mathrm{~mL})$, and brine $(1 \times 50 \mathrm{~mL})$, dried over $\mathrm{MgSO}_{4}$, filtered and concentrated under reduced pressure. Gradient flash chromatography (Petroleum ether/diethyl ether, 100:0 $\rightarrow$ 95:5 $\rightarrow$ 90:10) afforded the ynal $(0.51 \mathrm{~g}, 42 \%)$ as a colorless oil: TLC $\mathrm{R}_{\mathrm{f}}=0.33\left(10 \% \mathrm{Et}_{2} \mathrm{O} / \mathrm{P} . \mathrm{E}\right.$.); $[\alpha]_{D}^{25}-24.2$ (c 1.07, $\mathrm{CHCl}_{3}$ ); IR (neat) $3032(\mathrm{w})$, 2860 (s), 2211 (s), 1663 (s), 1496 (m), 1454 (s), 1388 (m), 1360 (m), 1334 (m), 1260 (m), 1203 (m), 1164 (s), 1119 (s), 1096 (s), 1028 (s), 957 (m), 887 (s), 793 (s), 739 (s), 699 (s) $\mathrm{cm}^{-1} ;{ }^{1} \mathrm{H}$ NMR $\left(400 \mathrm{MHz}, \mathrm{CDCl}_{3}\right) \delta 9.20\left(\mathrm{~d}, J=0.7 \mathrm{~Hz}, 1 \mathrm{H}, \mathrm{C}_{1}-H\right), 7.35\left(\mathrm{~m}, 5 \mathrm{H}, \mathrm{PhH}_{5}\right)$, $4.57\left(\mathrm{~s}, 2 \mathrm{H}, \mathrm{CH}_{2} \mathrm{Ph}\right), 3.56\left(\mathrm{dd}, J=9.1,6.6 \mathrm{~Hz}, 1 \mathrm{H}\right.$, one of $\left.\mathrm{C}_{5}-\mathrm{H}_{2}\right), 3.46(\mathrm{dd}, J=9.1,6.6$ $\mathrm{Hz}, 1 \mathrm{H}$, one of $\left.\mathrm{C}_{5}-\mathrm{H}_{2}\right), 2.96\left(\mathrm{q}, J=6.8 \mathrm{~Hz}, 1 \mathrm{H}, \mathrm{C}_{4}-H\right), 1.28\left(\mathrm{~d}, J=7.0 \mathrm{~Hz}, 3 \mathrm{H}, \mathrm{C}_{4} \mathrm{CH}_{3}\right)$; ${ }^{13} \mathrm{C}$ NMR $\left(100 \mathrm{MHz}, \mathrm{CDCl}_{3}\right) \delta 177.0\left(\mathrm{C}_{1}\right), 137.8(\mathrm{Ph}), 128.5(\mathrm{Ph}), 127.8(\mathrm{Ph}), 127.7$ $(\mathrm{Ph}), 100.0\left(\mathrm{C}_{3}\right), 81.9\left(\mathrm{C}_{2}\right), 73.2\left(\mathrm{CH}_{2} \mathrm{Ph}\right), 72.7\left(\mathrm{C}_{5}\right), 27.3\left(\mathrm{C}_{4}\right), 16.6\left(\mathrm{C}_{4} C_{3}\right)$; high resolution mass spectrum (+ESI) $\mathrm{m} / z 202.0999$ [ $\mathrm{M}^{+}$; calcd for $\mathrm{C}_{13} \mathrm{H}_{14} \mathrm{O}_{2}$ : 202.0994]; elemental analysis C, 77.14\%; $\mathrm{H}, 7.02 \%$.

\section{2 (S) 1-Benzyloxy-2-methyl-hepta-3,6-diyn-5-ol}

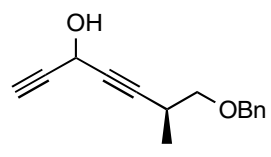


To a solution of $4(S)$ 5-benzyloxy-4-methyl-pent-2-ynal $(0.51 \mathrm{~g}, 2.53 \mathrm{mmol})$ in tetrahydrofuran $(20 \mathrm{~mL})$ stirred at $-78{ }^{\circ} \mathrm{C}$ for $30 \mathrm{~min}$. was added a solution of ethynylmagnesium bromide $(0.5 \mathrm{M}$ in tetrahydrofuran) $(20.26 \mathrm{~mL}, 10.13 \mathrm{mmol})$. The reaction mixture was allowed to warm to $-30{ }^{\circ} \mathrm{C}$ and stirred for $30 \mathrm{~min}$. The reaction mixture was quenched with saturated solution of aqueous $\mathrm{NH}_{4} \mathrm{Cl}(10 \mathrm{~mL})$. The layers were separated, and the aqueous phase was extracted with diethyl ether $(3 \times 10 \mathrm{~mL})$. The combined organic extracts were washed with $\mathrm{H}_{2} \mathrm{O}(1 \times 10 \mathrm{~mL})$, and brine $(1 \times 10 \mathrm{~mL})$, dried over $\mathrm{MgSO}_{4}$, filtered and concentrated under reduced pressure. Gradient flash chromatography (Petroleum ether/diethyl ether, 100:0 $\rightarrow$ 90:10 $\rightarrow$ 70:30) afforded the bis-ynol $(0.41 \mathrm{~g}, 72 \%)$ as a clear yellow oil: TLC $\mathrm{R}_{\mathrm{f}}=0.05\left(10 \% \mathrm{Et}_{2} \mathrm{O} / \mathrm{P} . \mathrm{E}\right.$.); IR (neat) 3285 (br, m), 3032 (m), 2974 (m), 2864 (m), 2243 (w), 2122 (w), 1496 (m), $1454(\mathrm{~s})$, 1362 (s), 1298 (s), 1205 (m), 1166 (m), 1123 (s), 1092 (s), 1074 (s), 1017 (s), 919 (s), $781(\mathrm{~m}), 736(\mathrm{~s}), 697(\mathrm{~s}) \mathrm{cm}^{-1} ;{ }^{1} \mathrm{H} \mathrm{NMR}\left(600 \mathrm{MHz}, \mathrm{CDCl}_{3}\right) \delta 7.35\left(\mathrm{~m}, 5 \mathrm{H}, \mathrm{Ph} H_{5}\right), 5.10$ $\left(\mathrm{d}, J=6.5 \mathrm{~Hz}, 1 \mathrm{H}, \mathrm{C}_{5}-\mathrm{H}\right), 4.57\left(\mathrm{~s}, 2 \mathrm{H}, \mathrm{CH}_{2} \mathrm{Ph}\right), 3.53\left(\mathrm{dd}, J=9.1,6.3 \mathrm{~Hz}, 1 \mathrm{H}\right.$, one of $\mathrm{C}_{1^{-}}$ $\left.H_{2}\right), 3.39\left(\mathrm{dd}, J=8.9,8.9 \mathrm{~Hz}, 1 \mathrm{H}\right.$, one of $\left.\mathrm{C}_{1}-H_{2}\right), 2.80\left(\mathrm{~m}, 1 \mathrm{H}, \mathrm{C}_{2}-H\right), 2.54\left(\mathrm{~m}, 1 \mathrm{H}, \mathrm{C}_{7^{-}}\right.$ $H), 1.22\left(\mathrm{~d}, J=6.9 \mathrm{~Hz}, 3 \mathrm{H}, \mathrm{C}_{2} \mathrm{CH}_{3}\right) ;{ }^{13} \mathrm{C}$ NMR $\left(150 \mathrm{MHz}, \mathrm{CDCl}_{3}\right) \delta 138.1(\mathrm{Ph}), 138.1$ (Ph'), $128.4(\mathrm{Ph}), 127.7(\mathrm{Ph}), 127.6(\mathrm{Ph}), 87.3\left(\mathrm{C}_{3}\right), 81.4\left(\mathrm{C}_{7}\right), 78.0\left(\mathrm{C}_{4}\right), 73.5\left(\mathrm{C}_{1}\right), 73.0$ $\left(\mathrm{CH}_{2} \mathrm{Ph}\right), 72.2\left(\mathrm{C}_{6}\right), 72.2\left(\mathrm{C}_{6}{ }_{6}\right), 52.1\left(\mathrm{C}_{5}\right), 26.7\left(\mathrm{C}_{2}\right), 17.3\left(\mathrm{C}_{2} \mathrm{CH}_{3}\right)$; high resolution mass spectrum (+ESI) $m / z 251.1048\left[(\mathrm{M}+\mathrm{Na})^{+}\right.$; calcd for $\mathrm{C}_{15} \mathrm{H}_{16} \mathrm{O}_{2} \mathrm{Na}$ : 251.1048].

\section{2 (S) 1-Benzyloxy-2-methyl-hepta-3,6-diyn-5-one (3e).}

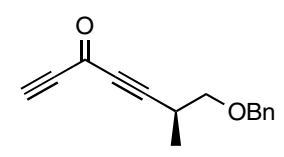

To a solution of $2(S)$ 1-benzyloxy-2-methyl-hepta-3,6-diyn-5-ol (200 mg, $0.88 \mathrm{mmol})$ in dichloromethane $(5 \mathrm{~mL})$ stirred at $0{ }^{\circ} \mathrm{C}$ for $30 \mathrm{~min}$. was added Dess-Martin periodinane $(0.56 \mathrm{~g}, 1.31 \mathrm{mmol})$. The reaction mixture was allowed to warm to ambient temperature and stirred for $1 \mathrm{~h}$. The reaction mixture was quenched with a 1:1 solution of saturated aqueous $\mathrm{Na}_{2} \mathrm{~S}_{2} \mathrm{O}_{3}$ and saturated aqueous $\mathrm{NaHCO}_{3}(10 \mathrm{~mL})$. The layers were separated, and the aqueous phase was extracted with diethyl ether $(3 \times 10 \mathrm{~mL})$. The combined organic extracts were washed with $\mathrm{H}_{2} \mathrm{O}(1 \times 10 \mathrm{~mL})$, and brine $(1 \times 10 \mathrm{~mL})$, dried over $\mathrm{MgSO}_{4}$, filtered and concentrated under reduced pressure. Gradient flash chromatography (Petroleum ether/diethyl ether, 100:0 $\rightarrow$ 90:10 $\rightarrow$ 80:20) afforded 3e (123 mg, 62\%) as a clear yellow oil: TLC $\mathrm{R}_{\mathrm{f}}=0.42$ (20\% $\left.\mathrm{Et}_{2} \mathrm{O} / \mathrm{P} . \mathrm{E}.\right) ;{ }^{1} \mathrm{H}$ NMR (400 $\left.\mathrm{MHz}, \mathrm{CDCl}_{3}\right) \delta 7.35\left(\mathrm{~m}, 5 \mathrm{H}, \mathrm{Ph}-H_{5}\right), 4.58\left(\mathrm{~s}, 2 \mathrm{H}, \mathrm{CH}_{2} \mathrm{Ph}\right), 3.57(\mathrm{dd}, J=9.1,6.5 \mathrm{~Hz}, 1$ $\mathrm{H}$, one of $\left.\mathrm{C}_{1}-H_{2}\right), 3.47\left(\mathrm{dd}, J=9.1,6.8 \mathrm{~Hz}, 1 \mathrm{H}\right.$, one of $\left.\mathrm{C}_{1}-H_{2}\right), 3.27\left(\mathrm{~s}, 1 \mathrm{H}, \mathrm{C}_{7}-H\right), 2.96$ $\left(\mathrm{m}, 1 \mathrm{H}, \mathrm{C}_{2}-H\right), 1.29\left(\mathrm{~d}, J=7.0 \mathrm{~Hz}, 3 \mathrm{H}, \mathrm{C}_{2} \mathrm{CH}_{3}\right)$.

6 (R) 1-([1,3]Dithian-2-yl)-5-([1,3]dithian-2-yl)-6-methyl-7-benzyloxy-heptan-3-one (4e). 


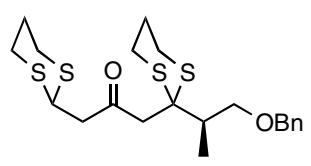

To a solution of $2(S)$ 1-benzyloxy-2-methyl-hepta-3,6-diyn-5-one (3e). (190 mg, 0.84 $\mathrm{mmol})$ and propane-1,3-dithiol $(200 \mathrm{mg}, 1.85 \mathrm{mmol})$ in dichloromethane $(8 \mathrm{~mL})$ and methanol $(8 \mathrm{~mL})$ stirred at $-10{ }^{\circ} \mathrm{C}$ for $30 \mathrm{~min}$. was added sodium methoxide $(100 \mathrm{mg}$, $1.85 \mathrm{mmol})$. The reaction mixture was allowed to warm to ambient temperature over 18 h. The reaction mixture was quenched with saturated aqueous $\mathrm{NH}_{4} \mathrm{Cl}$ solution $(10 \mathrm{~mL})$. The layers were separated, and the aqueous phase was extracted with diethyl ether $(3 \times$ $10 \mathrm{~mL})$. The combined organic extracts were washed with $\mathrm{H}_{2} \mathrm{O}(1 \times 10 \mathrm{~mL})$, and brine $(1$ $\times 10 \mathrm{~mL}$ ), dried over $\mathrm{MgSO}_{4}$, filtered and concentrated under reduced pressure. Gradient flash chromatography (Petroleum ether/diethyl ether, 100:0 $\rightarrow$ 90:10 $\rightarrow$ 80:20 $\rightarrow$ 70:30 $\rightarrow$ 60:40) afforded $4 \mathrm{e}(314 \mathrm{mg}, 84 \%)$ as a yellow oil: $\mathrm{TLC} \mathrm{R}_{\mathrm{f}}=0.47\left(50 \% \mathrm{Et}_{2} \mathrm{O} / \mathrm{P} . \mathrm{E}.\right) ;[\alpha]_{D}^{25}$ +30.6 (c 1.10, $\mathrm{CHCl}_{3}$ ); IR (neat) 2898 (s), $1717(\mathrm{~s}), 1496(\mathrm{~m}), 1452$ (s), 1421 (s), 1346 (s), 1276 (s), 1242 (s), 1173 (m), 1088 (s), 1027 (s), 984 (s), 907 (s), 870 (m), 815 (m), 733 (s), 698 (s), $676(\mathrm{~m}) \mathrm{cm}^{-1} ;{ }^{1} \mathrm{H}$ NMR (400 MHz, $\left.\mathrm{CDCl}_{3}\right) \delta 7.33\left(\mathrm{~m}, 4 \mathrm{H}, \mathrm{Ph} H_{4}\right), 7.27$ $(\mathrm{m}, 1 \mathrm{H}, \mathrm{Ph} H), 4.52\left(\mathrm{~d}, J=11.7 \mathrm{~Hz}, 1 \mathrm{H}\right.$, one of $\left.\mathrm{CH}_{2} \mathrm{Ph}\right), 4.48\left(\mathrm{t}, J=7.0 \mathrm{~Hz}, 1 \mathrm{H}, \mathrm{C}_{1}-H\right)$, $4.47\left(\mathrm{~d}, J=11.7 \mathrm{~Hz}, 1 \mathrm{H}\right.$, one of $\left.\mathrm{CH}_{2} \mathrm{Ph}\right), 3.96\left(\mathrm{dd}, J=9.4,4.3 \mathrm{~Hz}, 1 \mathrm{H}\right.$, one of $\left.\mathrm{C}_{7}-H_{2}\right)$, $3.48\left(\mathrm{dd}, J=9.4,7.3 \mathrm{~Hz}, 1 \mathrm{H}\right.$, one of $\left.\mathrm{C}_{7}-H_{2}\right), 3.29\left(\mathrm{~d}, J=15.6 \mathrm{~Hz}, 1 \mathrm{H}\right.$, one of $\left.\mathrm{C}_{4}-H_{2}\right)$, $3.15\left(\mathrm{~d}, J=15.6 \mathrm{~Hz}, 1 \mathrm{H}\right.$, one of $\left.\mathrm{C}_{4}-H_{2}\right), 2.89\left(\mathrm{~m}, 2 \mathrm{H}, \mathrm{C}_{2}-H_{2}\right), 2.79(\mathrm{~m}, 7 \mathrm{H}$, $\left.\mathrm{SCH}_{2} \mathrm{CH}_{2} \mathrm{CH}_{2} \mathrm{~S}\right), 2.68\left(\mathrm{~m}, 1 \mathrm{H}, \mathrm{C}_{6}-\mathrm{H}\right), 2.08\left(\mathrm{~m}, 1 \mathrm{H}\right.$, one of $\left.\mathrm{SCH}_{2} \mathrm{CH}_{2} \mathrm{CH}_{2} \mathrm{~S}\right), 2.00(\mathrm{~m}, 1$ $\mathrm{H}$, one of $\left.\mathrm{SCH}_{2} \mathrm{CH}_{2} \mathrm{CH}_{2} \mathrm{~S}\right), 1.89\left(\mathrm{~m}, 1 \mathrm{H}\right.$, one of $\left.\mathrm{SCH}_{2} \mathrm{CH}_{2} \mathrm{CH}_{2} \mathrm{~S}\right), 1.87(\mathrm{~m}, 1 \mathrm{H}$, one of $\left.\mathrm{SCH}_{2} \mathrm{CH}_{2} \mathrm{CH}_{2} \mathrm{~S}\right), 1.25\left(\mathrm{~d}, J=6.9 \mathrm{~Hz}, 3 \mathrm{H}, \mathrm{C}_{6} \mathrm{CH}_{3}\right) ;{ }^{13} \mathrm{C} \mathrm{NMR}\left(100 \mathrm{MHz}, \mathrm{CDCl}_{3}\right) \delta 202.2$ $\left(\mathrm{C}_{3}\right), 138.5(\mathrm{Ph}), 128.3(\mathrm{Ph}), 127.6(\mathrm{Ph}), 127.4(\mathrm{Ph}), 73.0\left(\mathrm{CH}_{2} \mathrm{Ph}\right), 72.2\left(\mathrm{C}_{7}\right), 53.8\left(\mathrm{C}_{5}\right)$, $49.6\left(\mathrm{C}_{2}\right), 49.2\left(\mathrm{C}_{4}\right), 41.3\left(\mathrm{C}_{1}\right), 40.1\left(\mathrm{C}_{6}\right), 30.2$ (one of $\mathrm{SCH}_{2} \mathrm{CH}_{2} \mathrm{CH}_{2} \mathrm{~S}$ ), 26.4 (one of $\mathrm{SCH}_{2} \mathrm{CH}_{2} \mathrm{CH}_{2} \mathrm{~S}$ ), 26.4 (one of $\mathrm{SCH}_{2} \mathrm{CH}_{2} \mathrm{CH}_{2} \mathrm{~S}$ ), 26.2 (one of $\mathrm{SCH}_{2} \mathrm{CH}_{2} \mathrm{CH}_{2} \mathrm{~S}$ ), 25.3 (one of $\mathrm{SCH}_{2} \mathrm{CH}_{2} \mathrm{CH}_{2} \mathrm{~S}$ ), 24.7 (one of $\mathrm{SCH}_{2} \mathrm{CH}_{2} \mathrm{CH}_{2} \mathrm{~S}$ ), $13.5\left(\mathrm{C}_{6} \mathrm{CH}_{3}\right)$; high resolution mass spectrum (+ESI) $m / z$ 465.1026 [(M+Na) ${ }^{+}$; calcd for $\mathrm{C}_{21} \mathrm{H}_{30} \mathrm{O}_{2} \mathrm{~S}_{4} \mathrm{Na}$ : 465.1026].

\section{4(R)-2-(2,2-Diethyl-[1,3]dioxolan-4-yl)-ethanol}

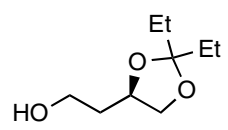

To a solution of $(R)$-1,2,4-butanetriol (5) (6.76 g, $63.68 \mathrm{mmol})$ in freshly distilled pentan3 -one $(320 \mathrm{~mL})$ was added $p$-toluenesulfonic acid ( $p$ TSA) $(6.06 \mathrm{~g}, 31.84 \mathrm{mmol})$. The reaction mixture was stirred at $53{ }^{\circ} \mathrm{C}$ for $16 \mathrm{~h}$, then triethylamine $(10 \mathrm{~mL})$ was added and the reaction mixture stirred at ambient temperature for $10 \mathrm{~min}$. The reaction mixture was concentrated under reduced pressure. Gradient flash chromatography (Dichloromethane/methanol, 100:0 $\rightarrow$ 97:3 $\rightarrow$ 95:5) afforded the protected alcohol $(9.84$ $\mathrm{g}, 89 \%)$ as a colorless oil: $\mathrm{TLC} \mathrm{R}_{\mathrm{f}}=0.50\left(5 \% \mathrm{MeOH} / \mathrm{CH}_{2} \mathrm{Cl}_{2}\right) ;[\alpha]_{D}^{25}-2.6(c 0.935$, $\mathrm{CHCl}_{3}$ ); IR (neat) 3416 (br, m), 2972 (s), 2939 (s), 2881 (s), 1464 (s), 1355 (s), 1294 (m), 1205 (s), 1172 (s), 1134 (s), 1078 (s), 1055 (s), 991 (s), 959 (s), 916 (s), 764 (s) cm ${ }^{-1} ;{ }^{1} \mathrm{H}$ NMR $\left(400 \mathrm{MHz}, \mathrm{CDCl}_{3}\right.$ ) $\delta 4.24\left(\operatorname{app} q n, J=7.2 \mathrm{~Hz}, 1 \mathrm{H}, \mathrm{C}_{4},-H\right), 4.09$ (dd, $J=8.0,6.3$ 
$\left.\mathrm{Hz}, 1 \mathrm{H}, \mathrm{C}_{5},-H\right), 3.80\left(\mathrm{~s}, 2 \mathrm{H}, \mathrm{C}_{1}-H_{2}\right), 3.54\left(\mathrm{t}, J=8.0 \mathrm{~Hz}, 1 \mathrm{H}, \mathrm{C}_{5},-H\right), 2.30\left(\mathrm{~s}, 1 \mathrm{H}, \mathrm{C}_{1} \mathrm{OH}\right)$, $1.81\left(\mathrm{~m}, 2 \mathrm{H}, \mathrm{C}_{2}-H_{2}\right), 1.65\left(\mathrm{q}, J=7.4 \mathrm{~Hz}, 2 \mathrm{H}, \mathrm{CH}_{2} \mathrm{CH}_{3}\right), 1.62(\mathrm{q}, J=7.4 \mathrm{~Hz}, 2 \mathrm{H}$, $\left.\mathrm{CH}_{2} \mathrm{CH}_{3}\right), 0.90\left(\mathrm{t}, J=7.4 \mathrm{~Hz}, 3 \mathrm{H}, \mathrm{CH}_{2} \mathrm{CH}_{3}\right), 0.89\left(\mathrm{t}, J=7.4 \mathrm{~Hz}, 3 \mathrm{H}, \mathrm{CH}_{2} \mathrm{CH}_{3}\right) ;{ }^{13} \mathrm{C} \mathrm{NMR}$ $\left(100 \mathrm{MHz}, \mathrm{CDCl}_{3}\right) \quad \delta 113.0\left(\mathrm{C}_{2}\right), 75.5\left(\mathrm{C}_{4}\right), 70.1\left(\mathrm{C}_{5}\right), 60.8\left(\mathrm{C}_{1}\right), 35.4\left(\mathrm{C}_{2}\right), 29.9$ $\left(\mathrm{CH}_{2} \mathrm{CH}_{3}\right), 29.60\left(\mathrm{CH}_{2} \mathrm{CH}_{3}\right), 8.2\left(\mathrm{CH}_{2} \mathrm{CH}_{3}\right), 7.9\left(\mathrm{CH}_{2} \mathrm{CH}_{3}\right)$; high resolution mass spectrum $(+\mathrm{ESI}) \mathrm{m} / z$ 197.1154 [(M+Na) ${ }^{+}$; calcd for $\mathrm{C}_{9} \mathrm{H}_{18} \mathrm{O}_{3} \mathrm{Na}$ : 197.1154].

\section{4(R) (2,2-Diethyl-[1,3]dioxolan-4-yl)-acetaldehyde}

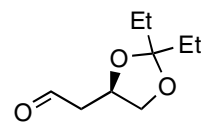

To a solution of 2(R)-2-(2,2-diethyl-[1,3]dioxolan-4-yl)-ethanol (4.00 g, $22.96 \mathrm{mmol})$ and $4 \AA$ A molecular sieves $(11.50 \mathrm{~g})$ in dichloromethane $(200 \mathrm{~mL})$ stirred at $0{ }^{\circ} \mathrm{C}$ for $5 \mathrm{~min}$. was added pyridinium chlorochromate (PCC) $(9.90 \mathrm{~g}, 45.92 \mathrm{mmol})$. The reaction mixture was allowed to warm to ambient temperature and stirred for $1 \mathrm{~h}$. The crude reaction mixture was filtered through a plug of silica, washed with diethyl ether $(50 \mathrm{~mL})$ and concentrated under reduced pressure to afford the aldehyde $(3.56 \mathrm{~g}, 90 \%)$ as a colorless oil: $\mathrm{TLC} \mathrm{R}_{\mathrm{f}}=0.60\left(5 \% \mathrm{MeOH} / \mathrm{CH}_{2} \mathrm{Cl}_{2}\right) ;{ }^{1} \mathrm{H} \mathrm{NMR}\left(400 \mathrm{MHz}, \mathrm{CDCl}_{3}\right) \delta 9.18(\mathrm{~s}$, $\left.1 \mathrm{H}, \mathrm{C}_{1}-H\right), 4.30\left(\mathrm{~m}, 1 \mathrm{H}, \mathrm{C}_{4}-\mathrm{H}\right), 4.15\left(\mathrm{dd}, J=8.1,6.1 \mathrm{~Hz}, 1 \mathrm{H}\right.$, one of $\left.\mathrm{C}_{5}-H_{2}\right), 3.72(\mathrm{dd}$, $J=8.1,6.8 \mathrm{~Hz}, 1 \mathrm{H}$, one of $\left.\mathrm{C}_{5},-H_{2}\right), 2.78\left(\mathrm{dd}, J=17.3,5.2 \mathrm{~Hz}, 1 \mathrm{H}\right.$, one of $\left.\mathrm{C}_{2}-H_{2}\right), 2.68$ $\left(\mathrm{dd}, J=17.3,6.7 \mathrm{~Hz}, 1 \mathrm{H}\right.$, one of $\left.\mathrm{C}_{2}-\mathrm{H}_{2}\right), 1.68\left(\mathrm{q}, J=7.5 \mathrm{~Hz}, 2 \mathrm{H}, \mathrm{CH}_{2} \mathrm{CH}_{3}\right), 1.63(\mathrm{q}, J=$ $\left.7.5 \mathrm{~Hz}, 2 \mathrm{H}, \mathrm{CH}_{2} \mathrm{CH}_{3}\right), 0.93\left(\mathrm{t}, J=7.5 \mathrm{~Hz}, 3 \mathrm{H}, \mathrm{CH}_{2} \mathrm{CH}_{3}\right), 0.90(\mathrm{t}, J=7.5 \mathrm{~Hz}, 3 \mathrm{H}$, $\left.\mathrm{CH}_{2} \mathrm{CH}_{3}\right)$; high resolution mass spectrum (+ESI) $\mathrm{m} / \mathrm{z} 195.0997\left[(\mathrm{M}+\mathrm{Na})^{+}\right.$; calcd for $\mathrm{C}_{9} \mathrm{H}_{16} \mathrm{O}_{3} \mathrm{Na}$ : 195.0997].

4(R) 4-(2,2-Diethyl-[1,3]dioxolan-4-yl)-1,1-dibromoprop-1-ene (6).

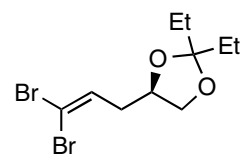

To a solution of triphenylphosphine $(2.47 \mathrm{~g}, 9.41 \mathrm{mmol})$ and carbon tetrabromide $(1.56 \mathrm{~g}$, $4.70 \mathrm{mmol})$ in dichloromethane $(20 \mathrm{~mL})$ stirred at $0{ }^{\circ} \mathrm{C}$ for $5 \mathrm{~min}$. was added a solution of $2(R)$ (2,2-diethyl-[1,3]dioxolan-4-yl)-acetaldehyde $(0.50 \mathrm{~g}, 2.35 \mathrm{mmol})$ in dichloromethane $(5 \mathrm{~mL})$. The reaction mixture was stirred at $0{ }^{\circ} \mathrm{C}$ for $5 \mathrm{~min}$. The reaction mixture was quenched with saturated solution of aqueous $\mathrm{K}_{2} \mathrm{CO}_{3}$. The layers were separated, and the aqueous phase was extracted with dichloromethane $(3 \times 50 \mathrm{~mL})$. The combined organic extracts were washed with $\mathrm{H}_{2} \mathrm{O}(1 \times 50 \mathrm{~mL})$, and brine $(1 \times 50$ $\mathrm{mL}$ ), dried over $\mathrm{MgSO}_{4}$, filtered and concentrated under reduced pressure. Gradient flash chromatography (Petroleum ether/diethyl ether, 100:0 $\rightarrow$ 98:2 $\rightarrow$ 96:4 $\rightarrow$ 94:6) afforded $6(0.64 \mathrm{~g}, 82 \%)$ as a colorless oil: TLC $\mathrm{R}_{\mathrm{f}}=0.56\left(10 \% \mathrm{Et}_{2} \mathrm{O} / \mathrm{P} . \mathrm{E}.\right) ;[\alpha]_{D}^{25}-1.9$ (c 0.365 , $\mathrm{CHCl}_{3}$ ); IR (neat) $2972(\mathrm{~m}), 2937$ (m), $2880(\mathrm{~m}), 1621$ (w), $1463(\mathrm{~m}), 1355$ (m), 1261 (m), 1228 (m), $1196(\mathrm{~m}), 1171$ (m), 1077 (s), 1058 (s), 965 (m), 923 (m), 881 (m), 782 (s) $\mathrm{cm}^{-1} ;{ }^{1} \mathrm{H} \mathrm{NMR}\left(400 \mathrm{MHz}, \mathrm{CDCl}_{3}\right) \delta 6.50\left(\mathrm{t}, J=7.1 \mathrm{~Hz}, 1 \mathrm{H}, \mathrm{C}_{2}-H\right), 4.19\left(\mathrm{~m}, 1 \mathrm{H}, \mathrm{C}_{4}\right.$,- 
$H$ ), $4.06\left(\mathrm{dd}, J=7.9,6.2 \mathrm{~Hz}, 1 \mathrm{H}\right.$, one of $\left.\mathrm{C}_{5},-H_{2}\right), 3.55\left(\mathrm{t}, J=7.7 \mathrm{~Hz}, 1 \mathrm{H}\right.$, one of $\left.\mathrm{C}_{5},-H_{2}\right)$, $2.39\left(\operatorname{app~t}, J=6.5 \mathrm{~Hz}, 2 \mathrm{H}, \mathrm{C}_{3}-H_{2}\right), 1.66\left(\mathrm{q}, J=7.5 \mathrm{~Hz}, 2 \mathrm{H}, \mathrm{CH}_{2} \mathrm{CH}_{3}\right), 1.62(\mathrm{q}, J=7.5$ $\left.\mathrm{Hz}, 2 \mathrm{H}, \mathrm{CH}_{2} \mathrm{CH}_{3}\right), 0.92\left(\mathrm{t}, J=7.5 \mathrm{~Hz}, 3 \mathrm{H}, \mathrm{CH}_{2} \mathrm{CH}_{3}\right), 0.90\left(\mathrm{t}, J=7.5 \mathrm{~Hz}, 3 \mathrm{H}, \mathrm{CH}_{2} \mathrm{CH}_{3}\right)$; ${ }^{13} \mathrm{C}$ NMR $\left(150 \mathrm{MHz}, \mathrm{CDCl}_{3}\right) \delta 134.0\left(\mathrm{C}_{2}\right), 113.3\left(\mathrm{C}_{2}\right), 90.9\left(\mathrm{C}_{1}\right), 74.0\left(\mathrm{C}_{4}\right), 69.2\left(\mathrm{C}_{5}\right)$, $37.0\left(\mathrm{C}_{3}\right), 29.8\left(\mathrm{CH}_{2} \mathrm{CH}_{3}\right), 29.4\left(\mathrm{CH}_{2} \mathrm{CH}_{3}\right), 8.2\left(\mathrm{CH}_{2} \mathrm{CH}_{3}\right), 7.9\left(\mathrm{CH}_{2} \mathrm{CH}_{3}\right)$; high resolution mass spectrum (+ESI) $\mathrm{m} / z$ [ $\left[\mathrm{M}^{+}\right.$; calcd for $\mathrm{C}_{10} \mathrm{H}_{16} \mathrm{O}_{2} \mathrm{Na}$ : 350.9394].

4'(R) 4-(2',2'-Diethyl-[1',3']dioxolan-4'-yl)-but-2-ynal (7).

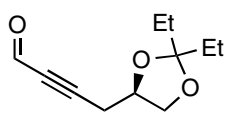

To a solution of 4(R) 4-(2,2-diethyl-[1,3]dioxolan-4-yl)-1,1-dibromoprop-1-ene (6) (1.50 $\mathrm{g}, 4.56 \mathrm{mmol})$ in tetrahydrofuran $(100 \mathrm{~mL})$ stirred at $-78{ }^{\circ} \mathrm{C}$ for $30 \mathrm{~min}$. was added a solution of $n \mathrm{BuLi}$ (1.6 $\mathrm{M}$ in hexane) $(7.13 \mathrm{~mL}, 11.40 \mathrm{mmol})$. The reaction mixture was stirred at $-78{ }^{\circ} \mathrm{C}$ for $1 \mathrm{~h}$. To the reaction mixture was added a solution of $\mathrm{N}$ formylmorpholine $(0.74 \mathrm{~g}, 6.39 \mathrm{mmol})$ in tetrahydrofuran $(5 \mathrm{~mL})$. The reaction mixture was allowed to warm to ambient temperature over $17 \mathrm{~h}$. The reaction mixture was quenched with saturated solution of aqueous $\mathrm{NH}_{4} \mathrm{Cl}(100 \mathrm{~mL})$. The layers were separated, and the aqueous phase was extracted with diethyl ether $(3 \times 100 \mathrm{~mL})$. The combined organic extracts were washed with $\mathrm{H}_{2} \mathrm{O}(1 \times 100 \mathrm{~mL})$, and brine $(1 \times 100 \mathrm{~mL})$, dried over $\mathrm{MgSO}_{4}$, filtered and concentrated under reduced pressure. Gradient flash chromatography (Petroleum ether/diethyl ether, 100:0 $\rightarrow$ 95:5 $\rightarrow$ 90:10 $\rightarrow$ 80:20) afforded $7(0.62 \mathrm{~g}, 70 \%)$ as a colorless oil: TLC $\mathrm{R}_{\mathrm{f}}=0.16\left(10 \% \mathrm{Et}_{2} \mathrm{O} /\right.$ P.E. $)$; $[\alpha]_{D}^{25}-37.7$ (c 0.345, $\mathrm{CHCl}_{3}$ ); IR (neat) $2973(\mathrm{~m}), 2939(\mathrm{~m}), 2883(\mathrm{~m}), 2280(\mathrm{w}), 2203(\mathrm{~m}), 1670(\mathrm{~s})$, $1464(\mathrm{~m}), 1387(\mathrm{~m}), 1356(\mathrm{~m}), 1297(\mathrm{~m}), 1273(\mathrm{~m}), 1199(\mathrm{~m}), 1172(\mathrm{~m}), 1136$ (s), 1079 (s), 1060 (s), 1042 (s), 965 (m), 918 (s), 828 (s), 768 (m), 678 (m) cm ${ }^{-1} ;{ }^{1} \mathrm{H}$ NMR (600 $\left.\mathrm{MHz} \mathrm{CDCl}_{3}\right) \delta 9.18\left(\mathrm{~s}, 1 \mathrm{H}, \mathrm{C}_{1}-H\right), 4.30\left(\operatorname{app~qn}, J=6.4 \mathrm{~Hz}, 1 \mathrm{H}, \mathrm{C}_{4},-H\right), 4.15(\mathrm{dd}, J=$ 8.2, $6.1 \mathrm{~Hz}, 1 \mathrm{H}$, one of $\left.\mathrm{C}_{5},-H_{2}\right), 3.72\left(\mathrm{dd}, J=8.2,6.7 \mathrm{~Hz}, 1 \mathrm{H}\right.$, one of $\left.\mathrm{C}_{5},-H_{2}\right), 2.75(\mathrm{dd}, J$ $=17.4,5.3 \mathrm{~Hz}, 1 \mathrm{H}$, one of $\left.\mathrm{C}_{4}-H_{2}\right), 2.68\left(\mathrm{dd}, J=17.4,6.7 \mathrm{~Hz}, 1 \mathrm{H}\right.$, one of $\left.\mathrm{C}_{4}-H_{2}\right), 1.68(\mathrm{q}$, $\left.J=7.5 \mathrm{~Hz}, 2 \mathrm{H}, \mathrm{CH}_{2} \mathrm{CH}_{3}\right), 1.63\left(\mathrm{q}, J=7.5 \mathrm{~Hz}, 2 \mathrm{H}, \mathrm{CH}_{2} \mathrm{CH}_{3}\right), 0.92(\mathrm{t}, J=7.5 \mathrm{~Hz}, 3 \mathrm{H}$, $\left.\mathrm{CH}_{2} \mathrm{CH}_{3}\right), 0.90\left(\mathrm{t}, J=7.5 \mathrm{~Hz}, 3 \mathrm{H}, \mathrm{CH}_{2} \mathrm{CH}_{3}\right) ;{ }^{13} \mathrm{C} \mathrm{NMR}\left(150 \mathrm{MHz}, \mathrm{CDCl}_{3}\right) \delta 176.7\left(\mathrm{C}_{1}\right)$, $114.0\left(\mathrm{C}_{2}\right), 93.7\left(\mathrm{C}_{2}\right), 82.5\left(\mathrm{C}_{3}\right), 73.1\left(\mathrm{C}_{4}\right), 69.1\left(\mathrm{C}_{5}\right), 29.7\left(\mathrm{CH}_{2} \mathrm{CH}_{3}\right), 29.3\left(\mathrm{CH}_{2} \mathrm{CH}_{3}\right)$, $24.2\left(\mathrm{C}_{4}\right), 8.1\left(\mathrm{CH}_{2} \mathrm{CH}_{3}\right), 7.9\left(\mathrm{CH}_{2} \mathrm{CH}_{3}\right)$; high resolution mass spectrum (+ESI) $\mathrm{m} / \mathrm{z}$ 219.0997 [ $\mathrm{M}^{+}$; calcd for $\mathrm{C}_{11} \mathrm{H}_{16} \mathrm{O}_{3} \mathrm{Na}$ : 219.0997].

\section{4(R) 6-(2,2-Diethyl-[1,3]dioxolane-4-yl)-hexa-1,4-diyn-3-ol}

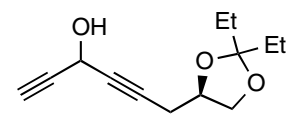

To a solution of 4-(2,2-diethyl-[1,3]dioxolane-4-yl)-but-2-ynal (7) (0.43g, $2.04 \mathrm{mmol})$ in tetrahydrofuran $(10 \mathrm{~mL})$ stirred at $-78{ }^{\circ} \mathrm{C}$ for $30 \mathrm{~min}$. was added a solution of ethynylmagnesium bromide $(0.5 \mathrm{M}$ in tetrahydrofuran) $(8.15 \mathrm{~mL}, 4.07 \mathrm{mmol})$. The reaction mixture was allowed to warm to $-30{ }^{\circ} \mathrm{C}$ and stirred for $30 \mathrm{~min}$. The reaction 
mixture was quenched with saturated solution of aqueous $\mathrm{NH}_{4} \mathrm{Cl}(10 \mathrm{~mL})$. The layers were separated, and the aqueous phase was extracted with diethyl ether $(3 \times 10 \mathrm{~mL})$. The combined organic extracts were washed with $\mathrm{H}_{2} \mathrm{O}(1 \times 10 \mathrm{~mL})$, and brine $(1 \times 10 \mathrm{~mL})$, dried over $\mathrm{MgSO}_{4}$, filtered and concentrated under reduced pressure. Gradient flash chromatography (Petroleum ether/diethyl ether, 100:0 $\rightarrow$ 90:10 $\rightarrow$ 80:20 $\rightarrow$ 60:40) afforded the bis-ynol $(0.43 \mathrm{~g}, 95 \%)$ as a clear pale yellow oil: TLC $\mathrm{R}_{\mathrm{f}}=0.56(40 \%$ $\mathrm{Et}_{2} \mathrm{O} /$ P.E.); $[\alpha]_{D}^{25}-43.0$ (c 1.31, $\mathrm{CHCl}_{3}$ ); IR (neat) 3287 (br, m), 2941 (s), 2973 (s), 2883 (m), 2233 (w), 2123 (w), 1463 (s), 1380 (m), 1356 (m), 1297 (s), 1199 (s), 1171 (s), 1137 (s), 1078 (s), 1023 (s), 965 (m), 915 (s), 808 (m), 767 (m), 733 (s) cm ${ }^{-1} ;{ }^{1} \mathrm{H}$ NMR (400 $\left.\mathrm{MHz} \mathrm{CDCl}_{3}\right) \delta 5.09\left(\mathrm{dd}, J=7.4,2.3 \mathrm{~Hz}, 1 \mathrm{H}, \mathrm{C}_{3}-H\right), 4.24\left(\operatorname{app} \mathrm{qn}, J=7.0 \mathrm{~Hz}, 1 \mathrm{H}, \mathrm{C}_{4}\right.$,$H), 4.13\left(\mathrm{dd}, J=8.1,6.1 \mathrm{~Hz}, 1 \mathrm{H}\right.$, one of $\left.\mathrm{C}_{5},-H_{2}\right), 3.73\left(\operatorname{app~t}, J=7.5 \mathrm{~Hz}, 1 \mathrm{H}\right.$, one of $\mathrm{C}_{5}$,$\left.H_{2}\right), 2.62\left(\mathrm{ddd}, J=16.7,4.8,2.0 \mathrm{~Hz}, 1 \mathrm{H}\right.$, one of $\left.\mathrm{C}_{6}-H_{2}\right), 2.54\left(\mathrm{~d}, J=2.3 \mathrm{~Hz}, 1 \mathrm{H}, \mathrm{C}_{1}-H\right)$, $2.50\left(\mathrm{ddd}, J=16.7,7.4,2.1 \mathrm{~Hz}, 1 \mathrm{H}\right.$, one of $\left.\mathrm{C}_{6}-H_{2}\right), 2.33\left(\mathrm{~d}, J=7.4 \mathrm{~Hz}, 1 \mathrm{H}, \mathrm{C}_{3} \mathrm{OH}\right), 1.67$ (q, $\left.J=7.5 \mathrm{~Hz}, 2 \mathrm{H}, \mathrm{CH}_{2} \mathrm{CH}_{3}\right), 1.62\left(\mathrm{q}, J=7.5 \mathrm{~Hz}, 2 \mathrm{H}, \mathrm{CH}_{2} \mathrm{CH}_{3}\right), 0.92(\mathrm{t}, J=7.5 \mathrm{~Hz}, 3 \mathrm{H}$, $\left.\mathrm{CH}_{2} \mathrm{CH}_{3}\right), 0.90\left(\mathrm{t}, J=7.5 \mathrm{~Hz}, 3 \mathrm{H}, \mathrm{CH}_{2} \mathrm{CH}_{3}\right) ;{ }^{13} \mathrm{C} \mathrm{NMR}\left(100 \mathrm{MHz}, \mathrm{CDCl}_{3}\right) \delta 113.7\left(\mathrm{C}_{2}\right)$, $81.13\left(\mathrm{C}_{4}\right), 81.11\left(\mathrm{C}^{\prime}{ }_{4}\right), 81.04\left(\mathrm{C}_{5}\right), 78.9\left(\mathrm{C}_{2}\right), 73.8\left(\mathrm{C}_{4}\right), 72.5\left(\mathrm{C}_{1}\right), 69.2\left(\mathrm{C}_{5}\right), 69.2\left(\mathrm{C}^{\prime}{ }_{5}\right)$, $52.1\left(\mathrm{C}_{3}\right), 29.8\left(\mathrm{CH}_{2} \mathrm{CH}_{3}\right), 29.5\left(\mathrm{CH}_{2} \mathrm{CH}_{3}\right), 23.6\left(\mathrm{C}_{6}\right), 23.6\left(\mathrm{C}_{6}^{\prime}\right), 8.1\left(\mathrm{CH}_{2} \mathrm{CH}_{3}\right), 8.0$ $\left(\mathrm{CH}_{2} \mathrm{CH}_{3}\right)$; high resolution mass spectrum $(+\mathrm{ESI}) \mathrm{m} / \mathrm{z} 245.1154\left[(\mathrm{M}+\mathrm{Na})^{+}\right.$; calcd for $\mathrm{C}_{13} \mathrm{H}_{18} \mathrm{O}_{3} \mathrm{Na}$ : 245.1154].

\section{4 (R) 6-(2,2-Diethyl-[1,3]dioxolane-4-yl)-hexa-1,4-diyn-3-one (8).}

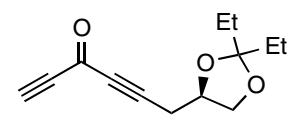

To a solution of 6-(2,2-diethyl-[1,3]dioxolane-4-yl)-hexa-1,4-diyn-3-ol (0.85 g, 3.84 $\mathrm{mmol})$ in dichloromethane $(20 \mathrm{~mL})$ stirred at $0{ }^{\circ} \mathrm{C}$ for $30 \mathrm{~min}$. was added Dess-Martin periodinane $(2.45 \mathrm{~g}, 5.77 \mathrm{mmol})$. The reaction mixture was allowed to warm to ambient temperature and stirred for $30 \mathrm{~min}$. The reaction mixture was quenched with a 1:1 solution of saturated aqueous $\mathrm{Na}_{2} \mathrm{~S}_{2} \mathrm{O}_{3}$ and saturated aqueous $\mathrm{NaHCO}_{3}(10 \mathrm{~mL})$. The layers were separated, and the aqueous phase was extracted with diethyl ether $(3 \times 20$ $\mathrm{mL})$. The combined organic extracts were washed with $\mathrm{H}_{2} \mathrm{O}(1 \times 20 \mathrm{~mL})$, and brine $(1 \times$ $20 \mathrm{~mL}$ ), dried over $\mathrm{MgSO}_{4}$, filtered and concentrated under reduced pressure. Gradient flash chromatography (Petroleum ether/diethyl ether, 100:0 $\rightarrow$ 90:10 $\rightarrow$ 80:20 $\rightarrow$ 60:40) afforded $8(0.82 \mathrm{~g}, 97 \%)$ as a clear yellow oil: TLC $\mathrm{R}_{\mathrm{f}}=0.61\left(50 \% \mathrm{Et}_{2} \mathrm{O} / \mathrm{P} . \mathrm{E}\right.$.); $[\alpha]_{D}^{25}$ 18.0 (c 0.05, $\mathrm{CHCl}_{3}$ ); IR (neat) 3250 (br, m), 2975 (s), 2940 (s), 2883 (s), 2225 (s), 2097 (s), 1633 (s), 1463 (s), 1379 (m), 1356 (m), 1273 (s), 1208 (s), 1172 (s), 1131 (s), 1079 (s), 1041 (s), 955 (s), 916 (s), 726 (s) cm $\left.{ }^{-1} ;{ }^{1} \mathrm{H} \mathrm{NMR} \mathrm{(600} \mathrm{MHz,} \mathrm{CDCl}{ }_{3}\right) \delta 4.30$ (m, $1 \mathrm{H}$, $\left.\mathrm{C}_{4},-H\right), 4.15\left(\mathrm{dd}, J=8.1,6.1 \mathrm{~Hz}, 1 \mathrm{H}\right.$, one of $\left.\mathrm{C}_{5},-H_{2}\right), 3.74(\mathrm{dd}, J=8.1,6.9 \mathrm{~Hz}, 1 \mathrm{H}$, one of $\left.\mathrm{C}_{5},-\mathrm{H}_{2}\right), 3.29\left(\mathrm{~s}, 1 \mathrm{H}, \mathrm{C}_{1}-H\right), 2.76\left(\mathrm{dd}, J=17.4,4.9 \mathrm{~Hz}, 1 \mathrm{H}\right.$, one of $\left.\mathrm{C}_{6}-H_{2}\right), 2.70(\mathrm{dd}, J$ $=17.4,6.7 \mathrm{~Hz}, 1 \mathrm{H}$, one of $\left.\mathrm{C}_{6}-\mathrm{H}_{2}\right), 1.68\left(\mathrm{q}, J=7.5 \mathrm{~Hz}, 2 \mathrm{H}, \mathrm{CH}_{2} \mathrm{CH}_{3}\right), 1.62(\mathrm{q}, J=7.5$ $\left.\mathrm{Hz}, 2 \mathrm{H}, \mathrm{CH}_{2} \mathrm{CH}_{3}\right), 0.91\left(\mathrm{t}, J=7.5 \mathrm{~Hz}, 3 \mathrm{H}, \mathrm{CH}_{2} \mathrm{CH}_{3}\right), 0.90\left(\mathrm{t}, J=7.5 \mathrm{~Hz}, 3 \mathrm{H}, \mathrm{CH}_{2} \mathrm{CH}_{3}\right)$; ${ }^{13} \mathrm{C}$ NMR $\left(150 \mathrm{MHz}, \mathrm{CDCl}_{3}\right) \delta 159.9\left(\mathrm{C}_{3}\right), 114.0\left(\mathrm{C}_{2}\right), 91.3\left(\mathrm{C}_{5}\right), 82.8\left(\mathrm{C}_{4}\right), 82.1\left(\mathrm{C}_{2}\right)$, $78.6\left(\mathrm{C}_{1}\right), 73.0\left(\mathrm{C}_{4}\right), 69.0\left(\mathrm{C}_{5}\right), 29.7\left(\mathrm{CH}_{2} \mathrm{CH}_{3}\right), 29.4\left(\mathrm{CH}_{2} \mathrm{CH}_{3}\right), 24.1\left(\mathrm{C}_{6}\right), 8.1\left(\mathrm{CH}_{2} \mathrm{CH}_{3}\right)$, 
$8.0\left(\mathrm{CH}_{2} \mathrm{CH}_{3}\right)$; high resolution mass spectrum (+ESI) $\mathrm{m} / z 243.0997\left[(\mathrm{M}+\mathrm{Na})^{+}\right.$; calcd for $\mathrm{C}_{13} \mathrm{H}_{16} \mathrm{O}_{3} \mathrm{Na}$ : 243.0997]; elemental analysis $\mathrm{C}, 70.74 \%$; $\mathrm{H}, 7.44 \%$.

$4(R)$ 1-([1,3]Dithian-2-yl)-5-([1,3]dithian-2-yl)-6(2,2-diethyl-[1,3]dioxolane-4-yl)hexa-3-one (9).

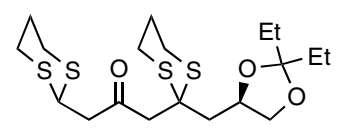

To a solution of 6-(2,2-diethyl-[1,3]dioxolane-4-yl)-hexa-1,4-diyn-3-one (8) (400 mg, $1.82 \mathrm{mmol})$ and propane-1,3-dithiol $(433 \mathrm{mg}, 4.00 \mathrm{mmol})$ in dichloromethane $(16 \mathrm{~mL})$ and methanol $(16 \mathrm{~mL})$ stirred at $-10^{\circ} \mathrm{C}$ for $30 \mathrm{~min}$. was added sodium methoxide (216 $\mathrm{mg}, 4.00 \mathrm{mmol})$. The reaction mixture was allowed to warm to ambient temperature over $17 \mathrm{~h}$. The reaction mixture was quenched with saturated aqueous $\mathrm{NH}_{4} \mathrm{Cl}$ solution $(20$ $\mathrm{mL}$ ). The layers were separated, and the aqueous phase was extracted with diethyl ether $(3 \times 20 \mathrm{~mL})$. The combined organic extracts were washed with $\mathrm{H}_{2} \mathrm{O}(1 \times 20 \mathrm{~mL})$, and brine $(1 \times 20 \mathrm{~mL})$, dried over $\mathrm{MgSO}_{4}$, filtered and concentrated under reduced pressure. Gradient flash chromatography (Petroleum ether/diethyl ether, 100:0 $\rightarrow$ 90:10 $\rightarrow$ 80:20 $\rightarrow 70: 30 \rightarrow 60: 40)$ afforded $9(0.68 \mathrm{~g}, 86 \%)$ as a clear pale yellow oil: TLC $_{\mathrm{f}}=0.40$ (50\% Et $\mathrm{Et}_{2}$ /P.E.); $[\alpha]_{D}^{25}+2.6$ (c 1.035, $\mathrm{CHCl}_{3}$ ); IR (neat) 2967 (s), 2932 (s), 2359 (w), 1723 (s), 1462 (s), 1422 (s), 1352 (s), 1276 (s), 1241 (m), 1194 (s), 1171 (s), 1077 (s), $912(\mathrm{~s}), 873(\mathrm{~m}), 765(\mathrm{~m}), 658(\mathrm{~m}) \mathrm{cm}^{-1} ;{ }^{1} \mathrm{H}$ NMR $\left(400 \mathrm{MHz}, \mathrm{CDCl}_{3}\right) \delta 4.49(\mathrm{t}, J=6.5$ $\left.\mathrm{Hz}, 1 \mathrm{H}, \mathrm{C}_{1}-H\right), 4.38\left(\mathrm{~m}, 1 \mathrm{H}, \mathrm{C}_{4}-H\right), 4.10\left(\mathrm{dd}, J=7.7,6.1 \mathrm{~Hz}, 1 \mathrm{H}\right.$, one of $\left.\mathrm{C}_{5},-H_{2}\right), 3.48$ $\left(\mathrm{t}, J=8.2 \mathrm{~Hz}, 1 \mathrm{H}\right.$, one of $\left.\mathrm{C}_{5}-H_{2}\right), 3.27\left(\mathrm{~d}, J=16.5 \mathrm{~Hz}, 1 \mathrm{H}\right.$, one of $\left.\mathrm{C}_{4}-H_{2}\right), 3.13(\mathrm{~d}, J=$ $16.5 \mathrm{~Hz}, 1 \mathrm{H}$, one of $\left.\mathrm{C}_{4}-\mathrm{H}_{2}\right), 2.86\left(\mathrm{~m}, 10 \mathrm{H}\right.$, both of $\mathrm{SCH}_{2} \mathrm{CH}_{2} \mathrm{CH}_{2} \mathrm{~S}$ and $\left.\mathrm{C}_{2}-\mathrm{H}_{2}\right), 2.54(\mathrm{dd}$, $J=14.9,8.1 \mathrm{~Hz}, 1 \mathrm{H}$, one of $\left.\mathrm{C}_{6}-H_{2}\right), 2.29\left(\mathrm{dd}, J=14.9,2.7 \mathrm{~Hz}, 1 \mathrm{H}\right.$, one of $\left.\mathrm{C}_{6}-H_{2}\right), 2.08$ $\left(\mathrm{m}, 1 \mathrm{H}\right.$, one of $\left.\mathrm{SCH}_{2} \mathrm{CH}_{2} \mathrm{CH}_{2} \mathrm{~S}\right), 1.97$ (app q, $\left.J=6.0 \mathrm{~Hz}, 2 \mathrm{H}, \mathrm{SCH}_{2} \mathrm{CH}_{2} \mathrm{CH}_{2} \mathrm{~S}\right), 1.85$ (m, $1 \mathrm{H}$, one of $\left.\mathrm{SCH}_{2} \mathrm{CH}_{2} \mathrm{CH}_{2} \mathrm{~S}\right), 1.59\left(\mathrm{~m}, 4 \mathrm{H}\right.$, both of $\left.\mathrm{CH}_{2} \mathrm{CH}_{3}\right), 0.88(\mathrm{t}, J=7.5 \mathrm{~Hz}, 3 \mathrm{H}$, $\left.\mathrm{CH}_{2} \mathrm{CH}_{3}\right), 0.86\left(\mathrm{t}, J=7.5 \mathrm{~Hz}, 3 \mathrm{H}, \mathrm{CH}_{2} \mathrm{CH}_{3}\right) ;{ }^{13} \mathrm{C} \mathrm{NMR}\left(100 \mathrm{MHz}, \mathrm{CDCl}_{3}\right) \delta 201.4\left(\mathrm{C}_{3}\right)$, $113.2\left(\mathrm{C}_{2}\right), 73.0\left(\mathrm{C}_{4}\right), 70.4\left(\mathrm{C}_{5}\right), 51.0\left(\mathrm{C}_{4}\right), 49.2\left(\mathrm{C}_{2}\right), 48.6\left(\mathrm{C}_{5}\right), 41.0\left(\mathrm{C}_{6}\right), 40.9\left(\mathrm{C}_{1}\right), 30.3$ $\left(\mathrm{SCH}_{2} \mathrm{CH}_{2} \mathrm{CH}_{2} \mathrm{~S}\right), 30.2\left(\mathrm{SCH}_{2} \mathrm{CH}_{2} \mathrm{CH}_{2} \mathrm{~S}\right), 30.0\left(\mathrm{CH}_{2} \mathrm{CH}_{3}\right), 30.0\left(\mathrm{CH}_{2} \mathrm{CH}_{3}\right), 26.4$ $\left(\mathrm{SCH}_{2} \mathrm{CH}_{2} \mathrm{CH}_{2} \mathrm{~S}\right), 26.4\left(\mathrm{SCH}_{2} \mathrm{CH}_{2} \mathrm{CH}_{2} \mathrm{~S}\right), 25.3\left(\mathrm{SCH}_{2} \mathrm{CH}_{2} \mathrm{CH}_{2} \mathrm{~S}\right), 24.8\left(\mathrm{SCH}_{2} \mathrm{CH}_{2} \mathrm{CH}_{2} \mathrm{~S}\right)$, $8.3\left(\mathrm{CH}_{2} \mathrm{CH}_{3}\right), 7.9\left(\mathrm{CH}_{2} \mathrm{CH}_{3}\right)$; high resolution mass spectrum (+ESI) $\mathrm{m} / \mathrm{z} 459.1132$ $\left[(\mathrm{M}+\mathrm{Na})^{+}\right.$; calcd for $\mathrm{C}_{19} \mathrm{H}_{32} \mathrm{O}_{3} \mathrm{~S}_{4} \mathrm{Na}$ : 459.1132]; elemental analysis $\mathrm{C}, 52.40 \% ; \mathrm{H}, 7.38 \%$.

\section{Dithiane (10)}
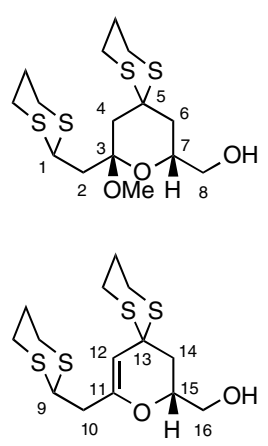
To a solution of 4 ( $R$ ) 1-([1,3]dithian-2-yl)-5-([1,3]dithian-2-yl)-6(2,2-diethyl[1,3]dioxolane-4-yl)-hexa-3-one (9). (50 mg, $0.11 \mathrm{mmol})$ in tetrahydrofuran $(0.5 \mathrm{~mL})$, trimethylorthoformate $(0.5 \mathrm{~mL})$ and methanol $(5 \mathrm{~mL})$ was added 10-camphorsulfonic acid $(40 \mathrm{mg}, 0.17 \mathrm{mmol})$. The reaction mixture was heated to $45^{\circ} \mathrm{C}$ for $4 \mathrm{~h}$. The reaction mixture was quenched with saturated aqueous $\mathrm{NaHCO}_{3}$ solution $(5 \mathrm{~mL})$. The layers were separated, and the aqueous phase was extracted with diethyl ether $(3 \times 5 \mathrm{~mL})$. The combined organic extracts were washed with $\mathrm{H}_{2} \mathrm{O}(1 \times 5 \mathrm{~mL})$, and brine $(1 \times 5 \mathrm{~mL})$, dried over $\mathrm{MgSO}_{4}$, filtered and concentrated under reduced pressure. Gradient flash chromatography (Petroleum ether/diethyl ether, 100:0 $\rightarrow$ 90:10 $\rightarrow$ 80:20 $\rightarrow$ 70:30 $\rightarrow$ $60: 40 \rightarrow 50: 50 \rightarrow 40: 60)$ afforded $\mathbf{1 0}(28.8 \mathrm{mg}, 70 \%$, or $83 \%$ by recovered starting material) an inseparable mixture of the desired methyl hemiketal and the enol ether as a white foam, and recovered $9(8.0 \mathrm{mg})$ as a clear pale yellow oil. In order to ascertain the ratio of enol ether to the methyl hemiketal, the mixture of products was silylated, and separated.

${ }^{1} \mathrm{H}$ NMR (600 MHz, $\left.\mathrm{CDCl}_{3}\right) \delta 6.96(\mathrm{~s}), 4.99(\mathrm{~s}), 4.80(\mathrm{~s}), 4.75(\mathrm{~s}), 4.25(\mathrm{~m}), 4.12(\mathrm{dd}, J=$ 4.1, $7.5 \mathrm{~Hz}), 4.03(\mathrm{~m}), 3.85(\mathrm{~m}), 3.72(\mathrm{~m}), 3.59(\mathrm{~m}), 3.32(\mathrm{~m}), 3.19(\mathrm{~s}), 3.08(\mathrm{dt}, J=2.6$, $14.1 \mathrm{~Hz}), 2.98(\mathrm{dt}, J=1.8,14.1 \mathrm{~Hz}), 2.93(\mathrm{~d}, J=1.9 \mathrm{~Hz}), 2.90(\mathrm{~d}, J=6.1 \mathrm{~Hz}), 2.86$ (tdd, $J=0.9,3.9,10.5 \mathrm{~Hz}), 2.80(\mathrm{dt}, J=3.3,10.5 \mathrm{~Hz}), 2.67(\mathrm{~m}), 2.56(\mathrm{~d}, J=13.8 \mathrm{~Hz}), 2.53(\mathrm{~d}$, $J=6.7 \mathrm{~Hz}), 2.50(\mathrm{t}, J=7.6 \mathrm{~Hz}), 2.47(\mathrm{~d}, J=7.6 \mathrm{~Hz}), 2.41(\mathrm{~d}, J=14.2 \mathrm{~Hz}), 2.26(\mathrm{~s}), 2.10$ (m), 1.96 (dd, $J=7.7,15.2 \mathrm{~Hz}), 1.84(\mathrm{~m}), 1.65(\mathrm{~s}), 1.42(\mathrm{~s}), 1.24(\mathrm{~s}) ;{ }^{13} \mathrm{C}$ NMR $(150$ $\left.\mathrm{MHz}, \mathrm{CDCl}_{3}\right) \delta$ 152.2, 125.5, 102.5, 99.6, 74.5, 68.6, 65.2, 64.6, 53.4, 47.7, 46.6, 44.9, $42.7,41.7,39.9,37.3,31.2,30.8,30.4,30.3,29.6,27.3,27.0,26.5,26.3,25.7,25.3,25.2$, 24.5; high resolution mass spectrum (+ESI) $\mathrm{m} / \mathrm{z} 373.0400\left[(\mathrm{M}+\mathrm{Na})^{+}\right.$; calcd for $\mathrm{C}_{14} \mathrm{H}_{22} \mathrm{O}_{2} \mathrm{~S}_{4} \mathrm{Na}$ : 373.0400$] \mathrm{m} / z 405.0663$ [(M+Na) ${ }^{+}$; calcd for $\left.\mathrm{C}_{15} \mathrm{H}_{26} \mathrm{O}_{3} \mathrm{~S}_{4} \mathrm{Na}: 405.0662\right]$.

\section{Silylated Dithiane}

To a solution of $\mathbf{1 0}(28.8 \mathrm{mg}$, as a mixture of pyrans) in tetrahydrofuran $(2.0 \mathrm{~mL})$, was added imidazole (14 mg, $0.21 \mathrm{mmol})$ and TBSCl $(16 \mathrm{mg}, 0.10 \mathrm{mg})$. The reaction mixture was stirred at ambient temperature for $16 \mathrm{~h}$. The reaction mixture was poured through a fritted tube to remove excess salt and concentrated under reduced pressure. Gradient flash chromatography (Petroleum ether/diethyl ether, 100:0 $\rightarrow$ 99:1 $\rightarrow$ 98:2 $\rightarrow$ 97:3 $\rightarrow$ 96:4 $\rightarrow$ 95:5 $\rightarrow$ 94:6 $\rightarrow$ 92:8 $\rightarrow$ 90:10) afforded TBS protected enol ether $(26.2 \mathrm{mg})$ and TBS protected methyl hemiketal $(10.3 \mathrm{mg})$ as yellow oils.

\section{TBS protected enol ether}

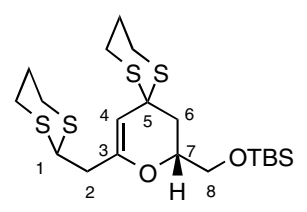

TLC $\mathrm{R}_{\mathrm{f}}=0.24\left(10 \% \mathrm{Et}_{2} \mathrm{O} / \mathrm{P} . \mathrm{E}\right.$.); ${ }^{1} \mathrm{H}$ NMR $\left(600 \mathrm{MHz}, \mathrm{CDCl}_{3}\right) \delta 4.78\left(\mathrm{~s}, 1 \mathrm{H}, \mathrm{C}_{4}-H\right), 4.25$ $\left(\mathrm{t}, J=7.4 \mathrm{~Hz}, 1 \mathrm{H}, \mathrm{C}_{1}-H\right), 4.17\left(\mathrm{~m}, 1 \mathrm{H}, \mathrm{C}_{7}-H\right), 3.81\left(\mathrm{dd}, J=4.7,10.6 \mathrm{~Hz}, 1 \mathrm{H}\right.$, one of $\mathrm{C}_{8}{ }^{-}$ 
$\left.H_{2}\right), 3.75\left(\mathrm{dd}, J=4.8,10.6 \mathrm{~Hz}, 1 \mathrm{H}\right.$, one of $\left.\mathrm{C}_{8}-\mathrm{H}_{2}\right), 2.98\left(\mathrm{~m}, 2 \mathrm{H}, \mathrm{SCH}_{2} \mathrm{CH}_{2} \mathrm{CH}_{2} \mathrm{~S}\right), 2.81$ $\left(\mathrm{m}, 5 \mathrm{H}, \mathrm{SCH}_{2} \mathrm{CH}_{2} \mathrm{CH}_{2} \mathrm{~S}\right), 2.69\left(\mathrm{~d}, J=13.7 \mathrm{~Hz}, 1 \mathrm{H}\right.$, one of $\left.\mathrm{C}_{6}-H_{2}\right), 2.64(\mathrm{~d}, J=13.7 \mathrm{~Hz}, 1$ $\mathrm{H}$, one of $\left.\mathrm{C}_{6}-H_{2}\right), 2.49\left(\mathrm{dd}, J=6.9,13.9 \mathrm{~Hz}, 1 \mathrm{H}\right.$, one of $\left.\mathrm{C}_{2}-H_{2}\right), 2.43(\mathrm{dd}, J=8.0,13.9$ $\mathrm{Hz}, 1 \mathrm{H}$, one of $\left.\mathrm{C}_{2}-\mathrm{H}_{2}\right), 2.07\left(\mathrm{~m}, 2 \mathrm{H}, \mathrm{SCH}_{2} \mathrm{CH}_{2} \mathrm{CH}_{2} \mathrm{~S}\right), 1.97$ (app t, $J=12.4 \mathrm{~Hz}, 1 \mathrm{H}$, one of $\left.\mathrm{SCH}_{2} \mathrm{CH}_{2} \mathrm{CH}_{2} \mathrm{~S}\right), 1.85\left(\mathrm{~m}, 2 \mathrm{H}, \mathrm{SCH}_{2} \mathrm{CH}_{2} \mathrm{CH}_{2} \mathrm{~S}\right), 0.91\left(\mathrm{~s}, 9 \mathrm{H}, \mathrm{OSi}\left(\mathrm{CH}_{3}\right)_{2} \mathrm{C}\left(\mathrm{CH}_{3}\right)_{3}\right), 0.09$ (s, $\left.6 \mathrm{H}, \mathrm{OSi}\left(\mathrm{CH}_{3}\right)_{2} \mathrm{C}\left(\mathrm{CH}_{3}\right)_{3}\right) ;{ }^{13} \mathrm{C} \mathrm{NMR}\left(150 \mathrm{MHz}, \mathrm{CDCl}_{3}\right) \delta 152.3\left(\mathrm{C}_{3}\right), 102.3\left(\mathrm{C}_{4}\right), 74.5$ $\left(\mathrm{C}_{7}\right), 64.9\left(\mathrm{C}_{8}\right), 45.2\left(\mathrm{C}_{5}\right), 44.0\left(\mathrm{C}_{1}\right), 40.0\left(\mathrm{C}_{2}\right), 37.9\left(\mathrm{C}_{6}\right), 30.1\left(\mathrm{SCH}_{2} \mathrm{CH}_{2} \mathrm{CH}_{2} \mathrm{~S}\right), 30.0$

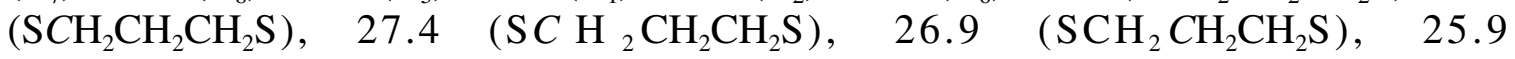
$\left(\mathrm{OSi}\left(\mathrm{CH}_{3}\right)_{2} \mathrm{C}\left(\mathrm{CH}_{3}\right)_{3}\right), \quad 25.8 \quad\left(\mathrm{SCH}_{2} \mathrm{CH}_{2} \mathrm{CH}_{2} \mathrm{~S}\right), \quad 24.6 \quad\left(\mathrm{SCH}_{2} \mathrm{CH}_{2} \mathrm{CH}_{2} \mathrm{~S}\right), \quad-3.6$ $\left(\mathrm{OSi}\left(\mathrm{CH}_{3}\right)_{2} \mathrm{C}\left(\mathrm{CH}_{3}\right)_{3}\right),-5.3\left(\mathrm{OSi}\left(\mathrm{CH}_{3}\right)_{2} \mathrm{C}\left(\mathrm{CH}_{3}\right)_{3}\right)$.

\section{TBS protected methyl hemiketal}

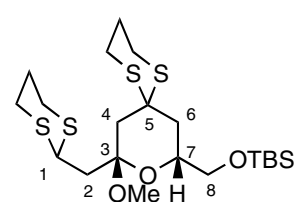

TLC $\mathrm{R}_{\mathrm{f}}=0.12\left(10 \% \mathrm{Et}_{2} \mathrm{O} / \mathrm{P} . \mathrm{E}\right.$.); ${ }^{1} \mathrm{H}$ NMR $\left(600 \mathrm{MHz}, \mathrm{CDCl}_{3}\right) \delta 4.12(\mathrm{dd}, J=4.5,6.7 \mathrm{~Hz}$, $\left.1 \mathrm{H}, \mathrm{C}_{1}-H\right), 3.98\left(\mathrm{~m}, 1 \mathrm{H}, \mathrm{C}_{7}-H\right), 3.71\left(\mathrm{dd}, \mathrm{J}=5.6,10.5 \mathrm{~Hz}, 1 \mathrm{H}\right.$, one of $\left.\mathrm{C}_{8}-H_{2}\right), 3.60(\mathrm{dd}$, $J=5.3,10.5 \mathrm{~Hz}, 1 \mathrm{H}$, one of $\left.\mathrm{C}_{8}-H_{2}\right), 3.19\left(\mathrm{~s}, 3 \mathrm{H}, \mathrm{C}_{3} \mathrm{OCH}_{3}\right), 3.09(\mathrm{~m}, 1 \mathrm{H}$, $\left.\mathrm{SCH}_{2} \mathrm{CH}_{2} \mathrm{CH}_{2} \mathrm{~S}\right), 2.91\left(\mathrm{~m}, 3 \mathrm{H}, \mathrm{SCH}_{2} \mathrm{CH}_{2} \mathrm{CH}_{2} \mathrm{~S}\right), 2.77\left(\mathrm{~m}, 2 \mathrm{H}, \mathrm{SCH}_{2} \mathrm{CH}_{2} \mathrm{CH}_{2} \mathrm{~S}\right), 2.70(\mathrm{~m}$, $1 \mathrm{H}, \mathrm{SCH}_{2} \mathrm{CH}_{2} \mathrm{CH}_{2} \mathrm{~S}$ ), 2.57 (app d, $J=13.7 \mathrm{~Hz}, 2 \mathrm{H}, \mathrm{C}_{4}-H_{2}$ ), 2.13 (dd, $J=4.3,15.5 \mathrm{~Hz}, 1$ $\mathrm{H}$, one of $\left.\mathrm{C}_{2}-\mathrm{H}_{2}\right), 2.06\left(\mathrm{~m}, 1 \mathrm{H}, \mathrm{SCH}_{2} \mathrm{CH}_{2} \mathrm{CH}_{2} \mathrm{~S}\right), 1.84\left(\mathrm{~m}, 3 \mathrm{H}, \mathrm{SCH}_{2} \mathrm{CH}_{2} \mathrm{CH}_{2} \mathrm{~S}\right.$ and one of $\left.\mathrm{C}_{2}-\mathrm{H}_{2}\right), 1.63\left(\mathrm{~m}, 2 \mathrm{H}, \mathrm{SCH}_{2} \mathrm{CH}_{2} \mathrm{CH}_{2} \mathrm{~S}\right), 0.90$ (s, $\left.9 \mathrm{H}, \mathrm{OSi}\left(\mathrm{CH}_{3}\right)_{2} \mathrm{C}\left(\mathrm{CH}_{3}\right)_{3}\right), 0.07$ (s, $6 \mathrm{H}$, $\left.\mathrm{OSi}\left(\mathrm{CH}_{3}\right)_{2} \mathrm{C}\left(\mathrm{CH}_{3}\right)_{3}\right) ;{ }^{13} \mathrm{C} \mathrm{NMR}\left(150 \mathrm{MHz}, \mathrm{CDCl}_{3}\right) \delta 99.5\left(\mathrm{C}_{3}\right), 68.7\left(\mathrm{C}_{7}\right), 65.7\left(\mathrm{C}_{8}\right), 47.6$ $\left(\mathrm{C}_{3} \mathrm{OCH}_{3}\right), 46.8\left(\mathrm{C}_{5}\right), 45.7\left(\mathrm{C}_{1}\right), 42.9\left(\mathrm{C}_{2}\right), 38.2\left(\mathrm{C}_{4}\right), 31.2\left(\mathrm{SCH}_{2} \mathrm{CH}_{2} \mathrm{CH}_{2} \mathrm{~S}\right), 30.9$ $\left(\mathrm{SCH}_{2} \mathrm{CH}_{2} \mathrm{CH}_{2} \mathrm{~S}\right), 30.3\left(\mathrm{C}_{6}\right), 29.7 \quad\left(\mathrm{SCH}_{2} \mathrm{CH}_{2} \mathrm{CH}_{2} \mathrm{~S}\right), 26.5 \quad\left(\mathrm{SCH}_{2} \mathrm{CH}_{2} \mathrm{CH}_{2} \mathrm{~S}\right), 25.9$ $\left(\begin{array}{llllll}\left(\mathrm{OSi}\left(\mathrm{CH}_{3}\right)_{2} \mathrm{C}\left(\mathrm{CH}_{3}\right)_{3}\right), & 25.3 & \left(\mathrm{SCH}_{2} \mathrm{CH}_{2} \mathrm{CH}_{2} \mathrm{~S}\right), & 18.3 & \left(\mathrm{SCH}_{2} \mathrm{CH}_{2} \mathrm{CH}_{2} \mathrm{~S}\right) & 1.0\end{array}\right.$ $\left(\mathrm{OSi}\left(\mathrm{CH}_{3}\right)_{2} \mathrm{C}\left(\mathrm{CH}_{3}\right)_{3}\right),-5.3\left(\mathrm{OSi}\left(\mathrm{CH}_{3}\right)_{2} \mathrm{C}\left(\mathrm{CH}_{3}\right)_{3}\right)$.

2(R), 3(S), 4(R), 2,4-Dimethyl-hex-5-ene-1,3-diol (11).

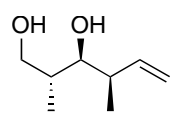

A solution of tetrabutylammonium fluoride (TBAF) (1.0 M in THF) (39.20 mL, 39.20 mmol) was added dropwise to a solution of $2(R), 3(S), 1$-(tert-butyldiphenylsilanyloxy)2,4-dimethyl-3-hydroxy-5-hexene (10 $00 \mathrm{~g}, 26.14 \mathrm{mmol})$ in anhydrous tetrahydrofuran $(250 \mathrm{~mL})$. The colorless mixture was then stirred at ambient temperature for $1 \mathrm{~h}$ at which time a solution of TBAF (1.0 M in THF) $(13.07 \mathrm{~mL}, 13.07 \mathrm{mmol})$ was added and the reaction mixture was stirred at ambient temperature for $20 \mathrm{~min}$. The reaction was quenched with saturated aqueous $\mathrm{NH}_{4} \mathrm{Cl}$ solution $(100 \mathrm{~mL})$. The layers were separated, and the aqueous phase was extracted with diethyl ether $(3 \times 100 \mathrm{~mL})$. The combined organic extracts were dried over $\mathrm{MgSO}_{4}$, filtered and concentrated under reduced pressure. Gradient flash chromatography (Petroleum ether/ diethyl ether, 100:0 $\rightarrow$ 75:25 
$\rightarrow$ 50:50 $\rightarrow$ 40:60 $\rightarrow$ 25:75) afforded $\mathbf{1 1}(3.60 \mathrm{~g}, 95 \%)$ as a clear pale yellow oil: TLC $\mathrm{R}_{\mathrm{f}}$ $=0.12\left(50 \% \mathrm{Et}_{2} \mathrm{O} / \mathrm{P} . \mathrm{E}\right.$.) $;[\alpha]_{D}^{25}+11.5\left(c 1.05, \mathrm{CHCl}_{3}\right)$; IR (neat) 3339 (br, s), $2965(\mathrm{~s})$, 2878 (s), 1640 (s), 1456 (s), 1420 (s), 1377 (s), 1069 (s), 1027 (s), 996 (s), 969 (s), 911 (s), $859(\mathrm{w}), 733(\mathrm{~s}), 679(\mathrm{~s}) \mathrm{cm}^{-1} ;{ }^{1} \mathrm{H} \mathrm{NMR}\left(400 \mathrm{MHz}, \mathrm{CDCl}_{3}\right) \delta 5.85$ (ddd, $J=17.2$, $\left.10.8,6.5 \mathrm{~Hz}, 1 \mathrm{H}, \mathrm{C}_{5}-\mathrm{H}\right), 5.14\left(\mathrm{~s}, 1 \mathrm{H}\right.$, one of $\left.\mathrm{C}_{6}-H_{2}\right), 5.09\left(\mathrm{~d}, J=1.3 \mathrm{~Hz}, 1 \mathrm{H}\right.$, one of $\mathrm{C}_{6}$ $\left.H_{2}\right), 3.75\left(\mathrm{dd}, J=10.8,3.5 \mathrm{~Hz}, 1 \mathrm{H}\right.$, one of $\left.\mathrm{C}_{1}-H_{2}\right), 3.64(\mathrm{dd}, J=10.8,7.1 \mathrm{~Hz}, 1 \mathrm{H}$, one of $\left.\mathrm{C}_{1}-H_{2}\right), 3.48\left(\mathrm{dd}, J=7.9,3.9 \mathrm{~Hz}, 1 \mathrm{H}, \mathrm{C}_{3}-H\right), 2.45\left(\mathrm{~m}, 1 \mathrm{H}, \mathrm{C}_{4}-H\right), 1.85\left(\mathrm{~m}, 1 \mathrm{H}, \mathrm{C}_{2}-H\right)$, $1.03\left(\mathrm{~d}, J=6.9 \mathrm{~Hz}, 3 \mathrm{H}, \mathrm{C}_{4} \mathrm{CH}_{3}\right), 0.90\left(\mathrm{~d}, J=7.0 \mathrm{~Hz}, 3 \mathrm{H}, \mathrm{C}_{2} \mathrm{CH}_{3}\right) ;{ }^{13} \mathrm{C} \mathrm{NMR}(100 \mathrm{MHz}$, $\left.\mathrm{CDCl}_{3}\right) \delta 141.5\left(\mathrm{C}_{5}\right), 115.3\left(\mathrm{C}_{6}\right), 79.6\left(\mathrm{C}_{3}\right), 67.9\left(\mathrm{C}_{1}\right), 40.1\left(\mathrm{C}_{4}\right), 36.9\left(\mathrm{C}_{2}\right), 13.8\left(\mathrm{C}_{2} \mathrm{CH}_{3}\right)$, $11.7\left(\mathrm{C}_{4} \mathrm{CH}_{3}\right)$; high resolution mass spectrum (+ESI) $\mathrm{m} / z$ 167.1048 $\left[(\mathrm{M}+\mathrm{Na})^{+}\right.$; calcd for $\mathrm{C}_{8} \mathrm{H}_{16} \mathrm{O}_{2} \mathrm{Na}:$ 167.1048].

\section{Paramethoxybenzylidene acetal (17)}

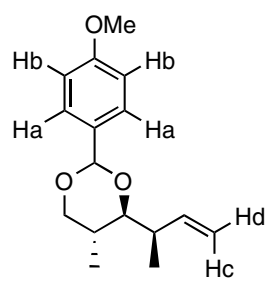

Under argon, a solution of 2(R), 3(S), 4(R), 2,4-dimethyl-hex-5-ene-1,3-diol (11) (3.46 g, $23.99 \mathrm{mmol})$ and $p$-anisaldehyde dimethyl acetal $(21.86 \mathrm{~g}, 119.96 \mathrm{mmol})$ in anhydrous chloroform $(200 \mathrm{~mL})$ was treated with $p$-TsOH $(456 \mathrm{mg}, 2.40 \mathrm{mmol})$. The reaction mixture was stirred at ambient temperature for $1 \mathrm{~h} 25 \mathrm{~min}$. The pale pink reaction mixture was quenched with saturated aqueous $\mathrm{NaHCO}_{3}$ solution $(100 \mathrm{~mL})$. The layers were separated, and the aqueous phase was extracted with diethyl ether $(3 \times 100 \mathrm{~mL})$. The combined organic extracts were dried over $\mathrm{MgSO}_{4}$, filtered and concentrated under reduced pressure. Gradient flash chromatography (Petroleum ether/diethyl ether, 100:0 $\rightarrow$ 90:10 $\rightarrow$ 80:20 $\rightarrow$ 70:30 $\rightarrow$ 60:40) afforded the paramethoxybenzylidene acetal (5.73 $\mathrm{g}, 91 \%)$ as a clear pale yellow oil: TLC $\mathrm{R}_{\mathrm{f}}=0.87\left(50 \% \mathrm{Et}_{2} \mathrm{O} / \mathrm{P} . \mathrm{E}\right.$.); $[\alpha]_{D}^{25}-67.2(c 1.025$, $\mathrm{CHCl}_{3}$ ); IR (neat) 2934 (s), 2965 (s), 2836 (s), 1615 (s), 1588 (w), 1517 (s), 1460 (s), 1419 (w), 1391 (s), 1370 (s), 1302 (s), 1247 (s), 1170 (s), 1147 (s), 1119 (s), 1077 (s), 1032 (s), 981 (s), 958 (s), 912 (s), 825 (s), 782 (s), 666 (s) cm ${ }^{-1} ;{ }^{1} \mathrm{H}$ NMR (600 MHz, $\left.\mathrm{CDCl}_{3}\right) \delta 7.42\left(\mathrm{~d}, J=8.7 \mathrm{~Hz}, 2 \mathrm{H}, \mathrm{Ar}-H_{2}, \mathrm{H}_{\mathrm{a}}\right), 6.89\left(\mathrm{~d}, J=8.7 \mathrm{~Hz}, 2 \mathrm{H}, \mathrm{Ar}-\mathrm{H}_{2}, \mathrm{H}_{\mathrm{b}}\right), 6.03$ (ddd, $\left.J=17.2,10.3,7.6 \mathrm{~Hz}, 1 \mathrm{H}, \mathrm{C}_{5}-H\right), 5.44(\mathrm{~s}, 1 \mathrm{H}, \mathrm{CHAr}), 5.08(\mathrm{~d}, J=17.2 \mathrm{~Hz}, 1 \mathrm{H}$, $\left.\mathrm{C}_{6}-H_{2}, \mathrm{H}_{\mathrm{c}}\right), 5.01\left(\mathrm{~d}, J=10.3 \mathrm{~Hz}, 1 \mathrm{H}, \mathrm{C}_{6}-H_{2}, \mathrm{H}_{\mathrm{d}}\right), 4.10(\mathrm{dd}, J=11.0,4.7 \mathrm{~Hz}, 1 \mathrm{H}$, one of $\left.\mathrm{C}_{1}-H_{2}\right), 3.80\left(\mathrm{~s}, 3 \mathrm{H}, \mathrm{ArOCH}_{3}\right), 3.50\left(\mathrm{t}, J=11.0 \mathrm{~Hz}, 1 \mathrm{H}\right.$, one of $\left.\mathrm{C}_{1}-H_{2}\right), 3.45(\mathrm{dd}, J=10.0$, $\left.2.4 \mathrm{~Hz}, 1 \mathrm{H}, \mathrm{C}_{3} H\right), 2.50\left(\mathrm{~m}, 1 \mathrm{H}, \mathrm{C}_{4}-H\right), 2.04\left(\mathrm{~m}, 1 \mathrm{H}, \mathrm{C}_{2}-H\right), 1.11(\mathrm{~d}, J=6.9 \mathrm{~Hz}, 3 \mathrm{H}$, $\left.\mathrm{C}_{4} \mathrm{CH}_{3}\right), 0.80\left(\mathrm{~d}, J=6.7 \mathrm{~Hz}, 3 \mathrm{H}, \mathrm{C}_{2} \mathrm{CH}_{3}\right) ;{ }^{13} \mathrm{C}$ NMR $\left(150 \mathrm{MHz}, \mathrm{CDCl}_{3}\right) \delta 159.8$ (Ar, $C$ $\left.\mathrm{OCH}_{3}\right), 142.4\left(\mathrm{C}_{5}\right), 131.5(\mathrm{Ar}), 127.3\left(\mathrm{Ar}, C-\mathrm{H}_{\mathrm{a}}\right), 113.6\left(\mathrm{C}_{6}\right), 113.5\left(\mathrm{Ar}, C-\mathrm{H}_{\mathrm{b}}\right), 100.9$ (CHAr), $86.0\left(\mathrm{C}_{3}\right), 73.1\left(\mathrm{C}_{1}\right), 55.3\left(\mathrm{ArOCH}_{3}\right), 39.0\left(\mathrm{C}_{4}\right), 30.9\left(\mathrm{C}_{2}\right), 13.2\left(\mathrm{C}_{4} \mathrm{CH}_{3}\right), 12.2$ $\left(\mathrm{C}_{2} \mathrm{CH}_{3}\right)$; high resolution mass spectrum $(+\mathrm{ESI}) \mathrm{m} / \mathrm{z} 285.1467\left[(\mathrm{M}+\mathrm{Na})^{+}\right.$; calcd for $\mathrm{C}_{16} \mathrm{H}_{22} \mathrm{O}_{3} \mathrm{Na}$ : 285.1467].

2(R), 3(S), 4(R), 3-(4'-Methoxy-benzyloxy)-2,4-dimethyl-hex-5-en-1-ol 


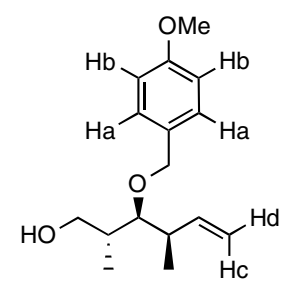

Under argon, a solution of $17(2.00 \mathrm{~g}, 7.62 \mathrm{mmol})$, in dichloromethane (100 mL) at -10 ${ }^{\circ} \mathrm{C}$ was treated with a solution of DIBAL-H $\left(1 \mathrm{M}\right.$ in $\left.\mathrm{CH}_{2} \mathrm{Cl}_{2}\right)(19.06 \mathrm{~mL}, 19.06 \mathrm{mmol})$. The reaction mixture was stirred at $-10^{\circ} \mathrm{C}$ for $2 \mathrm{~h}$. The reaction mixture was quenched with a 1:1 solution of methanol and toluene $(20 \mathrm{~mL})$ and poured into saturated Rochelle's salt (sodium potassium tartrate) solution and stirred for $1 \mathrm{~h}$. The layers were separated, and the aqueous phase was extracted with diethyl ether $(4 \times 100 \mathrm{~mL})$. The combined organic extracts were washed with $1 \mathrm{M} \mathrm{HCl}(1 \times 100 \mathrm{~mL}), \mathrm{H}_{2} \mathrm{O}(1 \times 100 \mathrm{~mL})$, and brine $(1 \times 100 \mathrm{~mL})$, dried over $\mathrm{MgSO}_{4}$, filtered and concentrated under reduced pressure. Gradient flash chromatography (Petroleum ether/diethyl ether, 100:0 $\rightarrow$ 90:10 $\rightarrow$ 80:20 $\rightarrow 70: 30 \rightarrow 60: 40 \rightarrow 50: 50 \rightarrow 40: 60)$ afforded the alcohol (4.81 g, 87\%) as a clear pale yellow oil: TLC $\mathrm{R}_{\mathrm{f}}=0.30\left(50 \% \mathrm{Et}_{2} \mathrm{O} / \mathrm{P} . \mathrm{E}.\right) ;[\alpha]_{D}^{25}+34.1\left(c 1.055, \mathrm{CHCl}_{3}\right)$; IR (neat) 3426 (m, br), 2964 (s), 2874 (s), 1638 (m), 1613 (s), 1586 (m), 1513 (s), 1456 (s), 1420 (m), 1347 (m), 1301 (s), 1246 (s), 1173 (s), 1033 (s), 951 (s), 913 (s), 821 (s), 757 (s), 682 (s) $\mathrm{cm}^{-1} ;{ }^{1} \mathrm{H}$ NMR $\left(400 \mathrm{MHz}, \mathrm{CDCl}_{3}\right) \delta 7.26\left(\mathrm{~d}, J=8.6 \mathrm{~Hz}, 2 \mathrm{H}, \mathrm{Ar}-H_{2}, \mathrm{H}_{\mathrm{a}}\right), 6.87(\mathrm{~d}, J=8,6$ $\mathrm{Hz}, 2 \mathrm{H}, \mathrm{Ar}-H_{2}, \mathrm{H}_{\mathrm{b}}$ ), 5.90 (ddd, $\left.J=17.3,10.3,7.4 \mathrm{~Hz}, 1 \mathrm{H}, \mathrm{C}_{5}-H\right), 5.09$ (d, $J=17.3 \mathrm{~Hz}, 1$ $\left.\mathrm{H}, \mathrm{C}_{6}-H_{2}, \mathrm{H}_{\mathrm{c}}\right), 5.04\left(\mathrm{~d}, J=10.3 \mathrm{~Hz}, 1 \mathrm{H}, \mathrm{C}_{6}-H_{2}, \mathrm{H}_{\mathrm{d}}\right), 4.60(\mathrm{~d}, J=10.6 \mathrm{~Hz}, 1 \mathrm{H}$, one of $\left.\mathrm{CH}_{2} \mathrm{Ar}\right), 4.47\left(\mathrm{~d}, \mathrm{~J}=10.6 \mathrm{~Hz}, 1 \mathrm{H}\right.$, one of $\left.\mathrm{CH}_{2} \mathrm{Ar}\right), 3.80\left(\mathrm{~s}, 3 \mathrm{H}, \mathrm{ArOCH}_{3}\right), 3.70(\mathrm{~m}, 1 \mathrm{H}$, one of $\left.\mathrm{C}_{1}-H_{2}\right), 3.58\left(\mathrm{~m}, 1 \mathrm{H}\right.$, one of $\left.\mathrm{C}_{1}-H_{2}\right), 3.30\left(\mathrm{dd}, J=5.7,5.7 \mathrm{~Hz}, 1 \mathrm{H}, \mathrm{C}_{3}-H\right), 2.80$ $\left(\mathrm{dd}, J=5.5 \mathrm{~Hz}, 5.5 \mathrm{~Hz}, 1 \mathrm{H}, \mathrm{C}_{1} \mathrm{OH}\right), 2.53\left(\mathrm{~m}, 1 \mathrm{H}, \mathrm{C}_{4}-H\right), 1.90\left(\mathrm{~m}, 1 \mathrm{H}, \mathrm{C}_{2}-H\right), 1.11(\mathrm{~d}, J$ $\left.=6.8 \mathrm{~Hz}, 3 \mathrm{H}, \mathrm{C}_{4} \mathrm{CH}_{3}\right), 1.00\left(\mathrm{~d}, J=7.1 \mathrm{~Hz}, 3 \mathrm{H}, \mathrm{C}_{2} \mathrm{CH}_{3}\right) ;{ }^{13} \mathrm{C} \mathrm{NMR}\left(100 \mathrm{MHz}, \mathrm{CDCl}_{3}\right)$ $\delta 159.3\left(\mathrm{Ar}, C-\mathrm{OCH}_{3}\right), 142.0\left(\mathrm{C}_{5}\right), 130.3(\mathrm{Ar}), 129.5\left(\mathrm{Ar}, C-\mathrm{H}_{\mathrm{a}}\right), 114.3\left(\mathrm{C}_{6}\right), 113.8(\mathrm{Ar}, C-$ $\left.\mathrm{H}_{\mathrm{b}}\right), 88.3\left(\mathrm{C}_{3}\right), 74.7\left(\mathrm{CH}_{2} \mathrm{Ar}\right), 66.0\left(\mathrm{C}_{1}\right), 55.2\left(\mathrm{ArOCH}_{3}\right), 40.8\left(\mathrm{C}_{4}\right), 37.2\left(\mathrm{C}_{2}\right), 15.5$ $\left(\mathrm{C}_{2} \mathrm{CH}_{3}\right)$, $14.6\left(\mathrm{C}_{4} \mathrm{CH}_{3}\right)$; high resolution mass spectrum (+ESI) $\mathrm{m} / \mathrm{z} 287.1623\left[(\mathrm{M}+\mathrm{Na})^{+}\right.$; calcd for $\mathrm{C}_{16} \mathrm{H}_{24} \mathrm{O}_{3} \mathrm{Na}$ : 287.1623].

2(R), 3(S), 4(R), 3-(4'-Methoxy-benzyloxy)-2,4-dimethyl-hex-5-enal

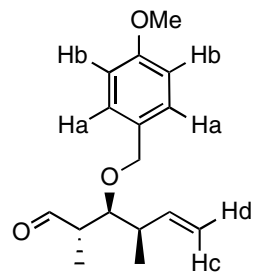

Under argon, a solution of 2(R), 3(S), 4(R), 3-(4-methoxy-benzyloxy)-2,4-dimethyl-hex5-en-1-ol $(1.40 \mathrm{~g}, 5.28 \mathrm{mmol})$, in dichloromethane $(50 \mathrm{~mL})$ at $0{ }^{\circ} \mathrm{C}$ was treated with Dess-Martin Periodinane $(3.36 \mathrm{~g}, 7.92 \mathrm{mmol})$. The reaction mixture was stirred at ambient temperature for $2 \mathrm{~h}$. The reaction mixture was quenched with a 1:1 solution of saturated aqueous $\mathrm{Na}_{2} \mathrm{~S}_{2} \mathrm{O}_{3}$ and saturated aqueous $\mathrm{NaHCO}_{3}(10 \mathrm{~mL})$. The layers were 
separated, and the aqueous phase was extracted with diethyl ether $(3 \times 50 \mathrm{~mL})$. The combined organic extracts were washed with $\mathrm{H}_{2} \mathrm{O}(1 \times 50 \mathrm{~mL})$, and brine $(1 \times 50 \mathrm{~mL})$, dried over $\mathrm{MgSO}_{4}$, filtered and concentrated under reduced pressure. Gradient flash chromatography (Petroleum ether/diethyl ether, 100:0 $\rightarrow$ 90:10 $\rightarrow$ 80:20) afforded the aldehyde $(1.30 \mathrm{~g}, 94 \%)$ as a clear pale yellow oil: $\mathrm{TLC} \mathrm{R}_{\mathrm{f}}=0.77\left(50 \% \mathrm{Et}_{2} \mathrm{O} / \mathrm{P} . \mathrm{E}.\right) ;[\alpha]_{D}^{25}$ +39.9 (c1.04, $\mathrm{CHCl}_{3}$ ); IR (neat) 2975 (s), 2936 (s), 2875 (s), 2164 (w), 1721 (s), 1639 (m), 1612 (s), 1586 (m), 1513 (s), 1457 (s), 1421 (m), 1394 (m), 1370 (m), 1346 (m), 1302 (s), 1246 (s), 1173 (s), 1033 (s), 1000 (s), 951 (s), 917 (s), 820 (s), 756 (s), 683 (s) $\mathrm{cm}^{-1}$; ${ }^{1} \mathrm{H} \mathrm{NMR}\left(400 \mathrm{MHz}, \mathrm{CDCl}_{3}\right) \delta 9.73\left(\mathrm{~d}, J=1.9 \mathrm{~Hz}, 1 \mathrm{H}, \mathrm{C}_{1}-H\right), 7.24(\mathrm{~d}, J=8.5 \mathrm{~Hz}$, $2 \mathrm{H}, \mathrm{Ar}-H_{2}, \mathrm{H}_{\mathrm{a}}$ ), 6.87 (d, $J=8.5 \mathrm{~Hz}, 2 \mathrm{H}, \mathrm{Ar}-H_{2}, \mathrm{H}_{\mathrm{b}}$ ), 5.77 (ddd, $J=17.7,10.7,7.9 \mathrm{~Hz}, 1$ $\left.\mathrm{H}, \mathrm{C}_{5}-H\right), 5.11\left(\mathrm{~d}, J=17.7 \mathrm{~Hz}, 1 \mathrm{H}, \mathrm{C}_{6}-H_{2}, \mathrm{H}_{\mathrm{c}}\right), 5.08\left(\mathrm{~d}, J=10.7 \mathrm{~Hz}, 1 \mathrm{H}, \mathrm{C}_{6}-H_{2}, \mathrm{H}_{\mathrm{d}}\right)$, $4.54\left(\mathrm{~d}, J=10.8 \mathrm{~Hz}, 1 \mathrm{H}\right.$, one of $\left.\mathrm{CH}_{2} \mathrm{Ar}\right), 4.48\left(\mathrm{~d}, J=10.8 \mathrm{~Hz}, 1 \mathrm{H}\right.$, one of $\left.\mathrm{CH}_{2} \mathrm{Ar}\right), 3.80$ $\left(\mathrm{s}, 3 \mathrm{H}, \mathrm{ArOCH}_{3}\right), 3.54\left(\mathrm{t}, J=6.1 \mathrm{~Hz}, 1 \mathrm{H}, \mathrm{C}_{3}-H\right), 2.69\left(\mathrm{~m}, 1 \mathrm{H}, \mathrm{C}_{2}-H\right), 2.54\left(\mathrm{~m}, 1 \mathrm{H}, \mathrm{C}_{4^{-}}\right.$ $H), 1.13\left(\mathrm{~d}, J=7.1 \mathrm{~Hz}, 3 \mathrm{H}, \mathrm{C}_{2} \mathrm{CH}_{3}\right), 1.11\left(\mathrm{~d}, J=6.8 \mathrm{~Hz}, 3 \mathrm{H}, \mathrm{C}_{4} \mathrm{CH}_{3}\right) ;{ }^{13} \mathrm{C} \mathrm{NMR}(100$ $\left.\mathrm{MHz}, \mathrm{CDCl}_{3}\right) \delta 204.4\left(\mathrm{C}_{1}\right), 159.2\left(\mathrm{Ar}, C-\mathrm{OCH}_{3}\right), 140.7\left(\mathrm{C}_{5}\right), 130.2(\mathrm{Ar}), 129.3(\mathrm{Ar}, C-$ $\left.\mathrm{H}_{\mathrm{a}}\right), 115.8\left(\mathrm{C}_{6}\right), 113.7\left(\mathrm{Ar}, \mathrm{C}-\mathrm{H}_{\mathrm{b}}\right), 84.2\left(\mathrm{C}_{3}\right), 73.4\left(\mathrm{CH}_{2} \mathrm{Ar}\right), 55.2\left(\mathrm{ArOCH}_{3}\right), 48.6\left(\mathrm{C}_{2}\right)$, $40.7\left(\mathrm{C}_{4}\right), 15.5\left(\mathrm{C}_{4} \mathrm{CH}_{3}\right), 11.1\left(\mathrm{C}_{2} \mathrm{CH}_{3}\right)$; high resolution mass spectrum $(+\mathrm{ESI}) \mathrm{m} / \mathrm{z}$ 285.1467 [(M+Na $)^{+}$; calcd for $\mathrm{C}_{16} \mathrm{H}_{22} \mathrm{O}_{3} \mathrm{Na}$ : 285.1467]; elemental analysis $\mathrm{C}, 72.97 \%$; $\mathrm{H}$, $8.45 \%$.

\section{1,1-Dibromo-3,5-dimethyl-4-(4'-Methoxy-benzyloxy)-hept-1,6-diene (12).}

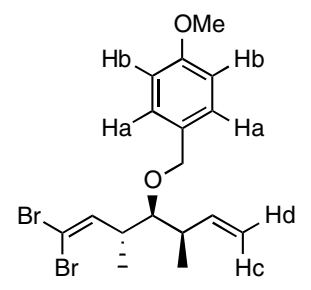

To a solution of triphenylphosphine $(3.99 \mathrm{~g}, 15.20 \mathrm{mmol})$ and carbon tetrabromide $(2.52$ $\mathrm{g}, 7.60 \mathrm{mmol})$ in dichloromethane $(50 \mathrm{~mL})$ stirred at $0{ }^{\circ} \mathrm{C}$ for $5 \mathrm{~min}$. was added a solution of 2(R), 3(S), 4(R), 3-(4-methoxy-benzyloxy)-2,4-dimethyl-hex-5-enal (1.00 g, 3.80 mmol) in dichloromethane $(10 \mathrm{~mL})$. The reaction mixture was stirred at $0{ }^{\circ} \mathrm{C}$ for $1 \mathrm{~h}$. The reaction mixture was quenched with saturated solution of aqueous $\mathrm{K}_{2} \mathrm{CO}_{3}$. The layers were separated, and the aqueous phase was extracted with dichloromethane $(3 \times 50$ $\mathrm{mL})$. The combined organic extracts were washed with $\mathrm{H}_{2} \mathrm{O}(1 \times 50 \mathrm{~mL})$, and brine $(1 \times$ $50 \mathrm{~mL}$ ), dried over $\mathrm{MgSO}_{4}$, filtered and concentrated under reduced pressure. Gradient flash chromatography (Petroleum ether/diethyl ether, 100:0 $\rightarrow$ 98:2 $\rightarrow$ 96:4 $\rightarrow$ 94:6) afforded $12(1.52 \mathrm{~g}, 87 \%)$ as a clear pale yellow oil: TLC $\mathrm{R}_{\mathrm{f}}=0.70$ (10\% $\mathrm{Et}_{2} \mathrm{O} / \mathrm{P} . \mathrm{E}$.); $[\alpha]_{D}^{25}-38.7$ (c 1.05, $\mathrm{CHCl}_{3}$ ); IR (neat) 2963 (s), 2930 (s), 2871 (s), 2835 (s), $2064(\mathrm{w})$, 1612 (s), 1586 (s), 1513 (s), 1455 (s), 1420 (m), 1396 (m), 1367 (m), 1349 (s), 1302 (s), 1247 (s), 1172 (s), 1036 (s), 995 (s), 956 (s), 916 (s), 821 (s), 789 (s), 757 (s), 682 (s) cm ${ }^{1} ;{ }^{1} \mathrm{H}$ NMR $\left(400 \mathrm{MHz}, \mathrm{CDCl}_{3}\right) \delta 7.28\left(\mathrm{~d}, J=8.6 \mathrm{~Hz}, 2 \mathrm{H}, \mathrm{Ar}-H_{2}, \mathrm{H}_{\mathrm{a}}\right), 6.90(\mathrm{~d}, J=8.6 \mathrm{~Hz}$, $\left.2 \mathrm{H}, \mathrm{Ar}-H_{2}, \mathrm{H}_{\mathrm{b}}\right), 6.51\left(\mathrm{~d}, J=9.5 \mathrm{~Hz}, 1 \mathrm{H}, \mathrm{C}_{2}-H\right), 5.72\left(\mathrm{ddd}, J=16.9,9.0,8.2 \mathrm{~Hz}, 1 \mathrm{H}, \mathrm{C}_{6}-\right.$ $H), 5.07\left(\mathrm{~d}, J=16.9 \mathrm{~Hz}, 1 \mathrm{H}, \mathrm{C}_{7}-H_{2}, \mathrm{H}_{\mathrm{c}}\right), 5.04\left(\mathrm{~d}, J=9.0 \mathrm{~Hz}, 1 \mathrm{H}, \mathrm{C}_{7}-H_{2}, \mathrm{H}_{\mathrm{d}}\right), 4.57(\mathrm{~d}, J$ $=10.7 \mathrm{~Hz}, 1 \mathrm{H}$, one of $\left.\mathrm{CH}_{2} \mathrm{Ar}\right), 4.50\left(\mathrm{~d}, J=10.7 \mathrm{~Hz}, 1 \mathrm{H}\right.$, one of $\left.\mathrm{CH}_{2} \mathrm{Ar}\right), 3.82(\mathrm{~s}, 3 \mathrm{H}$, 
$\left.\mathrm{ArOCH}_{3}\right), 3.14\left(\mathrm{dd}, J=7.9,2.8 \mathrm{~Hz}, 1 \mathrm{H}, \mathrm{C}_{4}-H\right), 2.74\left(\mathrm{~m}, 1 \mathrm{H}, \mathrm{C}_{3}-H\right), 2.36\left(\mathrm{~m}, 1 \mathrm{H}, \mathrm{C}_{5}-H\right)$, $1.10\left(\mathrm{~d}, J=6.2 \mathrm{~Hz}, 3 \mathrm{H}, \mathrm{C}_{3} \mathrm{CH}_{3}\right), 1.08\left(\mathrm{~d}, J=6.5 \mathrm{~Hz}, 3 \mathrm{H}, \mathrm{C}_{5} \mathrm{CH}_{3}\right) ;{ }^{13} \mathrm{C}$ NMR $(100 \mathrm{MHz}$, $\left.\mathrm{CDCl}_{3}\right) \delta 159.2\left(\mathrm{Ar}, C-\mathrm{OCH}_{3}\right), 140.6\left(\mathrm{C}_{2}\right), 140.6\left(\mathrm{C}_{6}\right), 130.6(\mathrm{Ar}), 129.4\left(\mathrm{Ar}-\mathrm{H}_{2}, C-\mathrm{H}_{\mathrm{a}}\right)$, $\left.115.1\left(\mathrm{C}_{7}\right), 113.7\left(\mathrm{Ar}-\mathrm{H}_{2}, C-\mathrm{H}_{\mathrm{b}}\right), 88.0\left(\mathrm{C}_{1}\right), 86.0\left(\mathrm{C}_{4}\right), 74.6\left(\mathrm{CH}_{2} \mathrm{Ar}\right), 55.3(\mathrm{ArOCH})_{3}\right), 42.1$ $\left(\mathrm{C}_{5}\right), 41.4\left(\mathrm{C}_{3}\right), 16.8\left(\mathrm{C}_{5} \mathrm{CH}_{3}\right), 16.6\left(\mathrm{C}_{3} \mathrm{CH}_{3}\right)$; high resolution mass spectrum $(+\mathrm{ESI}) \mathrm{m} / \mathrm{z}$ $438.9884\left[(\mathrm{M}+\mathrm{Na})^{+}\right.$; calcd for $\mathrm{C}_{17} \mathrm{H}_{22} \mathrm{O}_{2} \mathrm{Br}_{2} \mathrm{Na}$ : 438.9884]; elemental analysis $\mathrm{C}$, $49.00 \%$; $\mathrm{H}, 5.22 \%$.

5-(4'-Methoxy-benzyloxy)-4,6-dimethyl-oct-7-en-2-ynal (13).

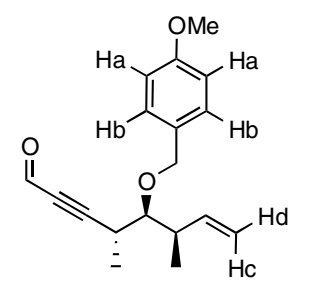

To a solution of 1,1-dibromo-3,5-dimethyl-4-(4'-Methoxy-benzyloxy)-hept-1,6-diene (12) $(0.80 \mathrm{~g}, 1.91 \mathrm{mmol})$ in tetrahydrofuran $(50 \mathrm{~mL})$ stirred at $-78{ }^{\circ} \mathrm{C}$ for $30 \mathrm{~min}$. was added a solution of $n \mathrm{BuLi}(1.6 \mathrm{M}$ in hexane) $(2.99 \mathrm{~mL}, 4.78 \mathrm{mmol})$. The reaction mixture was stirred at $-78{ }^{\circ} \mathrm{C}$ for $1 \mathrm{~h}$. To the reaction mixture was added a solution of $\mathrm{N}$ formylmorpholine $(0.31 \mathrm{~g}, 0.27 \mathrm{mmol})$ in tetrahydrofuran $(5 \mathrm{~mL})$. The reaction mixture was allowed to warm to ambient temperature over $3 \mathrm{~h}$. The reaction mixture was quenched with saturated solution of aqueous $\mathrm{NH}_{4} \mathrm{Cl}(50 \mathrm{~mL})$. The layers were separated, and the aqueous phase was extracted with diethyl ether $(3 \times 50 \mathrm{~mL})$. The combined organic extracts were washed with $\mathrm{H}_{2} \mathrm{O}(1 \times 50 \mathrm{~mL})$, and brine $(1 \times 50 \mathrm{~mL})$, dried over $\mathrm{MgSO}_{4}$, filtered and concentrated under reduced pressure. Gradient flash chromatography (Petroleum ether/diethyl ether, 100:0 $\rightarrow$ 95:5 $\rightarrow$ 90:10 $\rightarrow$ 88:12 $\rightarrow$ 84:16) afforded $13(0.40 \mathrm{~g}, 73 \%)$ as a clear pale yellow oil: TLC $\mathrm{R}_{\mathrm{f}}=0.27(10 \%$ $\mathrm{Et}_{2} \mathrm{O} /$ P.E.); $[\alpha]_{D}^{25}-19.5$ (c 1.025, $\mathrm{CHCl}_{3}$ ); IR (neat) 2976 (s), 2935 (s), 2875 (s), 2202 (s), 1662 (s), 1613 (s), 1586 (s), 1513 (s), 1457 (s), 1421 (s), 1389 (s), 1342 (s), 1302 (s), 1246 (s), 1172 (s), 1087 (s), 1062 (s), 1033 (s), 998 (s), 945 (s), 918 (s), 848 (s), 820 (s), $750(\mathrm{~s}), 682(\mathrm{~s}) \mathrm{cm}^{-1} ;{ }^{1} \mathrm{H} \mathrm{NMR}\left(400 \mathrm{MHz}, \mathrm{CDCl}_{3}\right) \delta 9.18\left(\mathrm{~s}, 1 \mathrm{H}, \mathrm{C}_{1}-H\right), 7.29(\mathrm{~d}, J=8.6$ $\left.\mathrm{Hz}, 2 \mathrm{H}, \mathrm{Ar}-H_{2}, \mathrm{H}_{\mathrm{a}}\right), 6.88\left(\mathrm{~d}, J=8.6 \mathrm{~Hz}, 2 \mathrm{H}, \mathrm{Ar}-H_{2}, \mathrm{H}_{\mathrm{b}}\right), 5.77$ (ddd, $J=17.1,10.2,8.4$ $\left.\mathrm{Hz}, 1 \mathrm{H}, \mathrm{C}_{7}-H\right), 5.15\left(\mathrm{~d}, J=17.1 \mathrm{~Hz}, 1 \mathrm{H}, \mathrm{C}_{8}-H_{2}, \mathrm{H}_{\mathrm{c}}\right), 5.05\left(\mathrm{dd}, J=10.2,0.8 \mathrm{~Hz}, 1 \mathrm{H}, \mathrm{C}_{8^{-}}\right.$ $\left.H_{2}, \mathrm{H}_{\mathrm{d}}\right), 4.59$ (s, $\left.2 \mathrm{H}, \mathrm{CH}_{2} \mathrm{Ar}\right), 3.81\left(\mathrm{~s}, 3 \mathrm{H}, \mathrm{ArOCH}_{3}\right), 3.21\left(\mathrm{dd}, J=7.4,4.4 \mathrm{~Hz}, 1 \mathrm{H}, \mathrm{C}_{5^{-}}\right.$ $H), 2.98\left(\mathrm{~m}, 1 \mathrm{H}, \mathrm{C}_{4}-H\right), 2.58\left(\mathrm{~m}, 1 \mathrm{H}, \mathrm{C}_{6}-H\right), 1.29\left(\mathrm{~d}, J=7.1 \mathrm{~Hz}, 3 \mathrm{H}, \mathrm{C}_{4} \mathrm{CH}_{3}\right), 1.11(\mathrm{~d}, J$ $\left.=6.7 \mathrm{~Hz}, 3 \mathrm{H}, \mathrm{C}_{6} \mathrm{CH}_{3}\right) ;{ }^{13} \mathrm{C} \mathrm{NMR}\left(100 \mathrm{MHz}, \mathrm{CDCl}_{3}\right) \delta 177.3\left(\mathrm{C}_{1}\right), 159.3\left(\mathrm{Ar}, \mathrm{C}-\mathrm{OCH}_{3}\right)$, $140.7\left(\mathrm{C}_{7}\right), 130.2(\mathrm{Ar}), 129.5\left(\mathrm{Ar}, C-\mathrm{H}_{\mathrm{b}}\right), 115.6\left(\mathrm{C}_{8}\right), 113.8\left(\mathrm{Ar}, C-\mathrm{H}_{\mathrm{a}}\right), 100.7\left(\mathrm{C}_{3}\right), 84.8$ $\left(\mathrm{C}_{5}\right), 83.0\left(\mathrm{C}_{2}\right), 74.7\left(\mathrm{CH}_{2} \mathrm{Ar}\right), 55.3\left(\mathrm{ArOCH}_{3}\right), 42.0\left(\mathrm{C}_{6}\right), 30.7\left(\mathrm{C}_{4}\right), 17.2\left(\mathrm{C}_{4} C H_{3}\right), 16.1$ $\left(\mathrm{C}_{6} \mathrm{CH}_{3}\right)$; high resolution mass spectrum $(+\mathrm{ESI}) \mathrm{m} / \mathrm{z} 309.1467\left[(\mathrm{M}+\mathrm{Na})^{+}\right.$; calcd for $\mathrm{C}_{18} \mathrm{H}_{22} \mathrm{O}_{3} \mathrm{Na}$ : 309.1467]; elemental analysis $\mathrm{C}, 75.46 \%$; $\mathrm{H}, 7.74 \%$.

7-(4'-Methoxy-benzyloxy)-6,8-dimethyl-dec-9-ene-1,4-diyn-3-ol 


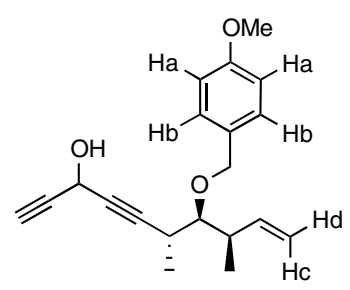

To a solution of 5-(4'-methoxy-benzyloxy)-4,6-dimethyl-oct-7-en-2-ynal (13) (262 mg, $0.91 \mathrm{mmol})$ in tetrahydrofuran $(10 \mathrm{~mL})$ stirred at $-78^{\circ} \mathrm{C}$ for $30 \mathrm{~min}$. was added a solution of ethynylmagnesium bromide $(0.5 \mathrm{M}$ in tetrahydrofuran) $(3.65 \mathrm{~mL}, 1.83 \mathrm{mmol})$. The reaction mixture was allowed to warm to $-30{ }^{\circ} \mathrm{C}$ and stirred for $30 \mathrm{~min}$. The reaction mixture was quenched with saturated solution of aqueous $\mathrm{NH}_{4} \mathrm{Cl}(10 \mathrm{~mL})$. The layers were separated, and the aqueous phase was extracted with diethyl ether $(3 \times 10 \mathrm{~mL})$. The combined organic extracts were washed with $\mathrm{H}_{2} \mathrm{O}(1 \times 10 \mathrm{~mL})$, and brine $(1 \times 10 \mathrm{~mL})$, dried over $\mathrm{MgSO}_{4}$, filtered and concentrated under reduced pressure. Gradient flash chromatography (Petroleum ether/diethyl ether, 100:0 $\rightarrow$ 90:10 $\rightarrow$ 80:20 $\rightarrow$ 70:30) afforded the bis-ynol $(230 \mathrm{mg}, 80 \%)$ as a clear pale yellow oil: TLC $\mathrm{R}_{\mathrm{f}}=0.08(10 \%$ Et $_{2}$ O/P.E.); IR (neat) 3290 (m, br), 2974 (m), 2934 (m), 2877 (m), 2238 (w), 2126 (w), 1637(m), 1612 (s), 1586 (m), 1513 (s), 1456 (s), 1420 (s), 1372 (s), 1345 (s), 1301 (s), 1246 (s), 1173 (s), 1093 (s), 1028 (s), 954 (s), 913 (s), 820 (s), 734 (s) cm ${ }^{-1} ;{ }^{1} \mathrm{H}$ NMR $\left(400 \mathrm{MHz}, \mathrm{CDCl}_{3}\right) \delta 7.31\left(\mathrm{~d}, J=8.6 \mathrm{~Hz}, 2 \mathrm{H}, \mathrm{Ar}-\mathrm{H}_{2}, \mathrm{H}_{\mathrm{a}}\right), 6.87(\mathrm{~d}, J=8.6 \mathrm{~Hz}, 2 \mathrm{H}, \mathrm{Ar}-$ $\mathrm{H}_{2}, \mathrm{H}_{\mathrm{b}}$ ), 5.81 (ddd, $\left.J=17.2,10.3,8.3 \mathrm{~Hz}, 1 \mathrm{H}, \mathrm{C}_{9}-H\right), 5.13\left(\mathrm{~d}, J=17.2 \mathrm{~Hz}, 1 \mathrm{H}, \mathrm{C}_{10}-H_{2}\right.$, $\mathrm{H}_{\mathrm{c}}$ ), $5.08\left(\mathrm{dd}, J=7.1,1.3 \mathrm{~Hz}, 1 \mathrm{H}, \mathrm{C}_{3}-H\right), 5.02\left(\mathrm{~d}, J=10.3 \mathrm{~Hz}, 1 \mathrm{H}, \mathrm{C}_{10}-H_{2}, \mathrm{H}_{\mathrm{d}}\right), 4.64(\mathrm{~d}$, $J=10.8 \mathrm{~Hz}, 1 \mathrm{H}$, one of $\left.\mathrm{CH}_{2} \mathrm{Ar}\right), 4.56\left(\mathrm{~d}, J=10.8 \mathrm{~Hz}, 1 \mathrm{H}\right.$, one of $\left.\mathrm{CH}_{2} \mathrm{Ar}\right), 3.80(\mathrm{~s}, 3 \mathrm{H}$, $\left.\mathrm{ArOCH}_{3}\right), 3.18\left(\mathrm{dd}, J=6.6,5.1 \mathrm{~Hz}, 1 \mathrm{H}, \mathrm{C}_{7}-H\right), 2.82\left(\mathrm{~m}, 1 \mathrm{H}, \mathrm{C}_{6}-H\right), 2.57\left(\mathrm{~m}, 1 \mathrm{H}, \mathrm{C}_{8}-H\right)$, $2.51\left(\mathrm{~d}, J=2.2 \mathrm{~Hz}, 1 \mathrm{H}, \mathrm{C}_{1}-H\right), 2.47\left(\mathrm{~d}, J=7.3 \mathrm{~Hz}, 1 \mathrm{H}, \mathrm{C}_{3} \mathrm{OH}\right), 1.23(\mathrm{~d}, J=7.1 \mathrm{~Hz}, 3 \mathrm{H}$, $\left.\mathrm{C}_{6} \mathrm{CH}_{3}\right), 1.09\left(\mathrm{~d}, J=6.8 \mathrm{~Hz}, 3 \mathrm{H}, \mathrm{C}_{8} \mathrm{CH}_{3}\right) ;{ }^{13} \mathrm{C} \mathrm{NMR}\left(100 \mathrm{MHz}, \mathrm{CDCl}_{3}\right) \delta 159.2(\mathrm{Ar}, \mathrm{C}-$ $\left.\mathrm{OCH}_{3}\right), 141.3\left(\mathrm{C}_{9}\right), 130.7$ (Ar), $130.7\left(\mathrm{Ar}{ }^{\prime}\right), 129.5\left(\mathrm{Ar}, C-\mathrm{H}_{\mathrm{a}}\right), 114.8\left(\mathrm{C}_{10}\right), 114.8\left(\mathrm{C}^{\prime}{ }_{10}\right)$, $113.7\left(\mathrm{Ar}, C-\mathrm{H}_{\mathrm{b}}\right), 87.6\left(\mathrm{C}_{5}\right), 87.6\left(C^{\prime}{ }_{5}\right), 85.3\left(\mathrm{C}_{7}\right), 81.5\left(\mathrm{C}_{3}\right), 81.5\left(\mathrm{C}^{\prime}{ }_{3}\right), 77.2\left(\mathrm{C}_{4}\right), 74.3$ $\left(\mathrm{CH}_{2} \mathrm{Ar}\right), 72.1\left(\mathrm{C}_{2}\right), 55.3\left(\mathrm{ArOCH}_{3}\right), 52.2\left(\mathrm{C}_{1}\right), 41.4\left(\mathrm{C}_{8}\right), 41.4\left(\mathrm{C}_{8}{ }_{8}\right), 29.9\left(\mathrm{C}_{6}\right), 17.6$ $\left(\mathrm{C}_{6} \mathrm{CH}_{3}\right), 17.6\left(\mathrm{C}_{6}{ }_{6} C^{\prime} \mathrm{H}_{3}\right), 15.7\left(\mathrm{C}_{8} C_{3}\right), 15.7\left(\mathrm{C}_{8}^{\prime} C^{\prime} \mathrm{H}_{3}\right)$; high resolution mass spectrum (+ESI) $m / z 335.1623\left[(\mathrm{M}+\mathrm{Na})^{+}\right.$; calcd for $\mathrm{C}_{20} \mathrm{H}_{24} \mathrm{O}_{3} \mathrm{Na}$ : 335.1623].

\section{7-(4'-Methoxy-benzyloxy)-6,8-dimethyl-dec-9-ene-1,4-diyn-3-one}

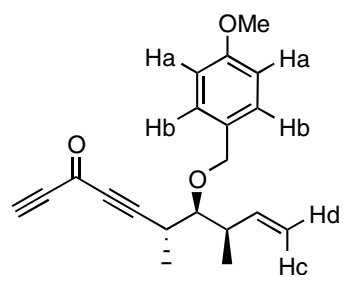

To a solution of 7-(4'-methoxy-benzyloxy)-6,8-dimethyl-dec-9-ene-1,4-diyn-3-ol (182 $\mathrm{mg}, 0.58 \mathrm{mmol})$ in dichloromethane $(5 \mathrm{~mL})$ stirred at $0{ }^{\circ} \mathrm{C}$ for $30 \mathrm{~min}$. was added DessMartin periodinane $(0.37 \mathrm{~g}, 0.88 \mathrm{mmol})$. The reaction mixture was allowed to warm to ambient temperature and stirred for $3 \mathrm{~h}$. The reaction mixture was quenched with a 1:1 solution of saturated aqueous $\mathrm{Na}_{2} \mathrm{~S}_{2} \mathrm{O}_{3}$ and saturated aqueous $\mathrm{NaHCO}_{3}(10 \mathrm{~mL})$. The 
layers were separated, and the aqueous phase was extracted with diethyl ether $(3 \times 10$ $\mathrm{mL})$. The combined organic extracts were washed with $\mathrm{H}_{2} \mathrm{O}(1 \times 10 \mathrm{~mL})$, and brine $(1 \times$ $10 \mathrm{~mL}$ ), dried over $\mathrm{MgSO}_{4}$, filtered and concentrated under reduced pressure. Gradient flash chromatography (Petroleum ether/diethyl ether, 100:0 $\rightarrow$ 90:10 $\rightarrow$ 80:20) afforded the bis-ynone $(157 \mathrm{mg}, 86 \%)$ as a clear yellow oil: $\mathrm{TLC}_{\mathrm{f}}=0.63\left(50 \% \mathrm{Et}_{2} \mathrm{O} / \mathrm{P} . \mathrm{E}\right.$.); $[\alpha]_{D}^{25}$ -18.2 (c 1.03, $\mathrm{CHCl}_{3}$ ); IR (neat) 3254 (m), 2977 (m), 2937 (m), 2876 (m), 2213 (s), 2097 (s), $1630(\mathrm{~s}), 1613(\mathrm{~s}), 1587(\mathrm{~m}), 1513(\mathrm{~s}), 1456(\mathrm{~s}), 1421(\mathrm{~m}), 1397(\mathrm{~m}), 1373(\mathrm{~m}), 1341$ (s), $1302(\mathrm{~s}), 1247(\mathrm{~s}), 1210(\mathrm{~s}), 1173(\mathrm{~s}), 1149(\mathrm{~s}), 1111(\mathrm{~s}), 1065(\mathrm{~s}), 1033(\mathrm{~s}), 954(\mathrm{~s})$, 918 (s), 821 (s), 727 (s), $682(\mathrm{~s}) \mathrm{cm}^{-1} ;{ }^{1} \mathrm{H}$ NMR $\left(400 \mathrm{MHz}, \mathrm{CDCl}_{3}\right) \delta 7.30(\mathrm{~d}, J=8.5 \mathrm{~Hz}$, $\left.2 \mathrm{H}, \mathrm{Ar}-\mathrm{H}_{2}, \mathrm{H}_{\mathrm{a}}\right), 6.88\left(\mathrm{~d}, J=8.5 \mathrm{~Hz}, 2 \mathrm{H}, \mathrm{Ar}-\mathrm{H}_{2}, \mathrm{H}_{\mathrm{b}}\right.$ ), 5.80 (ddd, $J=17.1,10.2,8.4 \mathrm{~Hz}, 1$ $\left.\mathrm{H}, \mathrm{C}_{9}-H\right), 5.15\left(\mathrm{~d}, J=17.1 \mathrm{~Hz}, 1 \mathrm{H}, \mathrm{C}_{10}-H_{2}, \mathrm{H}_{\mathrm{c}}\right), 5.06\left(\mathrm{~d}, J=10.2 \mathrm{~Hz}, 1 \mathrm{H}, \mathrm{C}_{10}-H_{2}, \mathrm{H}_{\mathrm{d}}\right)$, $4.62\left(\mathrm{~d}, J=10.7 \mathrm{~Hz}, 1 \mathrm{H}\right.$, one of $\left.\mathrm{CH}_{2} \mathrm{Ar}\right), 4.57\left(\mathrm{~d}, J=10.7 \mathrm{~Hz}, 1 \mathrm{H}\right.$, one of $\left.\mathrm{CH}_{2} \mathrm{Ar}\right), 3.81$ (s, $\left.3 \mathrm{H}, \mathrm{ArOCH}_{3}\right), 3.26\left(\mathrm{~m}, 1 \mathrm{H}, \mathrm{C}_{7}-H\right), 3.24\left(\mathrm{~s}, 1 \mathrm{H}, \mathrm{C}_{1}-H\right), 2.98\left(\mathrm{~m}, 1 \mathrm{H}, \mathrm{C}_{6}-H\right), 2.57(\mathrm{~m}$, $\left.1 \mathrm{H}, \mathrm{C}_{8}-H\right), 1.29\left(\mathrm{~d}, J=7.1 \mathrm{~Hz}, 3 \mathrm{H}, \mathrm{C}_{6} \mathrm{CH}_{3}\right), 1.10\left(\mathrm{~d}, J=6.7 \mathrm{~Hz}, 3 \mathrm{H}, \mathrm{C}_{8} \mathrm{CH}_{3}\right) ;{ }^{13} \mathrm{C}$ NMR $\left(100 \mathrm{MHz}, \mathrm{CDCl}_{3}\right) \delta 160.3\left(\mathrm{C}_{3}\right), 159.3\left(\mathrm{Ar}, C-\mathrm{OCH}_{3}\right), 140.7\left(\mathrm{C}_{9}\right), 130.3(\mathrm{Ar}), 129.4(\mathrm{Ar}$, $\left.C-\mathrm{H}_{\mathrm{b}}\right), 115.4\left(\mathrm{C}_{10}\right), 113.8\left(\mathrm{Ar}, C-\mathrm{H}_{\mathrm{a}}\right), 98.7\left(\mathrm{C}_{5}\right), 84.7\left(\mathrm{C}_{7}\right), 83.3\left(\mathrm{C}_{4}\right), 82.3\left(\mathrm{C}_{2}\right), 78.0\left(\mathrm{C}_{1}\right)$, $74.4\left(\mathrm{CH}_{2} \mathrm{Ar}\right), 55.3\left(\mathrm{ArOCH}_{3}\right), 41.6\left(\mathrm{C}_{8}\right), 30.6\left(\mathrm{C}_{6}\right), 16.7\left(\mathrm{C}_{6} \mathrm{CH}_{3}\right), 15.7\left(\mathrm{C}_{8} \mathrm{CH}_{3}\right)$; high resolution mass spectrum $(+\mathrm{ESI}) \mathrm{m} / \mathrm{z} 333.1467\left[(\mathrm{M}+\mathrm{Na})^{+}\right.$; calcd for $\mathrm{C}_{20} \mathrm{H}_{22} \mathrm{O}_{3} \mathrm{Na}$ : 333.1467].

\section{7-Hydroxy-6,8-dimethyl-dec-9-ene-1,4-diyn-3-one (14).}

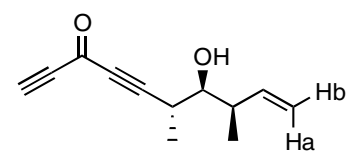

To a solution of 7-(4'-methoxy-benzyloxy)-6,8-dimethyl-dec-9-ene-1,4-diyn-3-one (50 $\mathrm{mg}, 0.16 \mathrm{mmol})$ in dichloromethane $(10 \mathrm{~mL})$ and $\mathrm{pH} 7$ buffer solution $(0.5 \mathrm{~mL})$ was added DDQ (55 mg, $0.24 \mathrm{mmol}$ ). The reaction mixture was stirred at ambient temperature for $1 \mathrm{~h}$ and $30 \mathrm{~min}$. TLC showed starting material to remain. Additional DDQ $(55 \mathrm{mg}, 0.24 \mathrm{mmol}$ ) was added and the reaction was stirred at ambient temperature for $14 \mathrm{~h}$. The reaction mixture was quenched with saturated aqueous $\mathrm{NaHCO}_{3}(10 \mathrm{~mL})$. The layers were separated, and the aqueous phase was extracted with diethyl ether $(3 \times$ $10 \mathrm{~mL})$. The combined organic extracts were washed with $\mathrm{H}_{2} \mathrm{O}(1 \times 10 \mathrm{~mL})$, and brine $(1$ $\times 10 \mathrm{~mL}$ ), dried over $\mathrm{MgSO}_{4}$, filtered and concentrated under reduced pressure. Gradient flash chromatography (Petroleum ether/diethyl ether, 100:0 $\rightarrow$ 90:10 $\rightarrow$ 80:20 $\rightarrow$ 70:30 $\rightarrow$ 60:40) afforded $14(31 \mathrm{mg}, 100 \%)$ as a clear pale yellow oil: $\mathrm{TLC} \mathrm{R}_{\mathrm{f}}=0.32(50 \%$ $\mathrm{Et}_{2} \mathrm{O} / \mathrm{P} . \mathrm{E}$.); $[\alpha]_{D}^{25}\left(c, \mathrm{CHCl}_{3}\right.$ ); IR (neat) 3450 (br, m), 3252 (br, m), $3080(\mathrm{w}), 2979(\mathrm{~m})$, 2937 (m), 2215 (s), 2097 (s), $1626(\mathrm{~s}), 1454(\mathrm{~m}), 1421(\mathrm{~m}), 1375(\mathrm{~m}), 1344(\mathrm{~m}), 1210(\mathrm{~s})$, $1137(\mathrm{~m}), 1058(\mathrm{~m}), 1036(\mathrm{~m}), 981(\mathrm{~s}), 913(\mathrm{~s}), 863(\mathrm{w}), 837(\mathrm{~m}), 726(\mathrm{~s}), 677(\mathrm{~s}) \mathrm{cm}^{-1}$; ${ }^{1} \mathrm{H}$ NMR $\left(600 \mathrm{MHz}, \mathrm{CDCl}_{3}\right) \delta 5.67$ (ddd, $\left.J=17.1,10.3,8.6 \mathrm{~Hz}, 1 \mathrm{H}, \mathrm{C}_{9}-H\right), 5.17(\mathrm{~d}, J=$ $\left.17.1 \mathrm{~Hz}, 1 \mathrm{H}, \mathrm{C}_{10}-H_{2}, \mathrm{H}_{\mathrm{a}}\right), 5.08\left(\mathrm{dd}, J=10.3,1.3 \mathrm{~Hz}, 1 \mathrm{H}, \mathrm{C}_{10}-H_{2}, \mathrm{H}_{\mathrm{b}}\right), 3.29\left(\mathrm{~m}, 1 \mathrm{H}, \mathrm{C}_{7^{-}}\right.$ $H), 2.95\left(\mathrm{~m}, 1 \mathrm{H}, \mathrm{C}_{6}-H\right), 2.41\left(\mathrm{~m}, 1 \mathrm{H}, \mathrm{C}_{8}-H\right), 1.66\left(\mathrm{~s}, 1 \mathrm{H}, \mathrm{C}_{1}-H\right), 1.33(\mathrm{~d}, J=7.1 \mathrm{~Hz}, 3$ $\left.\mathrm{H}, \mathrm{C}_{6}-\mathrm{CH}_{3}\right), 1.13\left(\mathrm{~d}, \mathrm{~J}=6.7 \mathrm{~Hz}, 3 \mathrm{H}, \mathrm{C}_{8}-\mathrm{CH}_{3}\right) ;{ }^{13} \mathrm{C} \mathrm{NMR}\left(150 \mathrm{MHz}, \mathrm{CDCl}_{3}\right) \delta 160.0$ $\left(\mathrm{C}_{3}\right), 139.9\left(\mathrm{C}_{9}\right), 116.3\left(\mathrm{C}_{10}\right), 96.7\left(\mathrm{C}_{2}\right), 84.0\left(\mathrm{C}_{4}\right), 82.2\left(\mathrm{C}_{5}\right), 78.4\left(\mathrm{C}_{1}\right), 77.4\left(\mathrm{C}_{7}\right), 43.3$ 
$\left(\mathrm{C}_{8}\right), 31.2\left(\mathrm{C}_{6}\right), 17.1\left(\mathrm{C}_{6}-\mathrm{CH}_{3}\right), 16.0\left(\mathrm{C}_{8}-\mathrm{CH}_{3}\right)$; high resolution mass spectrum (+ESI) $\mathrm{m} / \mathrm{z}$ $213.0891\left[(\mathrm{M}+\mathrm{Na})^{+}\right.$; calcd for $\mathrm{C}_{12} \mathrm{H}_{14} \mathrm{O}_{2} \mathrm{Na}$ : 213.0891].

\section{Dithiane (16).}

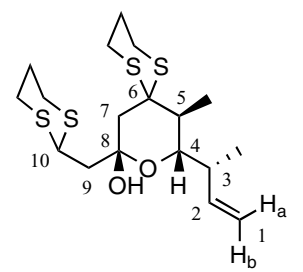

To a solution of 7-hydroxy-6,8-dimethyl-dec-9-ene-1,4-diyn-3-one (14) (59 mg, 0.31 $\mathrm{mmol})$ and propane-1,3-dithiol $(74 \mathrm{mg}, 0.68 \mathrm{mmol})$ in dichloromethane $(2 \mathrm{~mL})$ and methanol $(2 \mathrm{~mL})$ stirred at $-10{ }^{\circ} \mathrm{C}$ for $30 \mathrm{~min}$. was added sodium methoxide $(37 \mathrm{mg}, 0.68$ mmol). The reaction mixture was allowed to warm to ambient temperature over $24 \mathrm{~h}$. The reaction mixture was quenched with saturated aqueous $\mathrm{NH}_{4} \mathrm{Cl}$ solution $(5 \mathrm{~mL})$ and stirred for $10 \mathrm{~min}$. The layers were separated, and the aqueous phase was extracted with diethyl ether $(3 \times 10 \mathrm{~mL})$. The combined organic extracts were washed with $\mathrm{H}_{2} \mathrm{O}(1 \times 10$ $\mathrm{mL})$, and brine $(1 \times 10 \mathrm{~mL})$, dried over $\mathrm{MgSO}_{4}$, filtered and concentrated under reduced pressure. Gradient flash chromatography (Petroleum ether/diethyl ether, 100:0 $\rightarrow$ 90:10 $\rightarrow$ 80:20 $\rightarrow 70: 30 \rightarrow 60: 40)$ afforded $\mathbf{1 6}(82 \mathrm{mg}, 65 \%)$ as a white foam: TLC $\mathrm{R}_{\mathrm{f}}=0.49$ (50\% Et 2 O/P.E.); $[\alpha]_{D}^{25}+3.8$ (c 1.00, $\mathrm{CHCl}_{3}$ ); IR (neat) 3350 (br, m), $3075(\mathrm{w}), 2900(\mathrm{~m})$, 1638 (w), 1413 (s), $1382(\mathrm{~m}), 1341$ (m), 1275 (m), 1247 (m), 1163 (m), 1124 (s), 1073 (m), 1019 (s), 907 (s), $873(\mathrm{~m}), 840(\mathrm{~m}), 779(\mathrm{~m}), 752(\mathrm{~m}), 660(\mathrm{~m}) \mathrm{cm}^{-1} ;{ }^{1} \mathrm{H}$ NMR $(600$ $\mathrm{MHz}, \mathrm{CDCl}_{3}$ ) 6.02 (ddd, $\left.J=17.2,10.4,7.5 \mathrm{~Hz}, 1 \mathrm{H}, \mathrm{C}_{2}-H\right), 5.95\left(\mathrm{~s}, 1 \mathrm{H}, \mathrm{C}_{8}-\mathrm{OH}\right), 5.04(\mathrm{~d}$, $\left.J=17.2 \mathrm{~Hz}, 1 \mathrm{H}, \mathrm{C}_{1}-H_{2}, \mathrm{H}_{\mathrm{a}}\right), 4.98\left(\mathrm{~d}, J=10.4 \mathrm{~Hz}, \mathrm{C}_{1}-H_{2}, \mathrm{H}_{\mathrm{b}}\right), 4.40(\mathrm{t}, J=6.2 \mathrm{~Hz}, 1 \mathrm{H}$, $\left.\mathrm{C}_{10}-H\right), 3.77\left(\mathrm{dd}, J=10.4,2.1 \mathrm{~Hz}, 1 \mathrm{H}, \mathrm{C}_{4}-H\right), 3.16\left(\mathrm{dt}, J=14.7,2.4 \mathrm{~Hz}, 1 \mathrm{H}\right.$, one of $\mathrm{C}_{7^{-}}$ $\mathrm{H}_{2}$ ), 3.09 (app d, $J=14.6 \mathrm{~Hz}, 1 \mathrm{H}$, one of $\mathrm{SCH}_{2} \mathrm{CH}_{2} \mathrm{CH}_{2} \mathrm{~S}$ ), 2.93 (dt, $J=11.2,2.5 \mathrm{~Hz}, 2$ $\mathrm{H}$, two of $\left.\mathrm{SCH}_{2} \mathrm{CH}_{2} \mathrm{CH}_{2} \mathrm{~S}\right), 2.80\left(\mathrm{~m}, 3 \mathrm{H}\right.$, three of $\left.\mathrm{SCH}_{2} \mathrm{CH}_{2} \mathrm{CH}_{2} \mathrm{~S}\right), 2.68(\mathrm{~m}, 1 \mathrm{H}$, one of $\left.\mathrm{C}_{7}-\mathrm{H}_{2}\right), 2.40\left(\mathrm{t}, J=7.1 \mathrm{~Hz}, 1 \mathrm{H}, \mathrm{C}_{3}-\mathrm{H}\right), 2.14\left(\mathrm{~m}, 1 \mathrm{H}\right.$, two of $\left.\mathrm{SCH}_{2} \mathrm{CH}_{2} \mathrm{CH}_{2} \mathrm{~S}\right), 2.08(\mathrm{~m}, 2$ $\mathrm{H}$, two of $\mathrm{SCH}_{2} \mathrm{CH}_{2} \mathrm{CH}_{2} \mathrm{~S}$ ), 2.03 ( app dq, $\left.J=14.6,5.8 \mathrm{~Hz}, 2 \mathrm{H}, \mathrm{C}_{9}-\mathrm{H}_{2}\right), 1.84\left(\mathrm{~m}, 3 \mathrm{H}, \mathrm{C}_{5^{-}}\right.$ $H$ and two of $\left.\mathrm{SCH}_{2} \mathrm{CH}_{2} \mathrm{CH}_{2} \mathrm{~S}\right), 1.18\left(\mathrm{~d}, J=6.8 \mathrm{~Hz}, 3 \mathrm{H}, \mathrm{C}_{5} \mathrm{CH}_{3}\right), 0.99(\mathrm{~d}, J=7.0 \mathrm{~Hz}, 3 \mathrm{H}$, $\left.\mathrm{C}_{3} \mathrm{CH}_{3}\right) ;{ }^{13} \mathrm{C}$ NMR $\left(150 \mathrm{MHz}, \mathrm{CDCl}_{3}\right) \delta 142.5\left(\mathrm{C}_{2}\right), 113.5\left(\mathrm{C}_{1}\right), 95.9\left(\mathrm{C}_{8}\right), 72.1\left(\mathrm{C}_{4}\right), 53.4$ $\left(\mathrm{C}_{6}\right), 47.9\left(\mathrm{C}_{9}\right), 43.5\left(\mathrm{C}_{7}\right), 42.0\left(\mathrm{C}_{5}\right), 41.4\left(\mathrm{C}_{10}\right), 38.6\left(\mathrm{C}_{3}\right), 30.7\left(\mathrm{SCH}_{2} \mathrm{CH}_{2} \mathrm{CH}_{2} \mathrm{~S}\right), 30.7$ $\left(\mathrm{SCH}_{2} \mathrm{CH}_{2} \mathrm{CH}_{2} \mathrm{~S}\right), 25.8\left(\mathrm{SCH}_{2} \mathrm{CH}_{2} \mathrm{CH}_{2} \mathrm{~S}\right), 25.7\left(\mathrm{SCH}_{2} \mathrm{CH}_{2} \mathrm{CH}_{2} \mathrm{~S}\right), 25.5\left(\mathrm{SCH}_{2} \mathrm{CH}_{2} \mathrm{CH}_{2} \mathrm{~S}\right)$, $25.1\left(\mathrm{SCH}_{2} \mathrm{CH}_{2} \mathrm{CH}_{2} \mathrm{~S}\right), 11.9\left(\mathrm{C}_{3} \mathrm{CH}_{3}\right), 11.8\left(\mathrm{C}_{5} \mathrm{CH}_{3}\right)$; high resolution mass spectrum (+ESI) $\mathrm{m} / z$ 429.1026 [(M+Na) ${ }^{+}$; calcd for $\mathrm{C}_{18} \mathrm{H}_{30} \mathrm{O}_{2} \mathrm{~S}_{4} \mathrm{Na}$ : 429.1026]. 